\title{
Asymptotic Formulas for Macdonald Polynomials and the Boundary of the $(q, t)$-Gelfand-Tsetlin Graph
}

Cesar CUENCA

Department of Mathematics, Massachusetts Institute of Technology, USA

E-mail: cuenca@mit.edu

URL: http://math.mit.edu/〜 cuenca/

Received April 21, 2017, in final form December 09, 2017; Published online January 02, 2018

https://doi.org/10.3842/SIGMA.2018.001

\begin{abstract}
We introduce Macdonald characters and use algebraic properties of Macdonald polynomials to study them. As a result, we produce several formulas for Macdonald characters, which are generalizations of those obtained by Gorin and Panova in [Ann. Probab. 43 (2015), 3052-3132], and are expected to provide tools for the study of statistical mechanical models, representation theory and random matrices. As first application of our formulas, we characterize the boundary of the $(q, t)$-deformation of the Gelfand-Tsetlin graph when $t=q^{\theta}$ and $\theta$ is a positive integer.
\end{abstract}

Key words: Branching graph; Macdonald polynomials; Gelfand-Tsetlin graph

2010 Mathematics Subject Classification: 33D52; 33D90; 60B15; 60C05

\section{Introduction}

Macdonald polynomials are remarkable two-parameter $q, t$ generalizations of Schur polynomials. They were first introduced by Ian G. Macdonald in [31]; the canonical reference is his classical book [32]. The Macdonald polynomials are very interesting objects for representation theory and integrable systems, due to their connections with quantum groups, e.g., [20, 33], double affine Hecke algebras, e.g., [13, 28], etc. More recently, and releveant for us, Macdonald polynomials have been heavily used to study probabilistic models arising in mathematical physics and random matrix theory. The important work [4] of Borodin and Corwin showed how to use the algebraic properties of these polynomials to obtain analytic formulas that allow asymptotic analysis of the so-called Macdonald processes. Remarkably, by specialization or degeneration of the parameters $q, t$ defining Macdonald processes, the paper [4] yields tools that can be used to analyze interacting particle systems [5], beta Jacobi corners processes [6], probabilistic models from asymptotic representation theory [3], among others; see the survey [9] and references therein.

It should be noted that the special case $t=q$ of Macdonald processes are known as Schur processes and they have the special property of being determinantal point processes, thus allowing much more control over their asymptotics. Schur processes were introduced by Okounkov and Reshetikhin, as generalizations of the classical Plancherel measures, several years before the work of Borodin and Corwin [35,38]. The Schur processes, though a very special case of Macdonald processes, produced various applications to statistical models of plane partitions and random matrices, see for example [27, 39]. However, most of the physical models that were studied with the Macdonald processes do not have a determinantal structure, and therefore they could not have been analyzed solely by means of the Schur processes machinery.

The conclusion from the story of Schur and Macdonald processes is that studying the more complicated object can allow one to tackle more complicated questions, despite losing some integrability (such as the determinantal structure in the case of Schur processes). We follow this philosophy in our work, by introducing and studying Macdonald characters, two-parameter $q, t$ 
generalizations of normalized characters of unitary groups. The normalized characters of the unitary groups are expressible in terms of Schur polynomials, reason why we will call them Schur characters, whereas the generalization we present involves Macdonald polynomials.

Our main results are asymptotic formulas for Macdonald characters, which are generalizations of those for Schur characters, proved in [24] by different methods. As it is expected, the asymptotic formulas for Schur characters are simpler and they involve certain determinantal structure, whereas the formulas for Macdonald polynomials are more complicated and the determinantal structure is no longer present. However the advantage of our work on Macdonald polynomials, much like the advantage of Macdonald processes over Schur processes, is that we are able to access a number of asymptotic questions that are more general than those given in [24]. The tools we obtain in this paper are therefore very exciting, given that the formulas for Schur characters have already produced several applications to stochastic discrete particle systems, lozenge and domino tiling models, and asymptotic representation theory [10, 11, 12, 22, 24, 44].

The paper [15] is this article's companion, in which the author studies Jack characters, a natural degeneration of Macdonald characters and obtains their asymptotics in the VershikKerov limit regime. The approach to the study of asymptotics of Jack characters is different from the approach we use here to study the asymptotics of Macdonald characters; in particular, it relies heavily on the Pieri integral formula, see [15] for further details. The tools from this paper and [15] afford us a very strong control over the asymptotics of Macdonald characters, Jack characters and Bessel functions [36, 43], if the number of variables remains fixed and the rank tends to infinity. As another application of the developed toolbox, we have studied a Jack-Gibbs model of lozenge tilings in the spirit of $[7,25]$. The author was able to prove the weak convergence of statistics of the Jack-Gibbs lozenge tilings model near the edge of the boundary to the well-known Gaussian beta ensemble, see, e.g., Forrester [1, Chapter 20] and references therein. This result, and its rational limit concerning corner processes of Gaussian matrix ensembles, will appear in a forthcoming publication.

We proceed with a more detailed description of the results of the present paper.

\subsection{Description of the formulas}

The main object of study in this paper are the Macdonald characters, which we define as follows. For integers $1 \leq m \leq N$, a Macdonald character of rank $N$ and $m$ variables is a polynomial, with coefficients in $\mathbb{C}(q, t)$, of the form

$$
P_{\lambda}\left(x_{1}, \ldots, x_{m} ; N, q, t\right) \stackrel{\text { def }}{=} \frac{P_{\lambda}\left(x_{1}, \ldots, x_{m}, 1, t, \ldots, t^{N-m-1} ; q, t\right)}{P_{\lambda}\left(1, t, t^{2}, \ldots, t^{N-1} ; q, t\right)},
$$

where $P_{\lambda}\left(x_{1}, \ldots, x_{N} ; q, t\right)$ is the Macdonald polynomial of $N$ variables parametrized by the signature $\lambda=\left(\lambda_{1} \geq \lambda_{2} \geq \cdots \geq \lambda_{N}\right) \in \mathbb{Z}^{N}$. Macdonald characters, under the specialization $t=q$, turn into $q$-Schur characters, which have appeared previously in [21, 24]. The reason behind the use of the word "character" is that $q$-Schur characters turn into normalized characters of the irreducible rational representations of unitary groups, after the degeneration $q \rightarrow 1$.

We make one further comment about terminology. Macdonald characters, as defined here, are two-parameter $q, t$ degenerations of normalized and irreducible characters of unitary groups. One could also consider a two-parameter degeneration of the characters of the symmetric groups, in the spirit of Lassalle's work [29], where a one-parameter degeneration of symmetric group characters was considered. Thus a better name for our object would be Macdonald unitary character. For convenience, we simply will use the name Macdonald character. We remark that Macdonald symmetric group characters have not been considered yet, to the author's best knowledge. However, there have been many articles studying the structural theory and 
asymptotics on Jack symmetric group characters, notably several recent works by Maciej Dołęga, Valentin Féray and Piotr Śniady, see, e.g., [16, 17, 18, 46].

The main theorems of this paper fall into two categories:

(A) Integral representations for Macdonald characters of one variable and arbitrary rank $N$.

The initial idea that led to the integral representations in this paper is due to Andrei Okounkov, see [24, remark following Theorem 3.6]. An example of the integral formulas we prove is the following theorem. Observe that the integrand is a simple expression in terms of $q$-Gamma functions and can be analyzed by well known methods of asymptotic analysis, such as the method of steepest descent or the saddle-point method [14]. In this paper, we study the regime in which the signatures grow to infinity, whereas the remaining parameters are fixed.

Theorem 1.1 (consequence of Theorem 3.2). Assume $q \in(0,1)$ and $\theta>0$. Let $N \in \mathbb{N}, \lambda \in \mathbb{G T}_{N}$ and $x \in \mathbb{C} \backslash\{0\},|x| \leq q^{\theta(1-N)}$. The integral below converges absolutely and the identity holds

$$
\begin{aligned}
P_{\lambda}\left(x ; N, q, q^{\theta}\right)= & \frac{\ln q}{1-q} \frac{\left(x q^{\theta} ; q\right)_{\infty}}{\left(x q^{1+\theta(1-N)} ; q\right)_{\infty}} \frac{\Gamma_{q}(\theta N)}{2 \pi \sqrt{-1}} \\
& \times \int_{\mathcal{C}^{+}}\left(x q^{\theta(1-N)}\right)^{z} \prod_{i=1}^{N} \frac{\Gamma_{q}\left(\lambda_{i}+\theta(N-i)-z\right)}{\Gamma_{q}\left(\lambda_{i}+\theta(N-i+1)-z\right)} \mathrm{d} z,
\end{aligned}
$$

where $\mathcal{C}^{+}$is a certain contour described in Theorem 3.2, and which looks as in Fig. 2. In the formula above, we used the $q$-Pochhammer symbol $(z ; q)_{\infty}$ and the $q$-Gamma function $\Gamma_{q}(z)$; see Appendix A.

(B) Formulas expressing Macdonald characters of $m$ variables (and rank $N$ ) in terms of Macdonald characters of one variable (and $\operatorname{rank} N$ ).

These formulas will involve certain $q$-difference operators. Formulas of this kind will be called multiplicative formulas. ${ }^{1}$ One of the simplest multiplicative formulas we prove is the one below that expresses a Macdonald character of two variables in terms of those of one variable; the general formula is given below in Theorem 4.1.

Theorem 1.2 (reformulation of Corollary 4.2). Let $\theta \in \mathbb{N}, N \in \mathbb{N}, \lambda \in \mathbb{G T}_{N}$. Then

$$
\begin{aligned}
& P_{\lambda}\left(x_{1}, x_{2} ; N, q, q^{\theta}\right)=\frac{q^{-\left(N-\frac{3}{2}\right) \theta^{2}+\frac{1}{2} \theta}(1-q)^{\theta}}{\prod_{i=1}^{\theta}\left(1-q^{\theta N-i}\right)} \frac{1}{\prod_{i=1}^{\theta(N-1)-1}\left(x_{1}-q^{i-\theta}\right)\left(x_{2}-q^{i-\theta}\right)} \\
& \quad \times\left(\frac{1}{x_{1}-x_{2}} \circ\left(D_{q, x_{2}}-D_{q, x_{1}}\right)\right)^{\theta}\left\{\prod_{i=1}^{2}\left(P_{\lambda}\left(x_{i} ; N, q, q^{\theta}\right) \prod_{j=1}^{\theta N-1}\left(x_{i}-q^{j-\theta}\right)\right)\right\},
\end{aligned}
$$

where $D_{q, x_{i}}, i=1,2$, are the linear operators in $\mathbb{C}(q)\left[x_{1}, x_{2}\right]$ acting on monomials by $D_{q, x_{i}}\left(x_{1}^{m_{1}} x_{2}^{m_{2}}\right)$ $=\frac{1-q^{m_{i}}}{1-q}\left(x_{1}^{m_{1}} x_{2}^{m_{2}}\right), i=1,2$.

Observe that the multiplicative formula above requires $\theta \in \mathbb{N}$. It is somewhat surprising that all the identities we prove in this paper, even the integral representations, behave better for $\theta \in \mathbb{N}$.

\footnotetext{
${ }^{1}$ The reason for the name is that analogous formulas for Schur characters were used to prove statements of the form $\lim _{N \rightarrow \infty} F_{\lambda(N)}\left(x_{1}, \ldots, x_{m}\right)=\prod_{i=1}^{m} \lim _{N \rightarrow \infty} F_{\lambda(N)}\left(x_{i}\right)$, where the functions $F$ are certain normalizations of Schur characters, see, e.g., [24, Corollaries 3.10 and 3.12]
} 


\subsection{The boundary of the $(q, t)$-Gelfand-Tsetlin graph}

As an application of our formulas, we characterize the space of central probability measures in the path-space of the $(q, t)$-Gelfand-Tsetlin graph, when $t=q^{\theta}$ and $\theta$ is a positive integer. To state our result, we first introduce a few notions. Assume that $q, t \in(0,1)$ are generic real parameters for the moment.

The Gelfand-Tsetlin graph, or simply GT graph, is an undirected graph whose vertices are the signatures of all lengths $\mathbb{G} \mathbb{T}=\bigsqcup_{N \geq 0} \mathbb{G}_{N}$; we also include the empty signature $\varnothing$ as the only element of $\mathbb{G T}_{0}$ for convenience. The set of edges is determined by the interlacing constraints, namely the edges in the GT graph can only join signatures whose lengths differ by 1 and $\mu \in \mathbb{G T}_{N}$ is joined to $\lambda \in \mathbb{G T}_{N+1}$ if and only if

$$
\lambda_{N+1} \leq \mu_{N} \leq \lambda_{N} \leq \cdots \leq \lambda_{2} \leq \mu_{1} \leq \lambda_{1} .
$$

If the above inequalities are satisfied, we write $\mu \prec \lambda$. If $\mu \in \mathbb{G T}_{N}$ and $\lambda \in \mathbb{G T}_{N+1}$ are joined by an edge, then we consider the expression $\Lambda_{N}^{N+1}(\lambda, \mu)$ given by

$$
\Lambda_{N}^{N+1}(\lambda, \mu)=\psi_{\lambda / \mu}(q, t) \frac{P_{\mu}\left(t^{N}, \ldots, t^{2}, t ; q, t\right)}{P_{\lambda}\left(t^{N}, \ldots, t, 1 ; q, t\right)},
$$

where $\psi_{\lambda / \mu}(q, t)$ is given in the branching rule for Macdonald polynomials, see Theorem 2.5 below. If $\mu \in \mathbb{G T}_{N}$ is not joined to $\lambda \in \mathbb{G}_{N+1}$, set $\Lambda_{N}^{N+1}(\lambda, \mu)=0$. One can easily show, see, e.g., Theorem 2.3 below,

$$
\begin{aligned}
& \Lambda_{N}^{N+1}(\lambda, \mu) \geq 0, \quad \forall \lambda \in \mathbb{G T}_{N+1}, \quad \mu \in \mathbb{G}_{N}, \\
& \sum_{\mu \in \mathbb{G T}_{N}} \Lambda_{N}^{N+1}(\lambda, \mu)=1, \quad \forall \lambda \in \mathbb{G}^{N+1} .
\end{aligned}
$$

Thus, for any $N \in \mathbb{N}, \lambda \in \mathbb{G T}_{N+1}, \Lambda_{N}^{N+1}(\lambda, \cdot)$ is a probability measure on $\mathbb{G T}_{N}$. For this reason, we will call the expressions $\Lambda_{N}^{N+1}(\lambda, \mu)$ cotransition probabilities.

Next we define the path-space $\mathcal{T}$ of the GT graph as the set of infinite paths in the GT graph that begin at $\varnothing \in \mathbb{G T}_{0}: \mathcal{T}=\left\{\tau=\left(\varnothing=\tau^{(0)} \prec \tau^{(1)} \prec \tau^{(2)} \prec \cdots\right): \tau^{(n)} \in \mathbb{G}_{n} \forall n \in \mathbb{Z}_{\geq 0}\right\}$. Each finite path of the form $\phi=\left(\varnothing=\phi^{(0)} \prec \phi^{(1)} \prec \cdots \prec \phi^{(n)}\right)$ defines a cylinder set $S_{\phi}=\left\{\tau \in \mathcal{T}: \tau^{(1)}=\phi^{(1)}, \ldots, \tau^{(n)}=\phi^{(n)}\right\} \subset \mathcal{T}$. We equip $\mathcal{T}$ with the $\sigma$-algebra generated by the cylinder sets $S_{\phi}$, over all finite paths $\phi$. Equivalently, the $\sigma$-algebra of $\mathcal{T}$ is its Borel $\sigma$-algebra if we equip $\mathcal{T}$ with the topology it inherits as a subspace of the product $\prod_{n \geq 0} \mathbb{G T}_{n}$. Each probability measure $M$ on $\mathcal{T}$ admits a pushforward to a probability measure on $\mathbb{G T}_{m}$ via the obvious projection map

$$
\begin{aligned}
\operatorname{Proj}_{m}: \mathcal{T} & \subset \prod_{n \geq 0} \mathbb{G T}_{n} \longrightarrow \mathbb{G T}_{N}, \\
\tau & =\left(\tau^{(0)} \prec \tau^{(1)} \prec \tau^{(2)} \prec \cdots\right) \mapsto \tau^{(N)} .
\end{aligned}
$$

We say that a probability measure $M$ on $\mathcal{T}$ is a $(q, t)$-central measure if

$$
\begin{aligned}
& M\left(S\left(\phi^{(0)} \prec \phi^{(1)} \prec \cdots \prec \phi^{(N-1)} \prec \phi^{(N)}\right)\right) \\
& \quad=\Lambda_{N-1}^{N}\left(\phi^{(N)}, \phi^{(N-1)}\right) \cdots \Lambda_{0}^{1}\left(\phi^{(1)}, \phi^{(0)}\right) M_{N}\left(\phi^{(N)}\right),
\end{aligned}
$$

for all $N \geq 0$, all finite paths $\phi^{(0)} \prec \cdots \prec \phi^{(N)}$, and for some probability measures $M_{N}$ on $\mathbb{G T}_{N}$. It then automatically follows that $M_{N}=\left(\operatorname{Proj}_{N}\right)_{*} M$ are the pushforwards of $M$; moreover, they satisfy the coherence relations

$$
M_{N}(\mu)=\sum_{\lambda \in \mathbb{G T}_{N+1}} M_{N+1}(\lambda) \Lambda_{N}^{N+1}(\lambda, \mu), \quad \forall N \geq 0, \quad \forall \mu \in \mathbb{G}_{N}
$$


We denote by $M_{\text {prob }}(\mathcal{T})$ the set of $(q, t)$-central (probability) measures on $\mathcal{T}$; it is clearly a convex subset of the Banach space of all finite and signed measures on $\mathcal{T}$. Let us denote by $\Omega_{q, t}=\operatorname{Ex}\left(M_{\text {prob }}(\mathcal{T})\right)$ the set of its extreme points. From a general theorem, we can deduce that $\Omega_{q, t} \subset M_{\text {prob }}(\mathcal{T})$ is a Borel subset. We call $\Omega_{q, t}$, with its inherited topology, the boundary of the $(q, t)$-Gelfand-Tsetlin graph. The theorem stated below, which is our main application, completely characterizes the topological space $\Omega_{q, t}$. Before stating it, let us make a couple of relevant definitions.

Consider the set of weakly increasing integers $\mathcal{N}=\left\{\nu=\left(\nu_{1} \leq \nu_{2} \leq \cdots\right): \nu_{1}, \nu_{2}, \cdots \in \mathbb{Z}\right\}$ and equip it with the topology inherited from the product $\mathbb{Z}^{\infty}=\mathbb{Z} \times \mathbb{Z} \times \cdots$ of countably many discrete spaces. For each $k \in \mathbb{Z}$, we can define the automorphism $A_{k}$ of $\mathcal{N}$ by $\nu \mapsto A_{k} \nu=$ $\left(\nu_{1}+k \leq \nu_{2}+k \leq \cdots\right)$. Clearly $A_{k}$ has inverse $A_{-k}$. There is a similar automorphism of $\mathbb{G T}$, given by $\lambda \mapsto A_{k} \lambda=\left(\lambda_{1}+k \geq \lambda_{2}+k \geq \cdots\right), \varnothing \mapsto A_{k} \varnothing=\varnothing$, which restricts to automorphisms $\mathbb{G} \mathbb{T}_{m} \rightarrow \mathbb{G}_{m}$, for each $m \in \mathbb{Z}_{\geq 0}$.

For any $k \in \mathbb{Z}$, one can easily show that $\mu \in \mathbb{G}_{m}$ interlaces with $\lambda \in \mathbb{G T}_{m+1}$ iff $A_{k} \mu \in \mathbb{G}_{m}$ interlaces with $A_{k} \lambda \in \mathbb{G}_{m+1}$, that is, $\mu \prec \lambda$ iff $A_{k} \mu \prec A_{k} \lambda$. This allows us to define automorphisms $A_{k}$ of $\mathcal{T}$ by

$$
\begin{aligned}
A_{k}: & \mathcal{T} \longrightarrow \mathcal{T}, \\
& \tau=\left(\varnothing \prec \tau^{(1)} \prec \tau^{(2)} \prec \cdots\right) \mapsto A_{k} \tau=\left(\varnothing \prec A_{k} \tau^{(1)} \prec A_{k} \tau^{(2)} \prec \cdots\right) .
\end{aligned}
$$

One can similarly obtain maps $A_{k}$ on the set of finite paths of length $n$ by $\phi=\left(\phi^{(0)} \prec\right.$ $\left.\phi^{(1)} \prec \cdots \prec \phi^{(n)}\right) \mapsto A_{k} \phi=\left(A_{k} \phi^{(0)} \prec A_{k} \phi^{(1)} \prec \cdots \prec A_{k} \phi^{(n)}\right)$. Consequently we can also define automorphisms on cylinder sets by $A_{k} S_{\phi}=S_{A_{k} \phi}$, for all finite paths $\phi=\left(\phi^{(0)} \prec \phi^{(1)} \prec \cdots \prec \phi^{(n)}\right)$, in the natural way.

We named several maps above by the same letter $A_{k}$, but there should be no risk of confusion.

For our main theorem, we make the assumption $\theta \in \mathbb{N}, t=q^{\theta}$. We believe the theorem can be generalized for any $\theta>0$, but we do not have a proof at the moment.

Theorem 1.3. Assume $q \in(0,1), \theta \in \mathbb{N}$ and set $t=q^{\theta}$.

1. There exists a homeomorphism $\mathbf{N}: \mathcal{N} \rightarrow \Omega_{q, t}$ sending each $\nu \in \mathcal{N}$ to the $(q, t)$-central probability measure $M^{\nu} \in \Omega_{q, t}$ determined by the relations

$$
\begin{aligned}
& \sum_{\lambda \in \mathbb{G}_{m}} M_{m}^{\nu}(\lambda) \frac{P_{\lambda}\left(x_{1}, x_{2} t, \ldots, x_{m} t^{m-1} ; q, t\right)}{P_{\lambda}\left(1, t, \ldots, t^{m-1} ; q, t\right)}=\Phi^{\nu}\left(x_{1} t^{1-m}, \ldots, x_{m-1} t^{-1}, x_{m} ; q, t\right), \\
& \forall m \in \mathbb{N}, \quad \forall\left(x_{1}, \ldots, x_{m}\right) \in \mathbb{T}^{m} .
\end{aligned}
$$

In (1.2), we denoted by $\left\{M_{m}^{\nu}\right\}_{m \geq 1}$ the corresponding sequence of pushforwards of $M^{\nu}$ under the projection maps $\operatorname{Proj}_{m}: \mathcal{T} \rightarrow \mathbb{G}_{m}$. The left side in (1.2) is absolutely convergent on $\mathbb{T}^{m}, \mathbb{T}=\{z \in \mathbb{C}:|z|=1\}$, and the functions $\Phi^{\nu}$ in the right side are defined in (5.2) and (5.8). The probability measure $M^{\nu}$ is determined uniquely by the relations (1.2).

2. For each $k \in \mathbb{Z}$, the probability measures $M^{\nu}$ and $M^{A_{k} \nu}$ are related by

$$
M^{A_{k} \nu}\left(S_{A_{k} \phi}\right)=M^{\nu}\left(S_{\phi}\right), \quad \text { for all finite paths } \phi=\left(\phi^{(0)} \prec \phi^{(1)} \prec \cdots \prec \phi^{(n)}\right) .
$$

Moreover the $(q, t)$-coherent sequences $\left\{M_{m}^{\nu}\right\}_{m \geq 0}$ and $\left\{M_{m}^{A_{k} \nu}\right\}_{m \geq 0}$ are related by

$$
M_{m}^{A_{k} \nu}\left(A_{k} \lambda\right)=M_{m}^{\nu}(\lambda), \quad \forall m \geq 0, \quad \lambda \in \mathbb{G}_{m} .
$$

Another main result of this article is Theorem 7.9, where we characterize the Martin boundary of the $(q, t)$-Gelfand-Tseltin graph for $t=q^{\theta}$ and $\theta \in \mathbb{N}$. In fact, we first prove that the Martin boundary is homeomorphic to $\mathcal{N}$ and then show that the minimal boundary $\Omega_{q, t}$ coincides with the Martin boundary. See Sections 7.1 and 7.2 for the definition and characterization of the Martin boundary. 


\subsection{Comments on Theorem 1.3 and connections to existing literature}

Our first comment is that Theorem 1.3 is a generalization of the main theorem in the article of Vadim Gorin [21], which is the special case $\theta=1$ of our theorem, and characterizes the boundary of the $q$-Gelfand-Tsetlin graph. Some ideas in the proofs are the same, especially the overall scheme of using the ergodic method of Vershik-Kerov, see [47], but we need many new arguments as well. For example, [21] makes heavy use of the shifted Macdonald polynomials, in particular the binomial formula for shifted Macdonald polynomials at $t=q$, [34], while we do not use them at all. Moreover, in order to prove that the boundary of the $q$-Gelfand-Tsetlin graph is homeomorphic to $\mathcal{N}$, [21] made use of the following closed formula for the shifted-Schur generating function of $M_{N}^{\nu, \theta=1}$, in the case that $\nu_{1} \geq 0$ :

$$
\sum_{\lambda \in \mathbb{G T}_{N}^{+}} M_{N}^{\nu, \theta=1}(\lambda) \frac{s_{\lambda}^{*}\left(q^{N-1} x_{1}, \ldots, q^{N-1} x_{N} ; q^{-1}\right)}{s_{\lambda}^{*}\left(0, \ldots, 0 ; q^{-1}\right)}=H^{\nu}\left(x_{1}\right) \cdots H^{\nu}\left(x_{N}\right),
$$

where

$$
H^{\nu}(x)=\frac{\prod_{i=0}^{\infty}\left(1-q^{i} t\right)}{\prod_{j=1}^{\infty}\left(1-q^{\nu_{j}+j-1} t\right)}
$$

In the formula above, $s_{\lambda}^{*}\left(x_{1}, \ldots, x_{N} ; q\right)$ is the shifted Macdonald polynomial at $t=q$. In addition to the usefulness of the closed formula (1.5) above, the multiplicative structure is surprising. It would be interesting to find a closed formula for the shifted Macdonald generating function of the measures $M_{N}^{\nu}$, for general $\theta \in \mathbb{N}$, and find out if the multiplicative structure still holds in this generality.

It is shown in [21] that their main statement is equivalent to the characterization of certain Gibbs measures on lozenge tilings. A conjectural characterization of positive $q$-Toeplitz matrices is also given in that paper. Finally, it is mentioned that the asymptotics of $q$-Schur functions is related to quantum traces and the representation theory of $U_{\epsilon}\left(\mathfrak{g l}_{\infty}\right)$. It would be interesting to extend some of these statements to the Macdonald case, especially to connect the asymptotics of Macdonald characters to the representation theory of inductive limits of quantum groups.

Several other "boundary problems" have appeared in the literature in various contexts. For instance, in the limiting case $t=q \rightarrow 1$, the problem of characterizing the boundary $\Omega_{q, t}$ becomes equivalent to characterizing the space of extreme characters of the infinite-dimensional unitary group $\mathrm{U}(\infty)=\lim _{\rightarrow} \mathrm{U}(N)$. The answer also characterizes totally positive Toeplitz matrices $[19,47,48]$. Also in the degenerate case $t=q^{2}$ or $t=q^{1 / 2}$ and $q \rightarrow 1$, the boundary problem becomes equivalent to characterizing the space of extreme spherical functions of the infinitedimensional Gelfand pairs $(\mathrm{U}(2 \infty), \mathrm{Sp}(\infty))$ and $(\mathrm{U}(\infty), \mathrm{O}(\infty))$, respectively. This question, and in fact a more general one-parameter "Jack"-degeneration, was solved in [37]. A similar degenerate question in the setting of random matrix theory was studied in [42]. Some of the tools in this paper can be degenerated easily to these scenarios and they may provide an alternative approach to their proof as well; for example, the special case of our toolbox in the case $t=q \rightarrow 1$ was used in [24] to study the corresponding boundary problem, and in [15] we also study refine the asymptotic result that is needed to solve the boundary problem of [37].

Another similar boundary problem in a somewhat different direction is the following. Assume we consider the Young graph instead of the Gelfand-Tsetlin graph, e.g., see [8]. Assume also that the cotransition probabilities coming from the branching rule of Macdonald polynomials are replaced by the cotransition probabilities coming from the Pieri-rule. In this setting, the 
boundary problem has not been solved yet, but it is expected that the answer is given by Kerov's conjecture, which characterizes Macdonald-positive specializations, see [4, Section 2].

Finally, it was brought to my attention, after I completed the results of this paper, that Grigori Olshanski has obtained a characterization of the extreme set of $(q, t)$-central measures in the extended Gelfand-Tsetlin graph for more general parameters $q, t$ by different methods. His work follows the setting of the paper [23] of Gorin-Olshanski, which is some sort of analytic continuation to our proposed boundary problem. Interestingly, new features arise, e.g., two copies of the space $\mathcal{N}$ characterize the boundary in his context, one can define and work with suitable analogues of $z w$-measures, etc. Another related work in the $t=q$ case is his recent article [41].

\subsection{Organization of the paper}

The present work is organized as follows. In Section 2, we briefly recall some important algebraic properties of Macdonald polynomials that will be used to obtain our main results. We prove integral representations for Macdonald characters of one variable in Section 3. Next, in Section 4, we obtain multiplicative formulas for Macdonald characters of a given number of variables $m \in \mathbb{N}$ in terms of those of one variable. By making use of our formulas, in Section 5 we obtain asymptotics of Macdonald characters as the signatures grow to infinity in a specific limit regime. In Sections 6 and 7, we define and characterize the boundary of the $(q, t)$-Gelfand-Tsetlin graph in the case that $\theta \in \mathbb{N}$ and $t=q^{\theta}$. The asymptotic statements of Section 5 play the key role in the characterization of the boundary.

In Appendix A, we have bundled the necessary language and results of $q$-theory that are used throughout the paper. In Appendix B, we make some computations with expressions that appear in the multiplicative formulas for Macdonald polynomials.

\section{Symmetric Laurent polynomials}

A canonical reference for symmetric polynomials is [32]. We choose to give a brief overview of the tools that we need from [32], in order to fix terminology and to introduce lesser known objects, such as signatures and Macdonald Laurent polynomials.

\subsection{Partitions, signatures and symmetric Laurent polynomials}

A partition is a finite sequence of weakly decreasing nonnegative integers $\lambda=\left(\lambda_{1} \geq \lambda_{2} \geq \cdots \geq \lambda_{k}\right)$, $\lambda_{i} \in \mathbb{Z}_{\geq 0} \forall i$. We identify partitions that differ by trailing zeroes; for example, $(4,2,2,0,0)$ and $(4,2,2)$ are the same partition. We define the size of $\lambda$ to be the sum $|\lambda| \stackrel{\text { def }}{=} \lambda_{1}+\cdots+\lambda_{k}$, and its length $\ell(\lambda)$ to be the number of strictly positive elements of it. The dominance order for partitions is a partial order given by letting $\mu \leq \lambda$ if $|\mu|=|\lambda|$ and $\mu_{1}+\cdots+\mu_{i} \leq \lambda_{1}+\cdots+\lambda_{i}$ for all $i$. As usual, we let $\mu<\lambda$ if $\mu \leq \lambda$ and $\mu \neq \lambda$.

Partitions can be graphically represented by their Young diagrams. The Young diagram of partition $\lambda$ is the array of boxes with coordinates $(i, j)$ with $1 \leq j \leq \lambda_{i}, 1 \leq i \leq \ell(\lambda)$, where the coordinates are in matrix notation (row labels increase from top to bottom and column labels increase from left to right), see Fig. 1.

A signature is a sequence of weakly decreasing integers $\lambda=\left(\lambda_{1} \geq \lambda_{2} \geq \cdots \geq \lambda_{k}\right), \lambda_{i} \in \mathbb{Z} \forall i$. A positive signature is a signature whose elements are all nonnegative. The length of a signature, or positive signature, is the number $k$ of elements of it. Positive signatures which differ by trailing zeroes are not identified, in contrast to partitions; for example, $(4,2,2,0,0)$ and $(4,2,2)$ are different positive signatures, the first of length 5 and the second of length 3 . We shall denote $\mathbb{G}_{N}$ (resp. $\mathbb{G T}_{N}^{+}$) the set of signatures (resp. positive signatures) of length $N$. Evidently $\mathbb{G T}_{N}^{+}$can be identified with the set of all partitions of length $\leq N$. Under this identification, we are allowed 


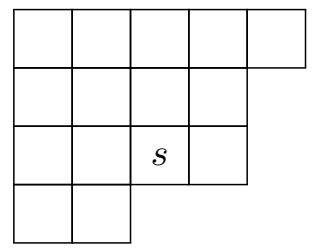

Figure 1. Young diagram for the partition $\lambda=(5,4,4,2)$. Square $s=(3,3)$ has arm length, arm colength, leg length and leg colength given by $a(s)=1, a^{\prime}(s)=2, l(s)=0, l^{\prime}(s)=2$.

to talk about the Young diagram of a positive signature $\lambda \in \mathbb{G} \mathbb{T}_{N}^{+}$, its size, the dominance order, and other attributes that are typically associated to partitions. Note, however, that length is defined differently for partitions and for positive signatures.

Let us now switch to notions pertaining to symmetric (Laurent) polynomials. Fix a positive integer $N$. Consider the field $F=\mathbb{C}(q, t)$ and recall the algebra $\Lambda_{F}\left[x_{1}, \ldots, x_{N}\right]$ of symmetric polynomials on the variables $x_{1}, \ldots, x_{N}$ with coefficients in $F$. For any $m \in \mathbb{Z}_{\geq 0}$, recall also the subalgebra $\Lambda_{F}^{m}\left[x_{1}, \ldots, x_{N}\right]$ of symmetric polynomials on $x_{1}, \ldots, x_{N}$ that are homogeneous of degree $m$; then

$$
\Lambda_{F}\left[x_{1}, \ldots, x_{N}\right]=\bigoplus_{m \geq 0} \Lambda_{F}^{m}\left[x_{1}, \ldots, x_{N}\right]
$$

We also denote by $\Lambda_{F}\left[x_{1}^{ \pm}, \ldots, x_{N}^{ \pm}\right]$the algebra of symmetric (with respect to the transpositions $x_{i} \leftrightarrow x_{i+1}$ for $i=1, \ldots, N-1$ ) Laurent polynomials in the variables $x_{1}, \ldots, x_{N}$.

The connection between partitions/signatures and symmetric polynomials comes from the observation that $\operatorname{dim}_{F}\left(\Lambda_{F}^{m}\left[x_{1}, \ldots, x_{N}\right]\right)$ is the number of partitions of size $m$ and length $\leq N$, or equivalently the number of positive signatures of size $m$ and length $N$. A basis for the space $\Lambda_{F}^{m}\left[x_{1}, \ldots, x_{N}\right]$ is given by the monomial symmetric polynomials $m_{\lambda}\left(x_{1}, \ldots, x_{N}\right)$, with $|\lambda|=m$, $\ell(\lambda) \leq N$, defined by

$$
m_{\lambda}\left(x_{1}, \ldots, x_{N}\right) \stackrel{\text { def }}{=} \sum_{\mu \in S_{N} \cdot \lambda} x_{1}^{\mu_{1}} \cdots x_{N}^{\mu_{N}},
$$

where $S_{N} \cdot \lambda$ is the orbit of $\lambda$ under the permutation action of $S_{N}$, and the sum runs over distinct elements $\mu$ of that orbit. It is implied that $\left\{m_{\lambda}\left(x_{1}, \ldots, x_{N}\right): \ell(\lambda) \leq N\right\}$ is a basis of $\Lambda_{F}\left[x_{1}, \ldots, x_{N}\right]$.

\subsection{Macdonald polynomials and Macdonald characters}

Proposition/Definition 2.1 ([32, Chapter VI, Sections 3, 4, 9]). The Macdonald polynomials $P_{\lambda}\left(x_{1}, \ldots, x_{N} ; q, t\right)$, for partitions $\lambda$ with $\ell(\lambda) \leq N$, are the unique elements of $\Lambda_{F}\left[x_{1}, \ldots, x_{N}\right]$ satisfying the following two properties

- Triangular decomposition: $P_{\lambda}\left(x_{1}, \ldots, x_{N} ; q, t\right)=m_{\lambda}+\sum_{\mu: \mu<\lambda} c_{\lambda, \mu} m_{\mu}$, for some $c_{\lambda, \mu} \in F$, and the sum is over partitions $\mu$ with $\ell(\mu) \leq N$, and $\mu<\lambda$ in the dominance order.

- Orthogonality relation: Let $[\cdot]_{0}: F\left[x_{1}^{ \pm}, \ldots, x_{N}^{ \pm}\right] \rightarrow F$ be the constant term map

$$
\left[\sum_{\lambda=\left(\lambda_{1} \geq \cdots \geq \lambda_{N}\right) \in \mathbb{Z}^{N}} a_{\lambda} x_{1}^{\lambda_{1}} \cdots x_{N}^{\lambda_{N}}\right]_{0}=a_{(0, \ldots, 0)} .
$$


The Macdonald polynomials are orthogonal with respect to the inner product $(\cdot, \cdot)_{q, t}$ on $\Lambda_{F}\left[x_{1}^{ \pm}, \ldots, x_{N}^{ \pm}\right]$given by $(f, g)_{q, t} \stackrel{\text { def }}{=}\left[f\left(x_{1}, \ldots, x_{N}\right) g\left(x_{1}^{-1}, \ldots, x_{N}^{-1}\right) \Delta_{q, t}\right]_{0}$, where

$$
\Delta_{q, t} \stackrel{\text { def }}{=} \prod_{1 \leq i \neq j \leq N} \prod_{k=0}^{\infty} \frac{1-q^{k} x_{i} x_{j}^{-1}}{1-q^{k} t x_{i} x_{j}^{-1}} .
$$

Note that $P_{\varnothing}(q, t)=1$. If $N<\ell(\lambda)$, we set $P_{\lambda}\left(x_{1}, \ldots, x_{N} ; q, t\right) \stackrel{\text { def }}{=} 0$ for convenience.

When we are talking about Macdonald polynomials and some of their properties which hold regardless of the number $N$ of variables, as long as $N$ is large enough, we simply write $P_{\lambda}(q, t)$ instead of $P_{\lambda}\left(x_{1}, \ldots, x_{N} ; q, t\right)$.

From the triangular decomposition of Macdonald polynomials, $P_{\lambda}(q, t)$ is a homogeneous polynomial of degree $|\lambda|$. Moreover $\left\{P_{\lambda}\left(x_{1}, \ldots, x_{N}\right): \ell(\lambda) \leq N\right\}$ is a basis of $\Lambda_{F}\left[x_{1}, \ldots, x_{N}\right]$. Finally, we have the following index stability property:

$$
P_{\left(\lambda_{1}+1, \ldots, \lambda_{N}+1\right)}\left(x_{1}, \ldots, x_{N} ; q, t\right)=\left(x_{1} \cdots x_{N}\right) \cdot P_{\lambda}\left(x_{1}, \ldots, x_{N} ; q, t\right) .
$$

As pointed out before, the set of partitions of length $\leq N$ is in bijection with $\mathbb{G T}_{N}^{+}$. Thus we can index the Macdonald polynomials by positive signatures rather than by partitions: for any $\lambda \in \mathbb{G T}_{N}^{+}$, we let $P_{\lambda}\left(x_{1}, \ldots, x_{N} ; q, t\right)$ be the Macdonald polynomial corresponding to the partition associated to $\lambda$. We can slightly extend the definition above and introduce Macdonald Laurent polynomials $P_{\lambda}\left(x_{1}, \ldots, x_{N} ; q, t\right)$ for any $\lambda \in \mathbb{G T}_{N}$. Let $\lambda \in \mathbb{G T}_{N}$ be arbitrary. If $\lambda_{N} \geq 0$, then $\lambda \in \mathbb{G T}_{N}^{+}$and $P_{\lambda}\left(x_{1}, \ldots, x_{N} ; q, t\right)$ is already defined. If $\lambda_{N}<0$, choose $m \in \mathbb{N}$ such that $\lambda_{N}+m \geq 0$ and so $\left(\lambda_{1}+m, \ldots, \lambda_{N}+m\right) \in \mathbb{G T}_{N}^{+}$. Then define

$$
P_{\lambda}\left(x_{1}, \ldots, x_{N} ; q, t\right) \stackrel{\text { def }}{=}\left(x_{1} \cdots x_{N}\right)^{-m} \cdot P_{\left(\lambda_{1}+m, \ldots, \lambda_{N}+m\right)}\left(x_{1}, \ldots, x_{N} ; q, t\right) .
$$

By virtue of the index stability property, the Macdonald (Laurent) polynomial $P_{\lambda}\left(x_{1}, \ldots, x_{N} ; q, t\right)$ is well-defined and does not depend on the value of $m$ that we choose. For simplicity, we call $P_{\lambda}\left(x_{1}, \ldots, x_{N} ; q, t\right)$ a Macdonald polynomial, whether $\lambda \in \mathbb{G T}_{N}^{+}$or not. In a similar fashion, we can define monomial symmetric polynomials $m_{\lambda}$, for any $\lambda \in \mathbb{G}_{N}$.

Recall the definitions of the arm-length, arm-colength, leg-length, leg-colength a $(s), a^{\prime}(s), l(s)$, $l^{\prime}(s)$ of the square $s=(i, j)$ of the Young diagram of $\lambda$, given by $a(s)=\lambda_{i}-j, a^{\prime}(s)=j-1$, $l(s)=\lambda_{j}^{\prime}-i, l^{\prime}(s)=i-1$; we note that $\lambda_{j}^{\prime}=\left|\left\{i: \lambda_{i} \geq j\right\}\right|$ is the length of the $j$ th part of the conjugate partition $\lambda^{\prime}$, see Fig. 1 .

We use terminology from $q$-analysis, see Appendix A; particularly we use the definition of $q$-Pochhammer symbols $(z ; q)_{n} \stackrel{\text { def }}{=} \prod_{i=0}^{n-1}\left(1-z q^{i}\right)$ and $(z ; q)_{\infty} \stackrel{\text { def }}{=} \prod_{i=0}^{\infty}\left(1-z q^{i}\right)$.

For $\lambda \in \bigsqcup_{N \geq 0} \mathbb{G} \mathbb{T}_{N}^{+}$, define the dual Macdonald polynomials $Q_{\lambda}(q, t)$ as the following normalization of Macdonald polynomials

$$
Q_{\lambda}(q, t) \stackrel{\text { def }}{=} b_{\lambda}(q, t) P_{\lambda}(q, t), \quad b_{\lambda}(q, t) \stackrel{\text { def }}{=} \prod_{s \in \lambda} \frac{1-q^{a(s)} t^{l(s)+1}}{1-q^{a(s)+1} t^{l(s)}} .
$$

The complete homogeneous symmetric (Macdonald) polynomials $g_{0}=1, g_{1}, g_{2}, \ldots$ are the one-row dual Macdonald polynomials:

$$
g_{n}(q, t) \stackrel{\text { def }}{=} Q_{(n)}(q, t)=\frac{(q ; q)_{n}}{(t ; q)_{n}} P_{(n)}(q, t) .
$$

For convenience, we also set $g_{n}(q, t) \stackrel{\text { def }}{=} 0, \forall n<0$.

Now we come to several important theorems on Macdonald polynomials, which will be our main tools. 
Theorem 2.2 (index-argument symmetry; [32, Chapter VI, Property 6.6]). Let $N \in \mathbb{N}, \lambda, \mu \in$ $\mathbb{G} \mathbb{T}_{N}^{+}$, then

$$
\frac{P_{\lambda}\left(q^{\mu_{1}} t^{N-1}, q^{\mu_{2}} t^{N-2}, \ldots, q^{\mu_{N}} ; q, t\right)}{P_{\lambda}\left(t^{N-1}, t^{N-2}, \ldots, 1 ; q, t\right)}=\frac{P_{\mu}\left(q^{\lambda_{1}} t^{N-1}, q^{\lambda_{2}} t^{N-2}, \ldots, q^{\lambda_{N}} ; q, t\right)}{P_{\mu}\left(t^{N-1}, t^{N-2}, \ldots, 1 ; q, t\right)} .
$$

Theorem 2.3 (evaluation identity; [32, Chapter VI, equations $(6.11)$ and $\left.\left(6.11^{\prime}\right)\right]$ ). Let $N \in \mathbb{N}$, $\lambda \in \mathbb{G} \mathbb{T}_{N}^{+}$, then

$$
\begin{aligned}
P_{\lambda}\left(t^{N-1}, t^{N-2}, \ldots, 1 ; q, t\right) & =t^{n(\lambda)} \prod_{1 \leq i<j \leq N} \frac{\left(q^{\lambda_{i}-\lambda_{j}} t^{j-i} ; q\right)_{\infty}\left(t^{j-i+1} ; q\right)_{\infty}}{\left(q^{\lambda_{i}-\lambda_{j}} t^{j-i+1} ; q\right)_{\infty}\left(t^{j-i} ; q\right)_{\infty}} \\
& =t^{n(\lambda)} \prod_{s \in \lambda} \frac{1-q^{a^{\prime}(s)} t^{N-l^{\prime}(s)}}{1-q^{a(s)} t^{l(s)+1}}
\end{aligned}
$$

where and $n(\lambda) \stackrel{\text { def }}{=} \lambda_{2}+2 \lambda_{3}+\cdots+(N-1) \lambda_{N}$. The first equality holds, more generally, for any signature $\lambda \in \mathbb{G T}_{N}$ by virtue of the definition (2.2) of Macdonald Laurent polynomials $P_{\lambda}\left(x_{1}, \ldots, x_{N} ; q, t\right)$.

From the second equality of Theorem 2.3 and the definition of dual Macdonald polynomials, we obtain

Corollary 2.4. Let $N \in \mathbb{N}, \lambda \in \mathbb{G T}_{N}^{+}$, then

$$
Q_{\lambda}\left(t^{N-1}, t^{N-2}, \ldots, 1 ; q, t\right)=t^{n(\lambda)} \prod_{s \in \lambda} \frac{1-q^{a^{\prime}(s)} t^{N-l^{\prime}(s)}}{1-q^{a(s)+1} t^{l(s)}} .
$$

Since the Macdonald polynomial $P_{\lambda}\left(x_{1}, x_{2}, \ldots, x_{N} ; q, t\right)$ is symmetric in $x_{1}, x_{2}, \ldots, x_{N}$, it is also a symmetric polynomial on $x_{2}, \ldots, x_{N}$; thus it is a linear combination of Macdonald polynomials $P_{\mu}\left(x_{2}, \ldots, x_{N} ; q, t\right)$ with coefficients in $F\left[x_{1}\right]$. More precisely, we have the so-called branching rule for Macdonald polynomials:

Theorem 2.5 (branching rule; [32, Chapter VI, equation (7.13'), Example 2(b) on p. 342]). Let $N \in \mathbb{N}, \lambda \in \mathbb{G} \mathbb{T}_{N}^{+}$, then

$$
P_{\lambda}\left(x_{1}, x_{2}, \ldots, x_{N} ; q, t\right)=\sum_{\mu \in \mathbb{G T}_{N-1}^{+}: \mu \prec \lambda} \psi_{\lambda / \mu}(q, t) x_{1}^{|\lambda|-|\mu|} P_{\mu}\left(x_{2}, \ldots, x_{N} ; q, t\right),
$$

where the branching coefficients are

$$
\begin{aligned}
\psi_{\lambda / \mu}(q, t) \stackrel{\text { def }}{=} \prod_{1 \leq i \leq j \leq N-1} \frac{\left(q^{\mu_{i}-\mu_{j}} t^{j-i+1} ; q\right)_{\infty}\left(q^{\lambda_{i}-\lambda_{j+1}} t^{j-i+1} ; q\right)_{\infty}}{\left(q^{\lambda_{i}-\mu_{j}} t^{j-i+1} ; q\right)_{\infty}\left(q^{\mu_{i}-\lambda_{j+1}} t^{j-i+1} ; q\right)_{\infty}} \\
\times \frac{\left(q^{\lambda_{i}-\mu_{j}+1} t^{j-i} ; q\right)_{\infty}\left(q^{\mu_{i}-\lambda_{j+1}+1} t^{j-i} ; q\right)_{\infty}}{\left(q^{\mu_{i}-\mu_{j}+1} t^{j-i} ; q\right)_{\infty}\left(q^{\lambda_{i}-\lambda_{j+1}+1} t^{j-i} ; q\right)_{\infty}},
\end{aligned}
$$

and the sum is over positive signatures $\mu \in \mathbb{G T}_{N-1}^{+}$that satisfy the interlacing constraint

$$
\lambda_{N} \leq \mu_{N-1} \leq \lambda_{N-1} \leq \cdots \leq \mu_{1} \leq \lambda_{1}
$$

which is written succinctly as $\mu \prec \lambda$.

Observe that $\psi_{\lambda / \mu}(q, t)>0$, whenever $q, t \in(0,1)$. By applying the branching rule several times, we can deduce the following. 
Corollary 2.6. The coefficients $c_{\lambda, \mu}=c_{\lambda, \mu}(q, t)$ in the expansion

$$
P_{\lambda}(q, t)=\sum_{\mu} c_{\lambda, \mu} m_{\mu}
$$

are such that $c_{\lambda, \lambda}=1$ and $c_{\lambda, \mu} \geq 0$, whenever $q, t \in(0,1)$. Moreover, $c_{\lambda, \mu}=0$ unless $\lambda \geq \mu$.

We come to our final tool on Macdonald polynomials. It is the main theorem of [30], and is called the Jacobi-Trudi formula for Macdonald polynomials. For any $n \in \mathbb{N}$, nonnegative integers $\tau_{1}, \ldots, \tau_{n}$, variables $u_{1}, \ldots, u_{n}$, define the rational functions $C_{\tau_{1}, \ldots, \tau_{n}}^{(q, t)}\left(u_{1}, \ldots, u_{n}\right)$ by

$$
\begin{aligned}
& C_{\tau_{1}, \ldots, \tau_{n}}^{(q, t)}\left(u_{1}, \ldots, u_{n}\right) \stackrel{\text { def }}{=} \prod_{k=1}^{n} t^{\tau_{k}} \frac{(q / t ; q)_{\tau_{k}}}{(q ; q)_{\tau_{k}}} \frac{\left(q u_{k} ; q\right)_{\tau_{k}}}{\left(q t u_{k} ; q\right)_{\tau_{k}}} \prod_{1 \leq i<j \leq n} \frac{\left(q u_{i} / t u_{j} ; q\right)_{\tau_{i}}}{\left(q u_{i} / u_{j} ; q\right)_{\tau_{i}}} \frac{\left(t u_{i} /\left(q^{\tau_{i}} u_{j}\right) ; q\right)_{\tau_{i}}}{\left(u_{i} /\left(q^{\tau_{i}} u_{j}\right) ; q\right)_{\tau_{i}}} \\
& \quad \times \frac{1}{\Delta\left(q^{\tau_{1}} u_{1}, \ldots, q^{\tau_{n}} u_{n}\right)} \operatorname{det}_{1 \leq i, j \leq n}\left[\left(q^{\tau_{i}} u_{i}\right)^{n-j}\left(1-t^{j-1} \frac{1-t q^{\tau_{i}} u_{i}}{1-q^{\tau_{i}} u_{i}} \prod_{k=1}^{n} \frac{u_{k}-q^{\tau_{i}} u_{i}}{t u_{k}-q^{\tau_{i}} u_{i}}\right)\right],(2.5)
\end{aligned}
$$

where $\Delta\left(z_{1}, \ldots, z_{n}\right) \stackrel{\text { def }}{=} \prod_{1 \leq i<j \leq n}\left(z_{i}-z_{j}\right)=\operatorname{det}\left[z_{i}^{n-j}\right]_{i, j=1}^{n}$ is known as the Vandermonde determinant.

Theorem 2.7 (Jacobi-Trudi formula; [30, Theorem 5.1]). Let $N \in \mathbb{N}, \lambda \in \mathbb{G T}_{N}^{+}$, then

$$
\begin{aligned}
Q_{\lambda}\left(x_{1}, \ldots, x_{N} ; q, t\right) & \\
= & \sum_{\tau \in M^{(N)}}\left\{\prod_{s=1}^{N-1} C_{\tau_{1, s+1}, \ldots, \tau_{s, s+1}}^{(q, t)}\left(u_{i}=q^{\lambda_{i}-\lambda_{s+1}+\sum_{j=s+2}^{N}\left(\tau_{i, j}-\tau_{s+1, j}\right)} t^{s-i}: 1 \leq i \leq s\right)\right. \\
& \left.\times \prod_{s=1}^{N} g_{\lambda_{s}+\tau_{s}^{+}-\tau_{s}^{-}}\left(x_{1}, \ldots, x_{N} ; q, t\right)\right\},
\end{aligned}
$$

where $M^{(N)}$ is the set of strictly upper-triangular matrices with nonnegative entries, and for each $1 \leq s \leq N$, the integers $\tau_{s}^{+}, \tau_{s}^{-}$, depend only on the indexing matrix $\tau$ and are defined by

$$
\tau_{s}^{+} \stackrel{\text { def }}{=} \sum_{i=s+1}^{N} \tau_{s, i}, \quad \tau_{s}^{-} \stackrel{\text { def }}{=} \sum_{i=1}^{s-1} \tau_{i, s}
$$

Remark 2.8. Observe that, even though $M^{(N)}$ is an infinite set, the only nonvanishing terms in the sum above are those $\tau \in M^{(N)}$ such that $\lambda_{s}+\tau_{s}^{+}-\tau_{s}^{-} \geq 0 \forall s=1, \ldots, N$. In other words, the sum is indexed by points of the discrete $\frac{N(N-1)}{2}$-dimensional simplex with coordinates $\left\{\tau_{i, j}\right\}_{1 \leq i<j \leq N}$ satisfying

$$
\begin{aligned}
& \tau_{i, j} \geq 0, \quad \text { for all } 1 \leq i<j \leq N \\
& \lambda_{n}+\sum_{i=n+1}^{N} \tau_{n, i}-\sum_{i=1}^{n-1} \tau_{i, n} \geq 0, \quad \text { for all } n=1, \ldots, N .
\end{aligned}
$$

Let us introduce the last piece of terminology and main object of study in this paper.

Definition 2.9. For any $m, N \in \mathbb{N}$ with $1 \leq m \leq N, \lambda \in \mathbb{G T}_{N}$, define

$$
P_{\lambda}\left(x_{1}, \ldots, x_{m} ; N, q, t\right) \stackrel{\text { def }}{=} \frac{P_{\lambda}\left(x_{1}, \ldots, x_{m}, 1, t, \ldots, t^{N-m-1} ; q, t\right)}{P_{\lambda}\left(1, t, t^{2}, \ldots, t^{N-1} ; q, t\right)}
$$

and call $P_{\lambda}\left(x_{1}, \ldots, x_{m} ; N, q, t\right)$ the Macdonald unitary character of rank $N$, number of variables $m$ and parametrized by $\lambda$. For simplicity of terminology, we call $P_{\lambda}\left(x_{1}, \ldots, x_{m} ; N, q, t\right)$ a Macdonald character rather than a Macdonald unitary character. Observe that if $q, t \in \mathbb{C}$ are such that $|q|,|t| \in(0,1)$, the evaluation identity for Macdonald polynomials, Theorem 2.3, shows that the denominator of (2.7) is nonzero. 


\section{Integral formulas for Macdonald characters of one variable}

In this section, assume $q$ is a real number in the interval $(0,1)$. There will also be a parameter $\theta$, typically $\theta>0$, but we also consider cases when $\theta$ is a complex number with $\Re \theta>0$. In either case, the parameter $t=q^{\theta}$ satisfies $|t|<1$.

\subsection{Statements of the theorems}

The simplest contour integral representation is the following, which works only when $t=q^{\theta}$, $\theta \in \mathbb{N}$, and involves a closed contour around finitely many singularities.

Theorem 3.1. Let $\theta \in \mathbb{N}, t=q^{\theta}, N \in \mathbb{N}, \lambda \in \mathbb{G}_{N}$ and $x \in \mathbb{C} \backslash\left\{0, q, q^{2}, \ldots, q^{\theta N-1}\right\}$. Then

$$
\begin{aligned}
& \frac{P_{\lambda}\left(x, t, t^{2}, \ldots, t^{N-1} ; q, t\right)}{P_{\lambda}\left(1, t, t^{2}, \ldots, t^{N-1} ; q, t\right)} \\
& \quad=\ln (1 / q) \prod_{i=1}^{\theta N-1} \frac{1-q^{i}}{x-q^{i}} \frac{1}{2 \pi \sqrt{-1}} \oint_{\mathcal{C}_{0}} \frac{x^{z}}{\prod_{i=1}^{N} \prod_{j=0}^{\theta-1}\left(1-q^{z-\left(\lambda_{i}+\theta(N-i)+j\right)}\right)} \mathrm{d} z,
\end{aligned}
$$

where $\mathcal{C}_{0}$ is a closed, positively oriented contour enclosing the real poles $\left\{\lambda_{i}+\theta(N-i)+j: i=\right.$ $1, \ldots, N, j=0, \ldots, \theta-1\}$ of the integrand. For instance, the rectangular contour with vertices $-M-r \sqrt{-1},-M+r \sqrt{-1}, M+r \sqrt{-1}$ and $M-r \sqrt{-1}$, for any $-\frac{2 \pi}{\ln q}>r>0$ and any $M>\max \left\{0,-\lambda_{N}, \lambda_{1}+\theta N-1\right\}$, is a suitable contour.

The following two theorems are analytic continuations, in the variable $\theta$, of Theorem 3.1 above.

Theorem 3.2. Let $\theta>0, t=q^{\theta}, N \in \mathbb{N}, \lambda \in \mathbb{G T}_{N}$ and $x \in \mathbb{C} \backslash\{0\},|x| \leq 1$. The integral below converges absolutely and the equality holds

$$
\begin{aligned}
& \frac{P_{\lambda}\left(x t^{N-1}, t^{N-2}, \ldots, t, 1 ; q, t\right)}{P_{\lambda}\left(t^{N-1}, t^{N-2}, \ldots, t, 1 ; q, t\right)} \\
& \quad=\frac{\ln q}{1-q} \frac{\left(x t^{N} ; q\right)_{\infty}}{(x q ; q)_{\infty}} \frac{\Gamma_{q}(\theta N)}{2 \pi \sqrt{-1}} \int_{\mathcal{C}^{+}} x^{z} \prod_{i=1}^{N} \frac{\Gamma_{q}\left(\lambda_{i}+\theta(N-i)-z\right)}{\Gamma_{q}\left(\lambda_{i}+\theta(N-i+1)-z\right)} \mathrm{d} z .
\end{aligned}
$$

Contour $\mathcal{C}^{+}$is a positively oriented contour consisting of the segment $[M+r \sqrt{-1}, M-r \sqrt{-1}]$ and the horizontal lines $[M+r \sqrt{-1},+\infty+r \sqrt{-1}),[M-r \sqrt{-1},+\infty-r \sqrt{-1})$, for some $-\frac{\pi}{2 \ln q}>r>0$ and $\lambda_{N}>M$, see Fig. 2. Observe that $\mathcal{C}^{+}$encloses all real poles of the integrand (which accumulate at $+\infty)$ and no other poles.

The reader is referred to Appendix A for a reminder of the definition of the $q$-Gamma function, its zeroes and poles.

Theorem 3.3. Let $\theta>0, t=q^{\theta}, N \in \mathbb{N}, \lambda \in \mathbb{G T}_{N}$ and $x \in \mathbb{C},|x| \geq 1$. The integral below converges absolutely and the equality holds

$$
\begin{aligned}
& \frac{P_{\lambda}\left(x, t, t^{2}, \ldots, t^{N-1} ; q, t\right)}{P_{\lambda}\left(1, t, t^{2}, \ldots, t^{N-1} ; q, t\right)} \\
& \quad=\frac{\ln q}{q-1} \frac{\left(x^{-1} t^{N} ; q\right)_{\infty}}{\left(x^{-1} q ; q\right)_{\infty}} \frac{\Gamma_{q}(\theta N)}{2 \pi \sqrt{-1}} \int_{\mathcal{C}^{-}} x^{z} \prod_{i=1}^{N} \frac{\Gamma_{q}\left(z-\left(\lambda_{i}-\theta i+\theta\right)\right)}{\Gamma_{q}\left(z-\left(\lambda_{i}-\theta i\right)\right)} \mathrm{d} z .
\end{aligned}
$$




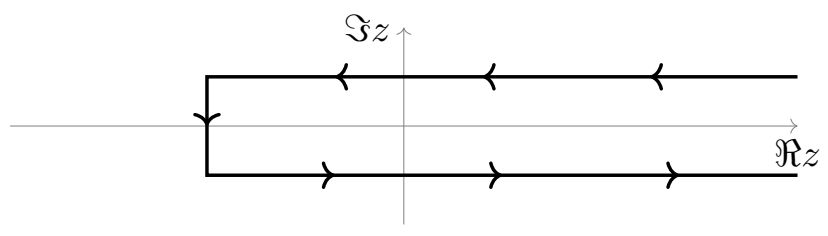

Figure 2. Contour $\mathcal{C}^{+}$.

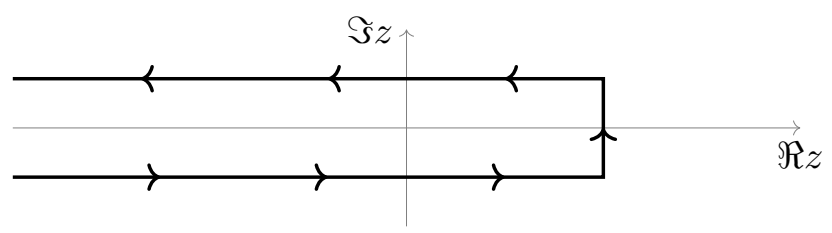

Figure 3. Contour $\mathcal{C}^{-}$.

Contour $\mathcal{C}^{-}$is a positively oriented contour consisting of the segment $[M-r \sqrt{-1}, M+r \sqrt{-1}]$ and the horizontal lines $[M-r \sqrt{-1},-\infty-r \sqrt{-1}),[M+r \sqrt{-1},-\infty+r \sqrt{-1})$, for some $-\frac{\pi}{2 \ln q}>r>0$ and $M>\lambda_{1}$, see Fig. 3. Observe that $\mathcal{C}^{-}$encloses all real poles of the integrand (which accumulate at $-\infty)$ and no other poles.

Remark 3.4. In the formulas above, $x^{z}=\exp (z \ln x)$. If $x \notin(-\infty, 0)$, we can use the principal branch of the logarithm to define $\ln x$, and if $x \in(-\infty, 0)$, then we can define the logarithm in the complex plane cut along $(-\sqrt{-1} \infty, 0]$ such that $\Im \ln a=0$ for all $a \in(0, \infty)$.

Remark 3.5. The formulas in Theorems 3.2 and 3.3 probably hold for more general contours $\mathcal{C}^{+}, \mathcal{C}^{-}$, but we do not need more generality for our purposes.

Remark 3.6. When $\theta=1$, Theorem 3.1 recovers [24, Theorem 3.6].

Remark 3.7. The infinite contours in Theorems 3.2 and 3.3 are needed because there are infinitely many real poles in the integrand and the contour needs to enclose all of them. When $\theta \in \mathbb{N}$, the integrands have finitely many poles, and we can therefore close the contours, obtaining eventually Theorem 3.1. More generally, if $\theta>0$ is such that $\theta N \in \mathbb{N}$, a similar remark applies. In fact, we can write the product of $q$-Gamma function ratios appearing in the integrand (3.2) as

$$
\begin{aligned}
& \prod_{i=1}^{N} \frac{\Gamma_{q}\left(\lambda_{i}+\theta(N-i)-z\right)}{\Gamma_{q}\left(\lambda_{i}+\theta(N-i+1)-z\right)} \\
& \quad=\frac{\Gamma_{q}\left(\lambda_{1}+\theta(N-1)-z\right)}{\Gamma_{q}\left(\lambda_{2}+\theta(N-1)-z\right)} \cdots \frac{\Gamma_{q}\left(\lambda_{N-1}+\theta-z\right)}{\Gamma_{q}\left(\lambda_{N}+\theta-z\right)} \frac{\Gamma_{q}\left(\lambda_{N}-z\right)}{\Gamma_{q}\left(\lambda_{1}+\theta N-z\right)},
\end{aligned}
$$

and since $\Gamma_{q}(t+1)=\frac{1-q^{t}}{1-q} \Gamma_{q}(t)$, we conclude that the product above is a rational function in $q^{-z}$ with finitely many real poles. Thus formula (3.2) is true if we replaced contour $\mathcal{C}^{+}$by a closed contour $\mathcal{C}_{0}$ containing all finitely many real poles of the integrand. Similarly, we can replace $\mathcal{C}^{-}$ by a closed contour $\mathcal{C}_{0}$ in (3.3).

\subsection{An example}

Before carrying out the proofs of the theorems above in full generality, we prove some very special cases, by means of the residue theorem and the $q$-binomial formula. For simplicity, let $|x|<1$ be a complex number, and consider the empty partition $\lambda=\varnothing$, or equivalently the $N$-signature 
$\lambda=\left(0^{N}\right)=(0,0, \ldots, 0)$. As remarked in Section 2.2, we have $P_{\left(0^{N}\right)}(q, t)=1$, and therefore the left-hand sides of identities (3.1) and (3.2) are both equal to 1 , when $\lambda=\left(0^{N}\right)$. Let us prove that the right-hand sides of (3.1) and (3.2) also equal 1 , for $\lambda=\left(0^{N}\right)$.

Let us begin with the case $\theta \notin \mathbb{N}$, i.e., the right-hand side of (3.2). Since the contour $\mathcal{C}^{-}$ encloses all real poles in the integrand in its interior, then the right-hand side of (3.2) equals

$$
\frac{\ln q}{1-q} \frac{\left(x t^{N} ; q\right)_{\infty}}{(x q ; q)_{\infty}} \times \Gamma_{q}(\theta N) \times \sum_{n=0}^{\infty} x^{n} \frac{\operatorname{Res}_{z=n} \Gamma_{q}(-z)}{\Gamma_{q}(\theta N-n)} .
$$

From the definition of $q$-Gamma functions, see Appendix A, it is evident that, for any $n \in \mathbb{Z}_{\geq 0}$, we have

$$
\operatorname{Res}_{z=n} \Gamma_{q}(-z)=\frac{(-1)^{n}(1-q)^{n+1}}{\ln q} \frac{q^{\left(\begin{array}{c}
n+1 \\
2
\end{array}\right)}}{(q ; q)_{n}} .
$$

Furthermore, $\Gamma_{q}(t+1)=\frac{1-q^{t}}{1-q} \Gamma_{q}(t)$ gives $\frac{\Gamma_{q}(\theta N)}{\Gamma_{q}(\theta N-n)}=(1-q)^{-n}\left(q^{\theta N-n} ; q\right)_{n}$, so (3.5) equals

$$
\frac{\left(x t^{N} ; q\right)_{\infty}}{(x q ; q)_{\infty}} \times \sum_{n=0}^{\infty} \frac{(-1)^{n} q^{\left(\begin{array}{c}
n+1 \\
2
\end{array}\right)\left(q^{\theta N-n} ; q\right)_{n}}}{(q ; q)_{n}} x^{n}
$$

The latter indeed equals 1 because of the $q$-binomial theorem, Theorem A.3, applied to $z=$ $x q^{\theta N}=x t^{N}, a=q^{1-\theta N}$, and the equality $q^{\theta N n}\left(q^{1-\theta N} ; q\right)_{n}=(-1)^{n} q^{\left({ }^{n+1}\right)}\left(q^{\theta N-n} ; q\right)_{n} \forall n \geq 0$.

Second, let us consider the case $\theta \in \mathbb{N}$, i.e., the right-hand side of (3.1). Observe that for $\lambda=\left(0^{N}\right)$, the integrand in $(3.1)$ can be rewritten as $x^{z} \cdot \prod_{i=0}^{\theta N-1}\left(1-q^{z-i}\right)^{-1}$, whose set of poles enclosed in the interior of $\mathcal{C}_{0}$ is $\{0,1,2, \ldots, \theta N-1\}$. Since $\operatorname{Res}_{z=n}\left(1-q^{z-n}\right)^{-1}=-(\ln q)^{-1}=$ $(\ln (1 / q))^{-1}$, similar considerations as above lead us to conclude that the right side of (3.1) is equal to the finite sum

$$
\begin{aligned}
& \prod_{i=1}^{\theta N-1} \frac{1-q^{i}}{x-q^{i}} \times \sum_{n=0}^{\theta N-1} \frac{x^{n}}{\prod_{\substack{0 \leq i \leq \theta N-1 \\
i \neq n}}\left(1-q^{n-i}\right)}=\prod_{i=1}^{\theta N-1} \frac{1-q^{-i}}{1-x q^{-i}} \times \sum_{n=0}^{\theta N-1} \frac{x^{n}}{(q ; q)_{n}\left(q^{-1} ; q^{-1}\right)_{\theta N-n-1}}
\end{aligned}
$$

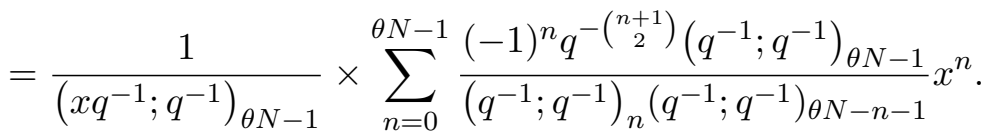

The latter equals 1, because of Corollary A.4 of the $q$-binomial formula, applied to $z=x q^{-1}$ and $M=\theta N-1$.

A simple argument involving the index stability for Macdonald polynomials, see (2.1), shows that if Theorems 3.1 and 3.2 hold for $\lambda \in \mathbb{G T}_{N}$, then they hold for $\left(\lambda_{1}+n \geq \lambda_{2}+n \geq \cdots \geq\right.$ $\left.\lambda_{N}+n\right) \in \mathbb{G}_{N}$, and any $n \in \mathbb{Z}$, cf. Step 5 in Section 3.3 below. Thus the present example shows how to prove Theorems 3.1 and 3.2 for signatures of the form $(n, n, \ldots, n) \in \mathbb{G T}_{N}$, only by use of the classical $q$-binomial theorem.

\subsection{Integral formula when $t=q^{\theta}, \theta \in \mathbb{N}$ : Proof of Theorem 3.1}

Assume $\theta \in \mathbb{N}$ and $t=q^{\theta}$. The proof of Theorem 3.1 is broken down into several steps. In the first four steps, we prove the statement for positive signatures $\lambda \in \mathbb{G T}_{N}^{+}$(when all coordinates are nonnegative: $\lambda_{1} \geq \cdots \geq \lambda_{N} \geq 0$ ), and in step 5 we extend it for all signatures $\lambda \in \mathbb{G T}_{N}$ (when some coordinates of $\lambda$ could be negative). 
Step 1. We derive a contour integral formula for the ratio $\frac{P_{\lambda}\left(q^{r} t^{N-1}, t^{N-2}, \ldots, t, 1 ; q, t\right)}{P_{\lambda}\left(t^{N-1}, \ldots, t, 1 ; q, t\right)}$ of Macdonald polynomials, and any $r \in \mathbb{N}$. The index-argument symmetry, Theorem 2.2, applied to $\lambda=$ $\left(\lambda_{1}, \ldots, \lambda_{N}\right)$ and $\mu=(r)=\left(r, 0^{N-1}\right)$, gives

$$
\frac{P_{\lambda}\left(q^{r} t^{N-1}, t^{N-2}, \ldots, t, 1 ; q, t\right)}{P_{\lambda}\left(t^{N-1}, \ldots, t, 1 ; q, t\right)}=\frac{P_{(r)}\left(q^{\lambda_{1}} t^{N-1}, \ldots, q^{\lambda_{N-1}} t, q^{\lambda_{N}} ; q, t\right)}{P_{(r)}\left(t^{N-1}, \ldots, t, 1 ; q, t\right)} .
$$

The denominator $P_{(r)}\left(t^{N-1}, \ldots, t, 1 ; q, t\right)$ has a simple expression due to the evaluation identity, Theorem 2.3; it is particularly simple for the row partition $(r)$ :

$$
P_{(r)}\left(t^{N-1}, \ldots, t, 1 ; q, t\right)=\prod_{j=2}^{N} \frac{\left(q^{r} t^{j-1} ; q\right)_{\infty}\left(t^{j} ; q\right)_{\infty}}{\left(q^{r} t^{j} ; q\right)_{\infty}\left(t^{j-1} ; q\right)_{\infty}}=\frac{\left(q^{r} t ; q\right)_{\infty}\left(t^{N} ; q\right)_{\infty}}{\left(q^{r} t^{N} ; q\right)_{\infty}(t ; q)_{\infty}}=\frac{\left(t^{N} ; q\right)_{r}}{(t ; q)_{r}}
$$

Since we also have $P_{(r)}(q, t)=\frac{(q ; q)_{r}}{(t ; q)_{r}} g_{r}(q, t)$, see (2.4), identity (3.6) becomes

$$
\frac{P_{\lambda}\left(q^{r} t^{N-1}, t^{N-2}, \ldots, t, 1 ; q, t\right)}{P_{\lambda}\left(t^{N-1}, \ldots, t, 1 ; q, t\right)}=\frac{(q ; q)_{r}}{\left(t^{N} ; q\right)_{r}} g_{r}\left(q^{\lambda_{1}} t^{N-1}, \ldots, q^{\lambda_{N}} ; q, t\right) .
$$

The symmetric polynomials $g_{r}(q, t)$, in addition to being essentially one-row Macdonald polynomials, can be defined in terms of their generating function as follows, see [32, Chapter VI]:

$$
\prod_{i=1}^{N} \frac{\left(t x_{i} y ; q\right)_{\infty}}{\left(x_{i} y ; q\right)_{\infty}}=\sum_{r \geq 0} g_{r}\left(x_{1}, \ldots, x_{N} ; q, t\right) y^{r}
$$

The relation (3.8) holds formally in the ring $\Lambda_{F}\left[x_{1}, \ldots, x_{N}\right][[y]]$. If we fix nonzero values $x_{1}, \ldots, x_{N} \in \mathbb{C} \backslash\{0\}$, the identity above is an equality of real analytic functions in the domain $\left\{y \in \mathbb{C}: \max _{1 \leq i \leq N}\left|x_{i} y\right|<1\right\}$. Thus we have the following contour integral representation

$$
g_{r}\left(x_{1}, \ldots, x_{N} ; q, t\right)=\frac{1}{2 \pi \sqrt{-1}} \oint_{C} \frac{1}{y^{r+1}} \prod_{i=1}^{N} \frac{\left(t x_{i} y ; q\right)_{\infty}}{\left(x_{i} y ; q\right)_{\infty}} \mathrm{d} y
$$

where $C$ is any circle around the origin and radius smaller than $\left(\max _{i}\left|x_{i}\right|\right)^{-1}$. Let $x_{i}=q^{\lambda_{i}} t^{N-i}$ for $i=1,2, \ldots, N$ in the integral representation of $g_{r}(q, t)$, and replace it into the right-hand side of (3.7); then

$$
\frac{P_{\lambda}\left(q^{r} t^{N-1}, t^{N-2}, \ldots, t, 1 ; q, t\right)}{P_{\lambda}\left(t^{N-1}, \ldots, t, 1 ; q, t\right)}=\frac{(q ; q)_{r}}{\left(t^{N} ; q\right)_{r}} \frac{1}{2 \pi \sqrt{-1}} \oint_{C} \frac{1}{y^{r+1}} \prod_{i=1}^{N} \frac{\left(q^{\lambda_{i}} t^{N-i+1} y ; q\right)_{\infty}}{\left(q^{\lambda_{i}} t^{N-i} y ; q\right)_{\infty}} \mathrm{d} y
$$

where $C$ can be taken to be any circle around the origin of radius smaller than 1 (we need here that $\lambda \in \mathbb{G T}_{N}^{+}$implies $q^{\lambda_{i}} t^{N-i}<1 \forall i$ ). For $t=q^{\theta}, \theta \in \mathbb{N}$, we can simplify (3.9) to

$$
\frac{P_{\lambda}\left(q^{r} t^{N-1}, t^{N-2}, \ldots, t, 1 ; q, t\right)}{P_{\lambda}\left(t^{N-1}, \ldots, t, 1 ; q, t\right)}=\frac{(q ; q)_{r}}{\left(q^{\theta N} ; q\right)_{r}} \frac{1}{2 \pi \sqrt{-1}} \oint_{C} \frac{y^{-(r+1)} \mathrm{d} y}{\prod_{i=1}^{N} \prod_{j=0}^{\theta-1}\left(1-q^{\lambda_{i}+\theta(N-i)+j} y\right)} .
$$

Step 2. We obtain a new contour integral representation by modifying (3.10). The resulting contour integral representation involves an open contour $\mathcal{C}^{+}$, that looks like that of Fig. 2 (but has a slight difference from that in Theorem 3.2).

Observe that the absolute value of the integrand in (3.10) is of order $o\left(R^{-r-1}\right)=o\left(R^{-2}\right)$, if $|y|=R$ is large. An application of Cauchy's theorem yields that the value of the integral is 


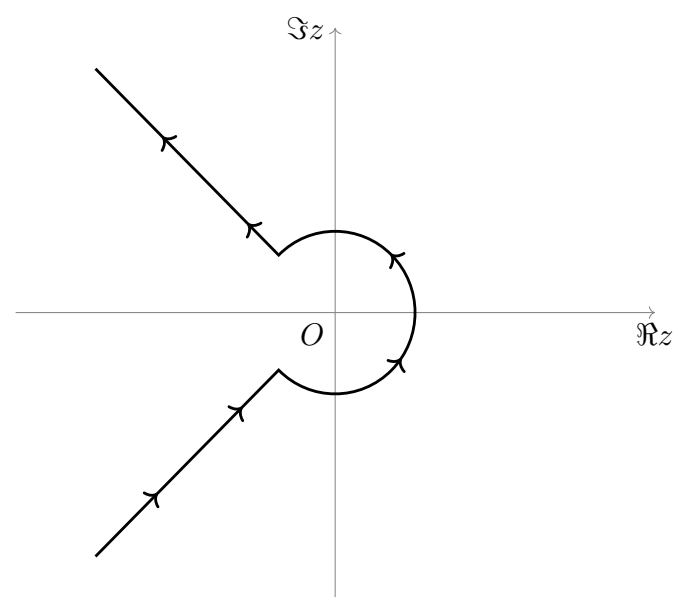

Figure 4. Contour $\mathcal{C}^{\prime}$.

unchanged if the closed contour $C$ is deformed into the "keyhole" contour $\mathcal{C}^{\prime}$ shown in Fig. 4. Let us describe the contour $\mathcal{C}^{\prime}$ in words: it is a positively oriented contour, formed by two lines away from the origin, of arguments $\pm 3 \pi / 4$, and the portion of a semicircle of some radius $0<\delta<1$. Evidently, the straight lines are part of the level lines $\Im(\ln (y))= \pm 3 \pi / 4$, while the portion of the semicircle is part of the level line $\Re(\ln (y))=\delta$ (where ln is defined in $\mathbb{C} \backslash(-\infty, 0]$ ).

Next, we make the change of variables $y=q^{-z}$, or $z=-\frac{\ln y}{\ln q}$, where $\ln$ is defined on its principal branch. Based on the previous observations about the contour $\mathcal{C}^{\prime}$ being composed by level lines, it is clear that the resulting contour for $z$ is a negatively oriented contour formed by one segment and two straight lines, but we can easily reverse the orientation of the contour at the cost of switching signs. Let us call the positively oriented contour $\mathcal{C}^{+}$, see Fig. 2; the integral formula becomes

$$
\frac{P_{\lambda}\left(q^{r} t^{N-1}, t^{N-2}, \ldots, t, 1 ; q, t\right)}{P_{\lambda}\left(t^{N-1}, \ldots, t, 1 ; q, t\right)}=\frac{(q ; q)_{r}}{\left(q^{\theta N} ; q\right)_{r}} \frac{1}{2 \pi \sqrt{-1}} \int_{\mathcal{C}^{+}} \frac{q^{(r+1) z} q^{-z} \ln q \mathrm{~d} z}{\prod_{i=1}^{N} \prod_{j=0}^{\theta-1}\left(1-q^{\lambda_{i}+\theta(N-i)+j-z}\right)}
$$

Note that points in the horizontal lines of contour $\mathcal{C}^{+}$in $(3.11)$ have imaginary parts $\pm \frac{3 \pi}{4 \ln q}$, while points in the vertical segment of $\mathcal{C}^{+}$have real part $-\ln \delta / \ln q<0$. Thus contour $\mathcal{C}^{+}$encloses exactly all the real poles of the integrand in (3.11) and no other poles (it also encloses the origin, though this not important).

Step 3. We make some final modifications to formula (3.11); the resulting contour integral representation will include a closed contour $\mathcal{C}_{0}$ as in the statement of the theorem.

Observe that the integrand in (3.11) has finitely many real poles and they are all enclosed by contour $\mathcal{C}^{+}$; also the integrand is exponentially small as $|z| \rightarrow \infty$ along the contour $\mathcal{C}^{+}$. Therefore, as an application of Cauchy's theorem, we can replace $\mathcal{C}^{+}$by a closed contour $\mathcal{C}_{0}$ that encloses all finitely many real poles of the integrand. Also note that for $r>\theta N$, we can write $\frac{(q ; q)_{r}}{\left(q^{\theta N} ; q\right)_{r}}=\prod_{i=1}^{\theta N-1} \frac{1-q^{i}}{1-q^{r+i}}$. Thus for $r>\theta N$, equation (3.11) can be rewritten as

$$
\begin{aligned}
& \frac{P_{\lambda}\left(q^{r} t^{N-1}, t^{N-2}, \ldots, t, 1 ; q, t\right)}{P_{\lambda}\left(t^{N-1}, \ldots, t, 1 ; q, t\right)} \\
& \quad=\frac{\ln q}{2 \pi \sqrt{-1}} \prod_{i=1}^{\theta N-1} \frac{1-q^{i}}{1-q^{r+i}} \oint_{\mathcal{C}_{0}} q^{r z} \prod_{i=1}^{N} \prod_{j=0}^{\theta-1} \frac{1}{1-q^{\lambda_{i}+\theta(N-i)+j-z}} \mathrm{~d} z .
\end{aligned}
$$


We let $x=q^{r} t^{N}=q^{r+\theta N}$ and replace all instances of $q^{r}$ in (3.12) above by $x / t^{N}$; then multiply both sides of the identity by $t^{|\lambda|}$. We claim that the resulting equation is exactly equality (3.1). In fact, the left-hand side of our equation is

$$
t^{|\lambda|} \frac{P_{\lambda}\left(x / t, t^{N-2}, \ldots, t, 1 ; q, t\right)}{P_{\lambda}\left(t^{N-1}, \ldots, t, 1 ; q, t\right)}=\frac{P_{\lambda}\left(x, t^{N-1}, t^{N-2}, \ldots, t ; q, t\right)}{P_{\lambda}\left(t^{N-1}, t^{N-2}, \ldots, 1 ; q, t\right)}=\frac{P_{\lambda}\left(x, t, t^{2}, \ldots, t^{N-1} ; q, t\right)}{P_{\lambda}\left(1, t, t^{2}, \ldots, t^{N-1} ; q, t\right)},
$$

by homogeneity of the Macdonald polynomials. On the other hand, the right-hand side of our equation is

$$
t^{|\lambda|} \frac{\ln q}{2 \pi \sqrt{-1}} \prod_{i=1}^{\theta N-1} \frac{1-q^{i}}{1-x q^{i-\theta N}} \oint_{\mathcal{C}_{0}} x^{z} t^{-N z} \prod_{i=1}^{N} \prod_{j=0}^{\theta-1} \frac{1}{1-q^{\lambda_{i}+\theta(N-i)+j-z}} \mathrm{~d} z,
$$

which can be shown to be equal to the right-hand side of (3.1), by simple algebraic manipulations.

The conclusion is that we have proved identity (3.1) for all $x=q^{m}$ with $m \in \mathbb{N}$ large enough (to be precise, $m$ is of the form $r+\theta N$ and $r>\theta N$, so the statement was proved for all integers $m>2 \theta N)$.

Step 4. Still assuming $\lambda \in \mathbb{G T}_{N}^{+}$, we prove Theorem 3.1 for all $x \in \mathbb{C} \backslash\left(\{0\} \cup\left\{q^{i}: i=\right.\right.$ $1,2, \ldots, \theta N-1\})$. Observe that $x \neq 0$ is imposed to make sense of the term $x^{z}=\exp (z \ln x)$ and $x \notin\left\{q, q^{2}, \ldots, q^{\theta N-1}\right\}$ is necessary so that the denominator of the right-hand side of (3.1) is nonzero.

We claim that both sides of (3.1) are rational functions of $x$. This would prove the desired result, given that we have shown in step 3 above that (3.1) holds for infinitely many points $q^{m}$, where $m$ is large enough. The left-hand side of (3.1) is obviously a polynomial on $x$. In the right-hand side of (3.1), we have the product $\prod_{i=1}^{\theta N-1}\left(x-q^{i}\right)^{-1}$ which is a rational function. We only need to check that the contour integral is also a rational function on $x$. In fact, this follows from the residue theorem and the fact that there are finitely many poles in the interior of $\mathcal{C}_{0}$, all of these being simple and integral. The fact that the poles considered above are simple and integral can be easily checked and is equivalent to fact that all the values $\lambda_{i}+\theta(N-i)+j$, for $1 \leq i \leq N, 0 \leq j \leq \theta-1$, are pairwise distinct integers.

Step 5. We extend equality (3.1) to all signatures $\lambda \in \mathbb{G}_{N}$.

Let $\lambda \in \mathbb{G T}_{N}$ be arbitrary and we aim to prove (3.1). If $\lambda \in \mathbb{G T}_{N}^{+}$, the result is already proved in the first four steps above. Otherwise, choose $m \in \mathbb{N}$ such that $\lambda_{N}+m \geq 0$, and so $\widetilde{\lambda} \stackrel{\text { def }}{=} \lambda+\left(m^{N}\right)=\left(\lambda_{1}+m, \lambda_{2}+m, \ldots, \lambda_{N}+m\right) \in \mathbb{G}_{N}^{+}$

By the index stability $(2.1),\left(x_{1} \cdots x_{N}\right)^{m} P_{\lambda}\left(x_{1}, \ldots, x_{N} ; q, t\right)=P_{\widetilde{\lambda}}\left(x_{1}, \ldots, x_{N} ; q, t\right)$. Thus multiplying the left-hand side of the equality (3.1) by $x^{m}=\left(x \cdot t \cdot t^{2} \cdots t^{N-1}\right)^{m}\left(1 \cdot t \cdot t^{2} \cdots t^{N-1}\right)^{-m}$ gives

$$
\frac{\left(x \cdot t \cdot t^{2} \cdots t^{N-1}\right)^{m} \cdot P_{\lambda}\left(x, t, t^{2}, \ldots, t^{N-1} ; q, t\right)}{\left(1 \cdot t \cdot t^{2} \cdots t^{N-1}\right)^{m} \cdot P_{\lambda}\left(1, t, t^{2}, \ldots, t^{N-1} ; q, t\right)}=\frac{P_{\widetilde{\lambda}}\left(x, t, t^{2}, \ldots, t^{N-1} ; q, t\right)}{P_{\widetilde{\lambda}}\left(1, t, t^{2}, \ldots, t^{N-1} ; q, t\right)} .
$$

If we multiply the right-hand side of equality (3.1) by $x^{m}$, and also make the change of variables $z \mapsto z-m$ in the contour integral, we obtain

$$
\ln (1 / q) \prod_{i=1}^{\theta N-1} \frac{1-q^{i}}{x-q^{i}} \frac{1}{2 \pi \sqrt{-1}} \oint_{\mathcal{C}_{0}} \frac{x^{z}}{\prod_{i=1}^{N} \prod_{j=0}^{\theta-1}\left(1-q^{z-\left(\widetilde{\lambda}_{i}+\theta(N-i)+j\right)}\right)} \mathrm{d} z
$$

Since we have proved equality (3.1) for the positive signature $\tilde{\lambda}$ already, then (3.1) also holds for $\lambda$, since we have multiplied both sides by $x^{m}$ and obtained equal expressions. 


\subsection{Integral formula for general $q, t \mathrm{I}$ : Outline of proof of Theorems 3.2 and 3.3}

Theorems 3.2 and 3.3 are analytic continuations of Theorem 3.1, with respect to the variable $\theta$. We shall need the weak version of Carlson's lemma below, which is proved in [2, Theorem 2.8.1] (in this reference, the statement is given for functions that are analytic and bounded on $\{z \in \mathbb{C}: \Re z \geq 0\}$, but a simple change of variables $z \mapsto z+M$, for some large positive integer $M \in \mathbb{N}$ leads us to the version below).

Lemma 3.8 (Carlson's lemma). Let $f(z)$ be a holomorphic function on the right half plane $\{z \in \mathbb{C}: \Re z>0\}$, such that $f(z)$ is uniformly bounded on the domain $\{z \in \mathbb{C}: \Re z \geq M\}$, for some $M>0$. If $f(n)=0$ for all $n \in \mathbb{N}$, then $f$ is identically zero.

We outline the steps of the proof of Theorem 3.2 below, and we shall carry out the detailed proof of each step in the next section. Theorem 3.3 can be proved analogously and we leave the details to the reader.

- Step 0. Prove that the right-hand side of (3.2) is well-defined, i.e., the contour integral is absolutely convergent. We prove the absolute convergence for all $|x| \leq 1, x \neq 0$, and $\theta>0$.

- Step 1. Reduce the general statement to the case $|x|<1, x \neq 0$, and $\lambda \in \mathbb{G} \mathbb{T}_{N}^{+}$. Assume the latter conditions are in place for the remaining steps in the proof.

- Step 2. Prove that the equation (3.2) of Theorem 3.2 holds when $t=q^{\theta}$ and $\theta \in \mathbb{N}$.

- Step 3. Prove that both sides of equation (3.2) are holomorphic functions of $\theta$ on the right half plane $\{\theta \in \mathbb{C}: \Re \theta>0\}$.

- Step 4. Prove that, for some (sufficiently large) $M>0$, both sides of equation (3.2) are uniformly bounded functions of $\theta$ on $\{\theta \in \mathbb{C}: \Re \theta \geq M\}$.

Then we can conclude Theorem 3.2 as follows. From steps $2-4$ and Lemma 3.8 above, identity (3.2) is proved for all $x \in \mathbb{C}$ with $|x|<1, x \neq 0, \lambda \in \mathbb{G T}_{N}^{+}, \theta \in\{z \in \mathbb{C}: \Re z>0\}$, and in particular for all $\theta>0$. Thus step 1 shows the theorem holds for the more general case $x \in \mathbb{C} \backslash\{0\},|x| \leq 1$, and $\lambda \in \mathbb{G T}_{N}$.

\subsection{Integral formula for general $q, t$ II: Proof of Theorem 3.2}

In this section, we prove Theorem 3.2; as we already mentioned, Theorem 3.3 can be proved in an analogous way, and we leave the details to the reader. From the last paragraph of the previous section, Theorem 3.2 will be proved if we verify steps $0-4$ stated there.

Proof of Step 0. Fix $\theta>0, x \in \mathbb{C} \backslash\{0\},|x| \leq 1$, and let

$$
F_{q}(z ; \theta) \stackrel{\text { def }}{=} x^{z} \prod_{i=1}^{N} \frac{\Gamma_{q}\left(\lambda_{i}+\theta(N-i)-z\right)}{\Gamma_{q}\left(\lambda_{i}+\theta(N-i+1)-z\right)} .
$$

From the definition of the $q$-Gamma function, $F_{q}(z ; \theta)$ is a holomorphic function in a neighborhood of the contour $\mathcal{C}^{+}$, thus $\int_{\mathcal{C}^{+}} F_{q}(z ; \theta) \mathrm{d} z$ is a well-posed integral. To prove its absolute convergence, it suffices to show that $\left|F_{q}(z ; \theta)\right| \leq c_{1} \cdot q^{c_{2} \Re z}$, for some $c_{1}, c_{2}>0$ and all $z \in \mathcal{C}^{+}$with $\Re z$ large enough.

Because $|x| \leq 1$ and all $z$ in the contour $\mathcal{C}^{+}$have bounded imaginary parts, we have $\left|x^{z}\right|=$ $\exp (\Re z \ln |x|-\Im z \arg (x)), z \in \mathcal{C}^{+}$, is upper-bounded by a constant. Thus it will suffice to show

$$
\left|\frac{\Gamma_{q}\left(\lambda_{i}+\theta(N-i)-z\right)}{\Gamma_{q}\left(\lambda_{i}+\theta(N-i+1)-z\right)}\right| \leq c_{1} \cdot q^{c_{2} \Re z}, \quad i=1,2, \ldots, N
$$


for some $c_{1}, c_{2}>0$ and all $z \in \mathcal{C}^{+}$with $\Re z$ large enough. Since each $\lambda_{i}+\theta(N-i)$ is real and $|\Im z|$ is constant for $z \in \mathcal{C}^{+}$, when $\Re z$ is large enough, the statement reduces to showing

$$
\left|\frac{\Gamma_{q}(-z)}{\Gamma_{q}(\theta-z)}\right| \leq c_{1} \cdot q^{c_{2} \Re z},
$$

for some $c_{1}, c_{2}>0$ and all $z \in \mathcal{C}^{+}$with $\Re z$ large enough.

Let $\pi / 2>d>0$ be the value such that $|\Im z|$, for any point $z \in \mathcal{C}^{+}$with $\Re z$ large enough, equals $-d / \ln q$ ( $\operatorname{such} d$ exists by our assumptions on the contour $\mathcal{C}^{+}$). There exists a real number $a>0$ large enough so that the following inequality holds

$$
q^{a}\left(1+q^{\theta}\right) \leq 2 \cos d
$$

Now we consider only points $z \in \mathcal{C}^{+}$with $\Re z$ large enough so that $|\Im z|=-d / \ln q$ and $\Re z>$ $\theta+a+1$; let $M=M(z)=\lfloor\Re z-\theta-a\rfloor \in \mathbb{N}$. From the definition of $a$ in (3.14), the restriction on the values of $\Re z$, and $q \in(0,1)$, one can easily obtain

$$
\left|1-q^{z-\theta-j}\right| \leq\left|1-q^{z-j}\right|, \quad \forall j=0,1, \ldots, M
$$

As a consequence of inequality (3.15) and the definition of the $q$-Gamma function, we have

$$
\begin{aligned}
& \left|\frac{\Gamma_{q}(-z)}{\Gamma_{q}(\theta-z)}\right|=\left|(1-q)^{\theta} \frac{\left(q^{-z+\theta} ; q\right)_{\infty}}{\left(q^{-z} ; q\right)_{\infty}}\right| \\
& \quad=\left|(1-q)^{\theta} \frac{\left(1-q^{\theta-z}\right) \cdots\left(1-q^{\theta-z+M}\right)}{\left(1-q^{-z}\right) \cdots\left(1-q^{-z+M}\right)} \frac{\left(q^{-z+\theta+M+1} ; q\right)_{\infty}}{\left(q^{-z+M+1} ; q\right)_{\infty}}\right| \\
& \quad=\left|(1-q)^{\theta} \frac{\left(1-q^{z-\theta}\right) \cdots\left(1-q^{z-\theta-M}\right)}{\left(1-q^{z}\right) \cdots\left(1-q^{z-M}\right)} q^{\theta(M+1)} \frac{\left(q^{\theta-z+M+1} ; q\right)_{\infty}}{\left(q^{-z+M+1} ; q\right)_{\infty}}\right| \\
& \quad \leq(1-q)^{\theta} \cdot q^{\theta(M+1)}\left|\frac{\left(q^{\theta-z+M+1} ; q\right)_{\infty}}{\left(q^{-z+M+1} ; q\right)_{\infty}}\right| .
\end{aligned}
$$

Since $M=\lfloor\Re z-\theta-a\rfloor$, the previous inequality almost shows (3.13). We are left to show that the absolute value of $\left(q^{\theta-z+M+1} ; q\right)_{\infty} /\left(q^{-z+M+1} ; q\right)_{\infty}$ is upper bounded by a constant independent of $z \in \mathcal{C}^{+}$as long as $\Re z$ is large enough. In fact, we have $\Re z-\theta-a \geq$ $M>\Re z-\theta-a-1$, so $\left|q^{\theta-z+M+1}\right|,\left|q^{-z+M+1}\right| \leq q^{-\theta-a}$ and $\left|\Im q^{\theta-z+M+1}\right|,\left|\Im q^{-z+M+1}\right| \geq$ $q^{\theta+M+1-\Re z} \sin d \geq q^{1-a} \sin d>0$. Because the $q$-Pochhammer symbol $(x ; q)_{\infty}$ is holomorphic on $x \in \mathbb{C}$, in particular continuous, the absolute value $\left|(x ; q)_{\infty}\right|$ attains a maximum $c_{\max } \geq 0$ and a minimum value $c_{\min } \geq 0$ on the compact subset $\left\{x \in \mathbb{C}:|x| \leq q^{-\theta-a},|\Im x| \geq q^{1-a} \sin d\right\}$, and moreover $c_{\min }>0$ because all the roots of $(x ; q)_{\infty}=0$ are real. We have thus proved $\left|\left(q^{\theta-z+M+1} ; q\right)_{\infty}\right| /\left|\left(q^{-z+M+1} ; q\right)_{\infty}\right| \leq c_{\max } / c_{\min }<\infty$, as desired.

Proof of Step 1. Assume we proved identity (3.2) when $x \in \mathbb{C} \backslash\{0\},|x|<1$. Then an easy application of the dominated convergence theorem (we know the integral converges absolutely when $x=1$ because of step 1 ) shows the equation would also hold for all $x \in \mathbb{C} \backslash\{0\},|x| \leq 1$.

Also, observe that step 5 of the proof of Theorem 3.1 can be repeated almost word-by-word to extend the theorem to all $\lambda \in \mathbb{G T}_{N}$, assuming that it was proved for all $\lambda \in \mathbb{G}_{N}^{+}$. Therefore we will assume for convenience in the next steps that $|x|<1, x \neq 0, \lambda \in \mathbb{G} \mathbb{T}_{N}^{+}$, and prove the theorem only in that case, without loss of generality.

Proof of Step 2. In this step, we consider the case $\theta \in \mathbb{N}$. Since we are also assuming $|x|<1, x \neq 0$, then clearly $x t^{N} \notin\left\{0, q, q^{2}, \ldots, q^{\theta N-1}\right\}$ and therefore equality (3.1) holds if $x$ was 
replaced with $x t^{N}$. After multiplying both sides of the resulting equation by $t^{-|\lambda|}$, we claim that we arrive at the desired (3.2) with $\mathcal{C}_{0}$ in place of $\mathcal{C}^{+}$. In fact, the left-hand side of our equation is

$$
\begin{aligned}
t^{-|\lambda|} & \frac{P_{\lambda}\left(x t^{N}, t, t^{2}, \ldots, t^{N-1} ; q, t\right)}{P_{\lambda}\left(1, t, t^{2}, \ldots, t^{N-1} ; q, t\right)} \\
& =\frac{P_{\lambda}\left(x t^{N-1}, 1, t, \ldots, t^{N-2} ; q, t\right)}{P_{\lambda}\left(1, t, t^{2}, \ldots, t^{N-1} ; q, t\right)}=\frac{P_{\lambda}\left(x t^{N-1}, t^{N-2}, \ldots, t, 1 ; q, t\right)}{P_{\lambda}\left(t^{N-1}, t^{N-2}, \ldots, t, 1 ; q, t\right)}
\end{aligned}
$$

thanks to the homogeneity and symmetry of Macdonald polynomials. On the other hand, the right-hand side of our equation is

$$
t^{-|\lambda|} \ln (1 / q) \prod_{i=1}^{\theta N-1} \frac{1-q^{i}}{x q^{\theta N}-q^{i}} \frac{1}{2 \pi \sqrt{-1}} \oint_{\mathcal{C}_{0}} \frac{x^{z} t^{N z}}{\prod_{i=1}^{N} \prod_{j=0}^{\theta-1}\left(1-q^{z-\left(\lambda_{i}+\theta(N-i)+j\right)}\right)} \mathrm{d} z,
$$

which can be shown equal to the right-hand side of (3.2) (with $\mathcal{C}^{+}$replaced by $\mathcal{C}_{0}$ ) after simple algebraic manipulations. Finally observe that contour $\mathcal{C}_{0}$ can be replaced by $\mathcal{C}^{+}$by an application of Cauchy's theorem.

Proof of Step 3. Let us begin by proving holomorphicity of the left-hand side of (3.2) with respect to the variable $\theta$; observe that $\theta$ only appears inside the variable $t=q^{\theta}$. The Macdonald polynomials $P_{\lambda}\left(x_{1}, \ldots, x_{N} ; q, t\right)$ are holomorphic functions of $\theta$ on $\{\theta \in \mathbb{C}: \Re \theta>$ $0\}$ because all the branching coefficients $\psi_{\mu / \nu}(q, t)$ are holomorphic on this domain. Then $P_{\lambda}\left(x t^{N-1}, t^{N-2}, \ldots, t, 1 ; q, t\right)$ and $P_{\lambda}\left(t^{N-1}, t^{N-2}, \ldots, t, 1 ; q, t\right)$ are also holomorphic. It follows that the ratio of these two quantities is holomorphic if we proved that the denominator $P_{\lambda}\left(t^{N-1}, t^{N-2}, \ldots, t, 1 ; q, t\right)$ never vanishes for $\Re \theta>0$, or equivalently for $|t|<1$; this is evident from the evaluation identity for Macdonald polynomials, Theorem 2.3.

Next we prove holomorphicity of the right-hand side of (3.2) as a function of $\theta$ in the domain $\{\theta \in \mathbb{C}: \Re \theta>0\}$. Clearly $\left(x q^{\theta N} ; q\right)_{\infty} \Gamma_{q}(\theta N)$ is holomorphic in the given domain of $\theta$, but it is less clear that the integral $\int_{\mathcal{C}^{+}} F_{q}(z, \theta) \mathrm{d} z$ is holomorphic in the right half-plane, where

$$
F_{q}(z ; \theta)=x^{z} \prod_{i=1}^{N} \frac{\Gamma_{q}\left(\lambda_{i}+\theta(N-i)-z\right)}{\Gamma_{q}\left(\lambda_{i}+\theta(N-i+1)-z\right)} .
$$

First we claim that $F_{q}(z ; \theta)$ is holomorphic on $U \times\{\theta \in \mathbb{C}: \Re \theta>0\}$, for some neighborhood $U$ of $\mathcal{C}^{+}$. Indeed, the factor $x^{z}$ is clearly entire on $z$, and does not depend on $\theta$. We can write the product of ratios of $q$-Gamma functions in the definition of $F_{q}(z ; \theta)$, as we did in Remark 3.7, see (3.4). For $1 \leq i \leq N-1$, we have

$$
\frac{\Gamma_{q}\left(\lambda_{i}+\theta(N-i)-z\right)}{\Gamma_{q}\left(\lambda_{i+1}+\theta(N-i)-z\right)}=\prod_{n=\lambda_{i+1}}^{\lambda_{i}-1}[n+\theta(N-i)-z]_{q}=\prod_{n=\lambda_{i+1}}^{\lambda_{i}-1} \frac{1-q^{n+\theta(N-i)-z}}{1-q}
$$

which is clearly holomorphic on $(z, \theta) \in \mathbb{C}^{2}$. Finally the remaining factor can be written as

$$
\frac{\Gamma_{q}\left(\lambda_{N}-z\right)}{\Gamma_{q}\left(\lambda_{1}+\theta N-z\right)}=(1-q)^{\lambda_{1}+\theta N-\lambda_{N}} \frac{\left(q^{\lambda_{1}+\theta N-z} ; q\right)_{\infty}}{\left(q^{\lambda_{N}-z} ; q\right)_{\infty}} .
$$

It is clear that $(1-q)^{\lambda_{1}+\theta N-\lambda_{N}}\left(q^{\lambda_{1}+\theta N-z} ; q\right)_{\infty}$ is holomorphic on $(z, \theta) \in \mathbb{C}^{2}$. And also there is a neighborhood $U$ of $\mathcal{C}^{+}$on which the function $\left(q^{\lambda_{N}-z} ; q\right)_{\infty}^{-1}$ of $z$ is holomorphic on $U$.

Secondly, we claim that $\int_{\mathcal{C}^{+}} F_{q}(z ; \theta) \mathrm{d} z$ is absolutely convergent and moreover $\int_{\mathcal{C}^{+}}\left|F_{q}(z ; \theta)\right| \mathrm{d} z$ is uniformly bounded on compact subsets of $\{\theta \in \mathbb{C}: \Re \theta>0\}$; this will be a consequence of the stronger statement 
Claim 3.9. Consider any compact subset $K \subset\{\theta \in \mathbb{C}: \Re \theta>0\}$. There exists a constant $M_{1}>0$, depending on $K$, such that

$$
\left|F_{q}(z ; \theta)\right|<M_{1}\left|x^{z}\right|, \quad \forall z \in \mathcal{C}^{+}, \quad \theta \in K .
$$

Let us first conclude the proof of step 3 from the claim above.

Since $|x|<1$ we have that $\left|x^{z}\right|$ decreases exponentially as $|z| \rightarrow \infty, z \in \mathcal{C}^{+}$. Then inequality (3.16) shows that $\int_{\mathcal{C}^{+}} F_{q}(z ; \theta) \mathrm{d} z$ is absolutely convergent and for all $\theta$ belonging to the compact subset $K \subset\{\theta \in \mathbb{C}: \Re \theta>0\}$, we have the bound $\int_{\mathcal{C}^{+}}\left|F_{q}(z ; \theta)\right| \mathrm{d} z<C(K)$, for some constant $C(K)>0$ that depends on $K$.

Let $T$ be any triangular contour belonging to $\{\theta \in \mathbb{C}: \Re \theta>0\}$. Then

$$
\int_{T} \int_{\mathcal{C}^{+}}\left|F_{q}(z ; \theta)\right| \mathrm{d} z \mathrm{~d} \theta \leq C(T) \int_{T}|\mathrm{~d} \theta|<\infty .
$$

By Fubini's and Cauchy's theorems, we have

$$
\int_{T} \int_{\mathcal{C}^{+}} F_{q}(z ; \theta) \mathrm{d} z \mathrm{~d} \theta=\int_{\mathcal{C}^{+}} \int_{T} F_{q}(z ; \theta) \mathrm{d} \theta \mathrm{d} z=0 .
$$

Morera's theorem implies that $\int_{\mathcal{C}^{+}} F_{q}(z ; \theta) \mathrm{d} z$ is holomorphic on $\{\theta \in \mathbb{C}: \Re \theta>0\}$, concluding step 3.

Proof of Claim 3.9. Since we have shown before that $F_{q}(z ; \theta)$ is holomorphic on $U \times\{\theta \in$ $\mathbb{C}: \Re \theta>0\}$, then it suffices to prove inequality (3.16) for all $z \in \mathcal{C}^{+}, \Re z>M_{2}$ and all $\theta \in K$, where $M_{2}$ is an arbitrarily large positive constant.

We can express the product of $q$-Gamma function ratios in the definition of $F_{q}(z ; \theta)$ as we did in (3.4). The last ratio is

$$
\frac{\Gamma_{q}\left(\lambda_{N}-z\right)}{\Gamma_{q}\left(\lambda_{1}+\theta N-z\right)}=\frac{\left[-z+\lambda_{N}-1\right]_{q} \cdots[-z+1]_{q}[-z]_{q}}{\left[-z+\lambda_{1}+\theta N-1\right]_{q} \cdots[-z+\theta N+1]_{q}[-z+\theta N]_{q}} \frac{\Gamma_{q}(-z)}{\Gamma_{q}(\theta N-z)}
$$

because we are assuming $\lambda \in \mathbb{G T}_{N}^{+}$(and so $\lambda_{1} \geq \lambda_{N} \geq 0$ ). Plugging the equality above into (3.4), we obtain

$$
\begin{aligned}
\prod_{i=1}^{N} \frac{\Gamma_{q}\left(\lambda_{i}+\theta(N-i)-z\right)}{\Gamma_{q}\left(\lambda_{i}+\theta(N-i+1)-z\right)} \\
=\prod_{i, j}[\theta(N-i)+j-z]_{q} \frac{\left[\lambda_{N}-z-1\right]_{q} \cdots[-z]_{q}}{\left[\lambda_{1}+\theta N-z-1\right]_{q} \cdots[\theta N-z]_{q}} \\
\quad \times \frac{\Gamma_{q}(-z)}{\Gamma_{q}(\theta N-z)},
\end{aligned}
$$

where the product in (3.17) is over $1 \leq i \leq N-1$ and $\lambda_{i+1} \leq j<\lambda_{i}$. The term (3.17) is of the form $P\left(q^{-z}\right) / Q\left(q^{-z}\right)$, where $P, Q \in \mathbb{C}\left(q, q^{\theta}\right)[x]$ are both polynomials of degree $\lambda_{1}$. It follows that there exist constants $M_{1}^{\prime}, M_{2}^{\prime}>0$ such that $\Re z>M_{2}^{\prime}$ implies $\left|P\left(q^{-z}\right)\right| /\left|Q\left(q^{-z}\right)\right|<M_{1}^{\prime}$.

Thus we are left to deal with (3.18), which by definition of the $q$-Gamma function equals $(1-q)^{\theta N} \frac{\left(q^{\theta N-z} ; q\right)_{\infty}}{\left(q^{-z} ; q\right)_{\infty}}$. For any $\theta$ with $\Re \theta>0$, we have $\left|(1-q)^{\theta}\right|<1$. Thus we only need to prove the existence of $M_{1}^{\prime \prime}, M_{2}^{\prime \prime}>0$ such that $z \in \mathcal{C}^{+}, \Re z>M_{2}^{\prime \prime}$, implies

$$
\left|\frac{\left(q^{\theta N-z} ; q\right)_{\infty}}{\left(q^{-z} ; q\right)_{\infty}}\right|<M_{1}^{\prime \prime}, \quad \text { for all } \quad \theta \in K
$$


There exists $a>0$ large enough such that

$$
q^{-a}\left(1-q^{N \inf _{\theta \in K} \Re \theta}\right)>2 .
$$

Note that such $a$ exists because $K \subset\{\theta \in \mathbb{C}: \Re \theta>0\}$ is compact and so $\inf _{\theta \in K} \Re \theta>0$. Now consider only $z \in \mathcal{C}^{+}$with $\Re z>a+1$, and let $M=M(z) \stackrel{\text { def }}{=}\lfloor\Re z-a\rfloor \in \mathbb{N}$. From the triangle inequality, $\left|1-q^{\theta N-z+j}\right| \leq 1+q^{N \Re \theta-\Re z+j}$ and $\left|1-q^{-z+j}\right| \geq q^{-\Re z+j}-1$. Along with condition (3.20), we deduce that $\left|1-q^{-z+j}\right| \geq\left|1-q^{\theta N-z+j}\right|$, for all $0 \leq j \leq M$. Since we can write

$$
\frac{\left(q^{\theta N-z} ; q\right)_{\infty}}{\left(q^{-z} ; q\right)_{\infty}}=\frac{\left(1-q^{\theta N-z}\right) \cdots\left(1-q^{\theta N-z+M}\right)}{\left(1-q^{-z}\right) \cdots\left(1-q^{-z+M}\right)} \cdot \frac{\left(q^{\theta N-z+M+1} ; q\right)_{\infty}}{\left(q^{-z+M+1} ; q\right)_{\infty}}
$$

it follows that

$$
\left|\frac{\left(q^{\theta N-z} ; q\right)_{\infty}}{\left(q^{-z} ; q\right)_{\infty}}\right| \leq\left|\frac{\left(q^{\theta N-z+M+1} ; q\right)_{\infty}}{\left(q^{-z+M+1} ; q\right)_{\infty}}\right|
$$

Thus we only need to show that the right-hand side of (3.21) is bounded by a constant, for all $z \in \mathcal{C}^{+}$with $\Re z$ large enough.

Since $M=\lfloor\Re z-a\rfloor>\Re z-a-1$, we have $\left|q^{-z+M+1}\right|=q^{M+1-\Re z} \leq q^{-a}$ and $\left|q^{\theta N-z+M+1}\right| \leq$ $q^{N \Re \theta-a}<q^{-a}$. Moreover, for $z \in \mathcal{C}^{+}$with $\Re z$ large enough, $\left|\Im q^{-z+M+1}\right| \geq q^{-a+1}|\sin (\ln q \cdot \Im z)| \stackrel{\text { def }}{=}$ $m_{1}(z)$ and since $|\Im z|$ is a constant between 0 and $-\frac{\pi}{2 \ln q}$ for $z \in \mathcal{C}^{+}$with $\Re z$ large enough, then $m_{1}(z)=m_{1}>0$ is a strictly positive constant independent of $z \in \mathcal{C}^{+}$as long as $\Re z$ is large enough. Since the function $(x ; q)_{\infty}$ is continuous on $x \in \mathbb{C}$, we have $c_{\max } \stackrel{\text { def }}{=} \sup _{|x| \leq q^{-a}}\left|(x ; q)_{\infty}\right|<\infty$, and $c_{\text {min }} \stackrel{\text { def }}{=} \inf _{|x| \leq q^{-a},|\Im x| \geq m_{1}}\left|(x ; q)_{\infty}\right| \in(0, \infty)$. Thus the right-hand side of (3.21) is upper bounded by the constant $c_{\max } / c_{\min }<\infty$.

Proof of Step 4. We prove a stronger statement than step 4. Let $M>0$ be any positive number. We show that both sides of (3.2) are uniformly bounded on $\{\theta \in \mathbb{C}: \Re \theta \geq M\}$. Let us begin with the left-hand side of (3.2). Observe that $\theta$ appears in the left side only within the variable $t=q^{\theta}$ and $|t|=q^{\Re \theta} \leq q^{M}$. Name $\epsilon=q^{M} \in(0,1)$; we have to prove that there exists a constant $C>0$ such that

$$
\sup _{|t| \leq \epsilon}\left|\frac{P_{\lambda}\left(x t^{N-1}, t^{N-2}, \ldots, t, 1 ; q, t\right)}{P_{\lambda}\left(t^{N-1}, t^{N-2}, \ldots, t, 1 ; q, t\right)}\right| \leq C .
$$

Thanks to the branching rule for Macdonald polynomials, Theorem 2.5, and the assumptions $|x| \leq 1, x \neq 0, \lambda \in \mathbb{G} \mathbb{T}_{N}^{+}$, we have

$$
\left|\frac{P_{\lambda}\left(x t^{N-1}, t^{N-2}, \ldots, t, 1 ; q, t\right)}{P_{\lambda}\left(t^{N-1}, t^{N-2}, \ldots, t, 1 ; q, t\right)}\right| \leq \sum_{\mu \prec \lambda}\left|\psi_{\lambda / \mu}(q, t)\right||t|^{(N-1)(|\lambda|-|\mu|)}\left|\frac{P_{\mu}\left(t^{N-2}, \ldots, t, 1 ; q, t\right)}{P_{\lambda}\left(t^{N-1}, \ldots, t, 1 ; q, t\right)}\right| .
$$

Given $\lambda \in \mathbb{G} \mathbb{T}_{N}^{+}$, there are finitely many $\mu \in \mathbb{G T}_{N-1}^{+}$with $\mu \prec \lambda$. Thus it suffices to prove that there exist constants $C_{1}, C_{2}>0$ such that

$$
\left|\psi_{\lambda / \mu}(q, t)\right| \leq C_{1}, \quad\left|t^{(N-1)(|\lambda|-|\mu|)} \frac{P_{\mu}\left(t^{N-2}, \ldots, t, 1 ; q, t\right)}{P_{\mu}\left(t^{N-1}, \ldots, t, 1 ; q, t\right)}\right| \leq C_{2},
$$

where $C_{1}, C_{2}$ do not depend on $t$, though they may depend on $\mu$. 
The branching coefficient $\left|\psi_{\lambda / \mu}(q, t)\right|$, due to the expression in Theorem 2.5, is a finite product of terms of the form $\frac{1-q^{a} t^{b}}{1-q^{c} t^{d}}$, with $a, b, c, d \in \mathbb{Z}_{\geq 0}$ and $(c, d) \neq(0,0)$. We have

$$
\left|\frac{1-q^{a} t^{b}}{1-q^{c} t^{d}}\right| \leq 2\left(1-q^{c} \epsilon^{d}\right)^{-1}
$$

for all $|t| \leq \epsilon$ and $2\left(1-q^{c} \epsilon^{d}\right)^{-1}$ does not depend on $t$, so the boundedness of $\left|\psi_{\lambda / \mu}(q, t)\right|$ follows.

Due to the evaluation identity for Macdonald polynomials, Theorem 2.3, we have

$$
\begin{aligned}
& t^{(N-1)(|\lambda|-|\mu|)} \frac{P_{\mu}\left(t^{N-2}, \ldots, t, 1 ; q, t\right)}{P_{\lambda}\left(t^{N-1}, \ldots, t, 1 ; q, t\right)}=t^{(N-1)(|\lambda|-|\mu|)+n(\mu)-n(\lambda)} \\
& \quad \times \prod_{1 \leq i<j \leq N-1} \frac{\left(q^{\mu_{i}-\mu_{j}} t^{j-i} ; q\right)_{\infty}\left(t^{j-i+1} ; q\right)_{\infty}}{\left(t^{j-i} ; q\right)_{\infty}\left(q^{\mu_{i}-\mu_{j}} t^{j-i+1} ; q\right)_{\infty}} \prod_{1 \leq i<j \leq N} \frac{\left(t^{j-i} ; q\right)_{\infty}\left(q^{\lambda_{i}-\lambda_{j}} t^{j-i+1} ; q\right)_{\infty}}{\left(q^{\lambda_{i}-\lambda_{j}} t^{j-i} ; q\right)_{\infty}\left(t^{j-i+1} ; q\right)_{\infty}},
\end{aligned}
$$

where $n(\lambda) \stackrel{\text { def }}{=}(N-1) \lambda_{N}+(N-2) \lambda_{N-1}+\cdots+2 \lambda_{3}+\lambda_{2}$ and similarly for $n(\mu)$.

The last two terms above are products of a finite number of fractions $\frac{1-q^{a} t^{b}}{1-q^{c} t^{d}}$, with $a, b, c, d \in$ $\mathbb{Z}_{\geq 0},(c, d) \neq(0,0)$, and as we saw above it is implied that the absolute value of the last two terms above are upper bounded by a constant independent of $t$ (as long as $|t| \leq \epsilon$ ). Thus our only goal is to show there is an upper bound for $t^{(N-1)(|\lambda|-|\mu|)+n(\mu)-n(\lambda)}$; this fact follows if the exponent is nonnegative. In fact, we have

$$
\begin{aligned}
& (N-1)(|\lambda|-|\mu|)-(n(\lambda)-n(\mu)) \\
& \quad=(N-1)(|\lambda|-|\mu|)-\left((N-1) \lambda_{N}+(N-2)\left(\lambda_{N-1}-\mu_{N-1}\right)+\cdots+\left(\lambda_{2}-\mu_{2}\right)\right) \\
& \quad \geq(N-1)(|\lambda|-|\mu|)-(N-1)\left(\lambda_{N}+\lambda_{N-1}-\mu_{N-1}+\cdots+\lambda_{2}-\mu_{2}\right) \\
& \quad=(N-1)\left(\lambda_{1}-\mu_{1}\right) \geq 0 .
\end{aligned}
$$

Let us proceed to prove uniform boundedness of the right-hand side of $(3.2)$ on $\{\theta \in \mathbb{C}: \Re \theta \geq$ $M\}$. First of all, the triangular inequality gives

$$
\begin{aligned}
& \left|\left(x t^{N} ; q\right)_{\infty}\right| \leq \prod_{i=0}^{\infty}\left(1+|x| q^{N \Re \theta+i}\right) \leq \prod_{i=0}^{\infty}\left(1+|x| q^{i}\right), \\
& \left|(x q ; q)_{\infty}\right|=\prod_{i=1}^{\infty}\left|\left(1-x q^{i}\right)\right| \geq \prod_{i=1}^{\infty}\left(1-|x| q^{i}\right),
\end{aligned}
$$

so the factor $\left(x t^{N} ; q\right)_{\infty} /(x q ; q)_{\infty}$ in $(3.2)$ has an upper-bounded absolute value. We are left to deal with

$$
\begin{aligned}
\Gamma_{q}(\theta N) \int_{\mathcal{C}^{+}} x^{z} \prod_{i=1}^{N} \frac{\Gamma_{q}\left(\lambda_{i}+\theta(N-i)-z\right)}{\Gamma_{q}\left(\lambda_{i}+\theta(N-i+1)-z\right)} \mathrm{d} z \\
\quad=\int_{\mathcal{C}^{+}} x^{z} \Gamma_{q}(\theta N) \prod_{i=1}^{N} \frac{\Gamma_{q}\left(\lambda_{i}+\theta(N-i)-z\right)}{\Gamma_{q}\left(\lambda_{i}+\theta(N-i+1)-z\right)} \mathrm{d} z
\end{aligned}
$$

and prove its absolute value is uniformly bounded on $\{\theta \in \mathbb{C}: \Re \theta \geq M\}$.

For any $M_{2}>0$, the contribution of the portion $\mathcal{C}^{+} \cap\left\{z \in \mathbb{C}: \Re z \leq M_{2}\right\}$ of the contour is bounded by a constant. In fact, $\Re \theta \geq M$ implies $|t|=\left|q^{\theta}\right|=q^{\Re \theta} \leq q^{M}$ and $\theta$ appears in the integrand of (3.2) only as part of the exponent of some $q$, thus the integrand can be written as a function of $z$ and $t$ (with $q \in(0,1)$ fixed). Thus for $(z, t)$ in the compact subset $\left(\mathcal{C}^{+} \cap\left\{z \in \mathbb{C}: \Re z \leq M_{2}\right\}\right) \times\left[0, q^{M}\right]$, the integrand in (3.22) attains a maximum value $L<\infty$, 
and the contribution of the integral in the portion $\mathcal{C}^{+} \cap\left\{z \in \mathbb{C}: \Re z \leq M_{2}\right\}$ of the contour is upper bounded by $L$ times the length of that finite portion.

Since $|x|<1$, the term $x^{z}$ decreases exponentially as $|z| \rightarrow \infty, z \in \mathcal{C}^{+}$. Thus to deal with the infinite portion of the integral $\mathcal{C}^{+} \cap\left\{z \in \mathbb{C}: \Re z \geq M_{2}\right\}$, it is enough to show that

$$
\Gamma_{q}(\theta N) \prod_{i=1}^{N} \frac{\Gamma_{q}\left(\lambda_{i}+\theta(N-i)-z\right)}{\Gamma_{q}\left(\lambda_{i}+\theta(N-i+1)-z\right)}=(1-q) \frac{(q ; q)_{\infty}}{\left(q^{\theta N} ; q\right)_{\infty}} \prod_{i=1}^{N} \frac{\left(q^{\lambda_{i}+\theta(N-i+1)-z} ; q\right)_{\infty}}{\left(q^{\lambda_{i}+\theta(N-i)-z} ; q\right)_{\infty}}
$$

has bounded absolute value for all $z \in \mathcal{C}^{+}, \Re z>M_{2}$ and $\theta \in \mathbb{C}, \Re \theta \geq M$, for a suitable constant $M_{2}>0$. Clearly $\left|\left(q^{\theta N} ; q\right)\right|^{-1} \leq\left(q^{N \Re \theta} ; q\right)^{-1} \leq\left(q^{N M} ; q\right)^{-1}$, thus we only need a bound on the absolute value of

$$
\prod_{i=1}^{N} \frac{\left(q^{\lambda_{i}+\theta(N-i+1)-z} ; q\right)_{\infty}}{\left(q^{\lambda_{i}+\theta(N-i)-z} ; q\right)_{\infty}}
$$

uniformly over all $z \in \mathcal{C}^{+}, \Re z>M_{2}$, and $\theta \in \mathbb{C}, \Re \theta \geq M$. We can bound the absolute value of (3.23) by

$$
\begin{aligned}
& \prod_{i=1}^{N-1}\left|\frac{\left(q^{\lambda_{i+1}+\theta(N-i)-z} ; q\right)_{\infty}}{\left(q^{\lambda_{i}+\theta(N-i)-z} ; q\right)_{\infty}}\right| \times\left|\frac{\left(q^{\lambda_{1}+\theta N-z} ; q\right)_{\infty}}{\left(q^{\lambda_{N}-z} ; q\right)_{\infty}}\right| \\
& \quad=\prod_{\substack{1 \leq i \leq N-1 \\
\lambda_{i+1} \leq j<\lambda_{i}}}\left|1-q^{\theta(N-i)+j-z}\right| \times \frac{1}{\prod_{\lambda_{N} \leq j<\lambda_{1}}\left|1-q^{j-z}\right|} \times\left|\frac{\left(q^{\lambda_{1}+\theta N-z} ; q\right)_{\infty}}{\left(q^{\lambda_{1}-z} ; q\right)_{\infty}}\right| \\
& \quad \leq \prod_{\substack{1 \leq i \leq N-1 \\
\lambda_{i+1} \leq j<\lambda_{i}}}\left(1+q^{(N-i) \Re \theta+j-\Re z}\right) \times \frac{1}{\prod_{\lambda_{N} \leq j<\lambda_{1}}\left(q^{j-\Re z}-1\right)} \times\left|\frac{\left(q^{\lambda_{1}+\theta N-z} ; q\right)_{\infty}}{\left(q^{\lambda_{1}-z} ; q\right)_{\infty}}\right| \\
& \quad \leq \prod_{\lambda_{N} \leq j<\lambda_{1}} \frac{1+q^{j-\Re z}}{q^{j-\Re z}-1} \times\left|\frac{\left(q^{\lambda_{1}+\theta N-z} ; q\right)_{\infty}}{\left(q^{\lambda_{1}-z} ; q\right)_{\infty}}\right| .
\end{aligned}
$$

If $\Re z$ is large enough, the product $\prod_{\lambda_{N} \leq j<\lambda_{1}} \frac{1+q^{j-\Re z}}{q^{j-\Re z}-1}$ is clearly upper bounded by a constant. We still have to bound $\left|\frac{\left(q^{\lambda_{1}+\theta N-z} ; q\right)_{\infty}}{\left(q^{\lambda_{1}-z} ; q\right)_{\infty}}\right|$. Since $\lambda_{1}$ is real and $\Im z$ is constant for $z \in \mathcal{C}^{+}, \Re z$ large enough, it suffices to prove the following statement: there exist constants $M_{1}, M_{2}>0$ such that $z \in \mathcal{C}^{+}, \Re z>M_{2}$, and $\Re \theta \geq M$ imply

$$
\left|\frac{\left(q^{\theta N-z} ; q\right)_{\infty}}{\left(q^{-z} ; q\right)_{\infty}}\right|<M_{1}
$$

This statement was proved above in step 3, see (3.19). In that case, $\theta$ varied over a compact subset $K \subset\{\theta \in \mathbb{C}: \Re \theta>0\}$, but in this case $\theta$ varies over a closed infinite domain of the form $\{\theta \in \mathbb{C}: \Re \theta \geq M\}$. However, the expression $\frac{\left(q^{\theta N-z} ; q\right)_{\infty}}{\left(q^{-z} ; q\right)_{\infty}}$ depends on $\theta$ only by means of $q^{\theta}$, so the proof of (3.19) above can be repeated word-by-word, since we only used $|t| \leq q^{\inf _{\theta \in K} \Re \theta}<1$ in that proof.

\section{Multiplicative formulas for Macdonald characters}

We now come to the multiplicative formulas. All of our results require parameter $\theta$ to be a positive integer. In this section, $q$ is typically a variable (but of course, we can specialize $q$ to a complex number later). 


\subsection{Statement of the multiplicative theorem and some consequences}

We need some non-standard terminology on $q$-difference operators. The q-shift operators $\left\{T_{q, x_{i}}: i=1, \ldots, m\right\}$ are linear operators on $\mathbb{C}(q)\left[x_{1}, \ldots, x_{m}\right]$ that act as

$$
\left(T_{q, x_{i}} f\right)\left(x_{1}, \ldots, x_{m}\right) \stackrel{\text { def }}{=} f\left(x_{1}, \ldots, x_{i-1}, q x_{i}, x_{i+1}, \ldots, x_{m}\right) .
$$

The $q$-degree operators $\left\{D_{q, x_{i}}\right\}_{i=1, \ldots, m}$ are linear operators on $\mathbb{C}(q)\left[x_{1}, \ldots, x_{m}\right]$ defined by

$$
\begin{aligned}
& D_{q, x_{i}} \stackrel{\text { def }}{=} \frac{T_{q, x_{i}}-1}{q-1} \quad \text { or } \\
& \left(D_{q, x_{i}} f\right)\left(x_{1}, \ldots, x_{m}\right)=\frac{f\left(x_{1}, \ldots, x_{i-1}, q x_{i}, x_{i+1}, \ldots, x_{m}\right)-f\left(x_{1}, \ldots, x_{m}\right)}{q-1} .
\end{aligned}
$$

The $q$-difference operators that appear in the multiplicative formulas for Macdonald polynomials are finite sums of terms

$$
c_{i_{1}, \ldots, i_{m}}\left(x_{1}, \ldots, x_{m} ; q\right) T_{q, x_{1}}^{i_{1}} \cdots T_{q, x_{m}}^{i_{m}} \quad \text { or } \quad c_{i_{1}, \ldots, i_{m}}\left(x_{1}, \ldots, x_{m} ; q\right) D_{q, x_{1}}^{i_{1}} \cdots D_{q, x_{m}}^{i_{m}},
$$

where $\left(i_{1}, \ldots, i_{m}\right)$ vary over a finite subset of $\mathbb{Z}_{\geq 0}^{m}$, and $c_{i_{1}, \ldots, i_{m}}\left(x_{1}, \ldots, x_{m} ; q\right)$ are rational functions in the variables $q, x_{1}, \ldots, x_{m}$. Thus the operators that we consider are linear operators $\mathbb{C}(q)\left[x_{1}, \ldots, x_{m}\right] \longrightarrow \mathbb{C}\left(q, x_{1}, \ldots, x_{m}\right)$ that act on polynomials and yield rational functions.

Recall the setting of Jacobi Trudi's formula for Macdonald polynomials, Theorem 2.7. The expressions $C_{\tau_{1}, \ldots, \tau_{n}}^{\left(q, q^{\theta}\right)}\left(u_{1}, \ldots, u_{n}\right)$ were defined in $(2.5)$; they are rational functions in $q, u_{1}, \ldots, u_{n}$ whose denominators are products of linear factors. We define $M_{\theta}^{(m)}$ as the set of strictly uppertriangular $m \times m$ matrices whose entries belong to $\{0,1, \ldots, \theta\}$ (the cardinality of $M_{\theta}^{(m)}$ is $\left.(\theta+1)\left(\begin{array}{c}m \\ 2\end{array}\right)\right)$. For any strictly upper-triangular $m \times m$ matrix $\tau$ and $1 \leq i \leq m$, let $\tau_{i}^{+} \stackrel{\text { def }}{=} \sum_{j=i+1}^{m} \tau_{i, j}$ (resp. $\tau_{i}^{-} \stackrel{\text { def }}{=} \sum_{j=1}^{i-1} \tau_{j, i}$ ) be the sum of the entries of $\tau$ to the right of $(i, i)$ (resp. sum of entries of $\tau$ above $(i, i))$, cf. (2.6). The main theorem of this section is the following.

Theorem 4.1. Let $\theta \in \mathbb{N}, t=q^{\theta}, N \in \mathbb{N}, \lambda \in \mathbb{G}_{N}$. Then

$$
\begin{aligned}
& P_{\lambda}\left(x_{1}, \ldots, x_{m} ; N, q, q^{\theta}\right)=\frac{q^{\theta^{2}\left(\begin{array}{c}
m+1 \\
3
\end{array}\right)-\left\{N \theta^{2}-\left(\begin{array}{c}
\theta+1 \\
2
\end{array}\right)\right\}\left(\begin{array}{c}
m \\
2
\end{array}\right)} \prod_{i=1}^{m}[\theta(N-i+1)-1]_{q} !}{\prod_{i=1}^{m} \prod_{j=1}^{\theta(N-m+1)-1}\left(x_{i}-q^{j-\theta}\right)} \\
& \quad \times \frac{1}{\prod_{\substack{1 \leq i<j \leq m \\
0 \leq k<\theta}}\left(x_{i}-q^{k} x_{j}\right)} \times \mathcal{D}_{q, \theta}^{(m)}\left\{\prod_{i=1}^{m} \frac{P_{\lambda}\left(x_{i} ; N, q, q^{\theta}\right) \prod_{j=1}^{\theta N-1}\left(x_{i}-q^{j-\theta}\right)}{[\theta N-1]_{q} !}\right\}
\end{aligned}
$$

where $\mathcal{D}_{q, \theta}^{(m)}$ is the q-difference operator $\mathbb{C}(q)\left[x_{1}, \ldots, x_{m}\right] \longrightarrow \mathbb{C}\left(q, x_{1}, \ldots, x_{m}\right)$ given by

$$
\mathcal{D}_{q, \theta}^{(m)} \stackrel{\text { def }}{=} \frac{1}{(q-1)^{\theta\left(\begin{array}{c}
m \\
2
\end{array}\right)}} \sum_{\tau \in M_{\theta}^{(m)}}\left\{C_{\tau}^{\left(q, q^{\theta}\right)}\left(x_{1}, \ldots, x_{m}\right) \prod_{i=1}^{m} T_{q, x_{i}}^{(i-1) \theta+\tau_{i}^{+}-\tau_{i}^{-}}\right\}
$$

where for any $\tau \in M_{\theta}^{(m)}$, we denoted

$$
C_{\tau}^{\left(q, q^{\theta}\right)}\left(x_{1}, \ldots, x_{m}\right)
$$




$$
\stackrel{\text { def }}{=} \prod_{s=1}^{m-1} C_{\tau_{1, s+1}, \ldots, \tau_{s, s+1}}^{\left(q, q^{\theta}\right)}\left(\left\{u_{i}=x_{s+1}^{-1} x_{i} q^{-\theta+\sum_{j=s+2}^{m}\left(\tau_{i, j}-\tau_{s+1, j}\right)}: 1 \leq i \leq s\right\}\right) .
$$

The proof of Theorem 4.1 is given in the next subsection. We derive here some conclusions, namely two special cases when the operator $\mathcal{D}_{q, \theta}^{(m)}$ has a simple form. The first simple case is $m=2$.

Corollary 4.2. In the same setting as Theorem 4.1 (for $m=2)$, we have

$$
\begin{aligned}
P_{\lambda}\left(x_{1}, x_{2} ; N, q, q^{\theta}\right)= & \frac{q^{-(N-1) \theta^{2}+\left(\begin{array}{c}
\theta+1 \\
2
\end{array}\right) \cdot[\theta(N-1)-1]_{q} !}}{\prod_{j=1}^{N-1)-1}\left(x_{1}-q^{j-\theta}\right)\left(x_{2}-q^{j-\theta}\right)} \\
& \times \widetilde{\mathcal{D}}_{q, \theta}^{(2)}\left\{\frac{\prod_{i=1}^{2} P_{\lambda}\left(x_{i} ; N, q, q^{\theta}\right) \prod_{j=1}^{\theta N-1}\left(x_{i}-q^{j-\theta}\right)}{[\theta N-1]_{q} !}\right\}
\end{aligned}
$$

where

$$
\begin{aligned}
\widetilde{\mathcal{D}}_{q, \theta}^{(2)} & \stackrel{\text { def }}{=}\left(\frac{1}{x_{1}-x_{2}} \circ\left(D_{q, x_{2}}-D_{q, x_{1}}\right)\right)^{\theta} \\
& =\underbrace{\left(\frac{1}{x_{1}-x_{2}} \circ\left(D_{q, x_{2}}-D_{q, x_{1}}\right)\right) \circ \cdots \circ\left(\frac{1}{x_{1}-x_{2}} \circ\left(D_{q, x_{2}}-D_{q, x_{1}}\right)\right)}_{\text {composition of } \theta \text { operators }} .
\end{aligned}
$$

Proof. For $m=2$, we have $M_{\theta}^{(2)}=\left\{\left[\begin{array}{ll}0 & n \\ 0 & 0\end{array}\right], n \in\{0,1, \ldots, \theta\}\right\}$, and thus the operator $\mathcal{D}_{q, \theta}^{(2)}$ becomes

$$
\mathcal{D}_{q, \theta}^{(2)}=\frac{1}{(q-1)^{\theta}} \sum_{n=0}^{\theta}\left\{C_{n}^{\left(q, q^{\theta}\right)}\left(x_{2}^{-1} x_{1} q^{-\theta}\right) T_{q, x_{1}}^{n} T_{q, x_{2}}^{\theta-n}\right\} .
$$

We let

$$
a_{n}^{(\theta)}=\frac{C_{n}^{\left(q, q^{\theta}\right)}\left(x_{2}^{-1} x_{1} q^{-\theta}\right)}{\prod_{i=0}^{\theta-1}\left(x_{1}-q^{i} x_{2}\right)}, \quad 0 \leq n \leq \theta
$$

The statement of the theorem can be easily reduced to prove that the coefficient of $T_{q, x_{1}}^{n} T_{q, x_{2}}^{\theta-n}$ in the product

$$
D_{q, \theta}=\left(\frac{1}{x_{1}-x_{2}} \circ\left(T_{q, x_{2}}-T_{q, x_{1}}\right)\right)^{\theta}=\sum_{n=0}^{\theta} b_{n}^{(\theta)} T_{q, x_{1}}^{n} T_{q, x_{2}}^{\theta-n}
$$

is equal to $a_{n}^{(\theta)}$, i.e., we prove $a_{n}^{(\theta)}=b_{n}^{(\theta)}$ for all $\theta, n \in \mathbb{N}, 0 \leq n \leq \theta$, and we do it by induction on $\theta$. The case $\theta=1$ can be easily dealt with, using Lemma B.2. Now assume $a_{n}^{(\theta-1)}=b_{n}^{(\theta-1)}$ for all $0 \leq n \leq \theta-1$, and some $\theta \geq 2$. We prove $a_{n}^{(\theta)}=b_{n}^{(\theta)}$ for all $0 \leq n \leq \theta$. Evidently, $D_{q, \theta}=\left(\frac{1}{x_{1}-x_{2}} \circ\left(T_{q, x_{2}}-T_{q, x_{1}}\right)\right) \circ D_{q, \theta-1}$ implies

$$
b_{n}^{(\theta)}=\frac{1}{x_{1}-x_{2}}\left(T_{q, x_{2}} b_{n}^{(\theta-1)}-T_{q, x_{1}} b_{n-1}^{(\theta-1)}\right), \quad n=1,2, \ldots, \theta-1,
$$




$$
b_{0}^{(\theta)}=\frac{1}{x_{1}-x_{2}} T_{q, x_{2}} b_{0}^{(\theta-1)}, \quad b_{\theta}^{(\theta)}=\frac{1}{x_{2}-x_{1}} T_{q, x_{1}} b_{\theta-1}^{(\theta-1)} .
$$

From Lemma B.3 in Appendix B, the terms $a_{n}^{(\theta)}$ satisfy

$$
\begin{aligned}
& a_{n}^{(\theta)}=\frac{1}{x_{1}-x_{2}}\left(T_{q, x_{2}} a_{n}^{(\theta-1)}-T_{q, x_{1}} a_{n-1}^{(\theta-1)}\right), \quad n=1,2, \ldots, \theta-1, \\
& a_{0}^{(\theta)}=\frac{1}{\prod_{i=0}^{\theta-1}\left(x_{1}-q^{i} x_{2}\right)}, \quad a_{\theta}^{(\theta)}=\frac{1}{\prod_{i=0}^{\theta-1}\left(x_{2}-q^{i} x_{1}\right)} .
\end{aligned}
$$

It is not difficult to conclude from these relations, and the inductive hypothesis $a_{m}^{(\theta-1)}=b_{m}^{(\theta-1)}$ $\forall m=0,1, \ldots, \theta-1$, that $a_{n}^{(\theta)}=b_{n}^{(\theta)}$ for all $0 \leq n \leq \theta$, as desired.

When $\theta=1$ (equivalently $t=q$ ), the result has a compact form as well. Let us recall that when $t=q$, the Macdonald polynomials become the well known Schur polynomials $s_{\lambda}\left(x_{1}, \ldots, x_{N}\right)=$ $P_{\lambda}\left(x_{1}, \ldots, x_{N} ; q, q\right)$. The Schur (Laurent) polynomials $s_{\lambda}\left(x_{1}, \ldots, x_{N}\right), \lambda \in \mathbb{G T}_{N}$, can also be defined by the simple determinantal formula

$$
s_{\lambda}\left(x_{1}, \ldots, x_{N}\right)=\frac{\operatorname{det}\left[x_{i}^{N+1-j}\right]_{i, j=1}^{N}}{\prod_{1 \leq i<j \leq N}\left(x_{i}-x_{j}\right)}
$$

For any $m \in \mathbb{N}$ with $1 \leq m \leq N$, we consider

$$
s_{\lambda}\left(x_{1}, \ldots, x_{m} ; N, q\right) \stackrel{\text { def }}{=} \frac{s_{\lambda}\left(x_{1}, \ldots, x_{m}, 1, q, \ldots, q^{N-1-m}\right)}{s_{\lambda}\left(1, q, \ldots, q^{N-1}\right)}
$$

and call it a $q$-Schur character of rank $N$, number of variables $m$ and parametrized by $\lambda$; it was defined before in [21]. We recover the following theorem.

Corollary 4.3 ([24, Theorem 3.5]). Let $N \in \mathbb{N}, \lambda \in \mathbb{G T}_{N}$. Then

$$
\begin{aligned}
s_{\lambda}\left(x_{1}, \ldots, x_{m} ; N, q\right)= & \frac{q^{\left(\begin{array}{c}
m+1 \\
3
\end{array}\right)-(N-1)\left(\begin{array}{c}
m \\
2
\end{array}\right) \prod_{i=1}^{m}[N-i]_{q} !}}{\prod_{i=1}^{m} \prod_{j=1}^{N-m}\left(x_{i}-q^{j-1}\right)} \frac{1}{\Delta\left(x_{1}, \ldots, x_{m}\right)} \\
& \times \mathcal{D}_{q}^{(m)}\left\{\prod_{i=1}^{m} \frac{s_{\lambda}\left(x_{i} ; N, q\right) \prod_{j=1}^{N-1}\left(x_{i}-q^{j-1}\right)}{[N-1]_{q} !}\right\},
\end{aligned}
$$

where

$$
\mathcal{D}_{q}^{(m)} \stackrel{\text { def }}{=} \operatorname{det}\left[D_{q, x_{i}}^{j-1}\right]_{i, j=1}^{m}=\prod_{1 \leq i<j \leq m}\left(D_{q, x_{j}}-D_{q, x_{i}}\right) .
$$

Proof. Letting $\theta=1$ in Theorem 4.1, we see that the equation above holds if $\mathcal{D}_{q, 1}^{(m)}=\mathcal{D}_{q}^{(m)}$. In fact, thanks to Lemma B.2, we have

$$
\mathcal{D}_{q, 1}^{(m)}=(q-1)^{-\left(\begin{array}{c}
m \\
2
\end{array}\right)} \sum_{\tau \in M_{1}^{(m)}} \prod_{s=1}^{m-1}(-1)^{\tau_{1, s+1}+\cdots+\tau_{s, s+1}} \prod_{i=1}^{m} T_{q, x_{i}}^{i-1+\tau_{i}^{+}-\tau_{i}^{-}} .
$$


Evidently $\mathcal{D}_{q}^{(m)}=\prod_{i<j}\left(D_{q, x_{j}}-D_{q, x_{i}}\right)=(q-1)^{-\left(\begin{array}{c}m \\ 2\end{array}\right)} \prod_{i<j}\left(T_{q, x_{j}}-T_{q, x_{i}}\right)$, thus we need to show

$$
\prod_{1 \leq i<j \leq m}\left(T_{q, x_{j}}-T_{q, x_{i}}\right)=\sum_{\tau \in M_{1}^{(m)}}(-1)^{|\tau|} \prod_{k=1}^{m} T_{q, x_{k}}^{k-1+\tau_{k}^{+}-\tau_{k}^{-}}
$$

where we denoted by $|\tau|$ to the sum of all entries of $\tau \in M_{1}^{(m)}$. The operators $T_{q, x_{1}}, \ldots, T_{q, x_{m}}$ pairwise commute. When expanding the left-hand side of (4.4), it is clear that the resulting terms can be parametrized by matrices in $M_{1}^{(m)}$ : the term corresponding to $\tau \in M_{1}^{(m)}$ is the product of $(-1)^{k} T_{q, x_{i}}^{k} T_{q, x_{j}}^{1-k}$, where $k \in\{0,1\}$ ranges over the elements of $\tau$ strictly above the main diagonal. Thus the term corresponding to $\tau$ is

$$
\prod_{1 \leq i<j \leq m}(-1)^{\tau_{i, j}} T_{q, x_{i}}^{\tau_{i, j}} T_{q, x_{j}}^{1-\tau_{i, j}}=(-1)^{\sum_{1 \leq i<j \leq m} \tau_{i, j}} \prod_{1 \leq i<j \leq m} T_{q, x_{i}}^{\tau_{i, j}} T_{q, x_{j}}^{1-\tau_{i, j}} .
$$

We are left to show

$$
\prod_{1 \leq i<j \leq m} T_{q, x_{i}}^{\tau_{i, j}} T_{q, x_{j}}^{1-\tau_{i, j}}=\prod_{k=1}^{m} T_{q, x_{k}}^{k-1+\tau_{k}^{+}-\tau_{k}^{-}} .
$$

Both sides of (4.5) are of the form $T_{q, x_{1}}^{p_{1}} \cdots T_{q, x_{m}}^{p_{m}}$, for some $p_{1}, \ldots, p_{m} \in \mathbb{Z}_{\geq 0}$, so we simply need to check the equality between exponents $p_{k}$ of $T_{q, x_{k}}$ in both sides, for an arbitrary $1 \leq k \leq m$. In the left side, there are $k-1$ factors of the form $T_{q, x_{i}}^{\tau_{i, k}} T_{q, x_{k}}^{1-\tau_{i, k}}, i \leq k-1$, which overall contribute $k-1-\sum_{i=1}^{k-1} \tau_{i, k}=k-1-\tau_{k}^{-}$to the exponent of $T_{q, x_{k}}$. Moreover there are $m-k$ factors of the form $T_{q, x_{k}}^{\tau_{k, j}} T_{q, x_{j}}^{1-\tau_{k, j}}, k+1 \leq i$, which contribute $\sum_{i=k+1}^{m} \tau_{k, i}=\tau_{k}^{+}$to the exponent of $T_{q, x_{k}}$. Therefore the power of $T_{q, x_{k}}$ in the left-hand side of (4.5) is $T_{q, x_{k}}^{k-1+\tau_{k}^{+}-\tau_{k}^{-}}$. Evidently, the power of $T_{q, x_{k}}$ in the right-hand side of (4.5) if also $T_{q, x_{k}}^{k-1+\tau_{k}^{+}-\tau_{k}^{-}}$, which finishes the proof.

Example 4.4. We discuss the first nontrivial example of the multiplicative formula for Macdonald polynomials (an example that is not dealt with in the Corollaries above): $\theta=2, m=3$. The formula in this case is

$$
\begin{aligned}
P_{\lambda}\left(x_{1}, x_{2}, x_{3} ; N, q, q^{2}\right)= & \frac{q^{25-12 N}}{\left([2 N-1]_{q}\right)^{2}\left([2 N-2]_{q}\right)^{2}[2 N-3]_{q}[2 N-4]_{q}} \\
& \times \frac{1}{\prod_{j=-1}^{2 N-7}\left(x_{1}-q^{j}\right)\left(x_{2}-q^{j}\right)\left(x_{3}-q^{j}\right)} \frac{1}{\prod_{1 \leq i<j \leq 3}\left(x_{i}-x_{j}\right)\left(x_{i}-q x_{j}\right)} \\
& \times \widehat{\mathcal{D}}_{q, 2}^{(3)}\left\{\prod_{i=1}^{3} P_{\lambda}\left(x_{i} ; N, q, q^{2}\right) \prod_{j=-1}^{2 N-3}\left(x_{i}-q^{j}\right)\right\},
\end{aligned}
$$

the $q$-difference operator is

$$
\begin{aligned}
\widehat{\mathcal{D}}_{q, 2}^{(3)}=\frac{1}{(q-1)^{6}} \sum_{\substack{a, b, c \in \mathbb{Z} \\
0 \leq a, b, c \leq 2}}\left\{C_{a}^{\left(q, q^{2}\right)}\left(x_{2}^{-1} x_{1} q^{-2+b-c}\right)\right. \\
\left.\times C_{b, c}^{\left(q, q^{2}\right)}\left(x_{3}^{-1} x_{1} q^{-2}, x_{3}^{-1} x_{2} q^{-2}\right) T_{q, x_{1}}^{a+b} T_{q, x_{2}}^{2+c-a} T_{q, x_{3}}^{4-b-c}\right\} .
\end{aligned}
$$


We can also write the $q$-difference operator in terms of the $q$-degree operators $\left\{D_{q, x_{i}}: i=1,2,3\right\}$ by using $\frac{1}{q-1} T_{q, x_{i}}=\frac{1}{q-1}+D_{q, x_{i}}$. Then we can replace the operator $\widehat{\mathcal{D}}_{q, 2}^{(3)}$ with the sum of operators $\mathcal{D}_{q, 2}^{(3, \text { top })}+\mathcal{A}_{q, 2}^{(3)}$, where

$$
\begin{gathered}
\mathcal{D}_{q, 2}^{(3, \text { top })}=\sum_{\substack{a, b, c \in \mathbb{Z} \\
0 \leq a, b, c \leq 2}}\left\{C_{a}^{\left(q, q^{2}\right)}\left(x_{2}^{-1} x_{1} q^{-2+b-c}\right)\right. \\
\left.\times C_{b, c}^{\left(q, q^{2}\right)}\left(x_{3}^{-1} x_{1} q^{-2}, x_{3}^{-1} x_{2} q^{-2}\right) D_{q, x_{1}}^{a+b} D_{q, x_{2}}^{2+c-a} D_{q, x_{3}}^{4-b-c}\right\}, \\
\mathcal{A}_{q, 2}^{(3)}=\sum_{\substack{i_{1}, i_{2}, i_{3} \geq 0 \\
i_{1}+i_{2}+i_{3} \leq 5}} \frac{f_{i_{1}, i_{2}, i_{3}}\left(x_{1}, x_{2}, x_{3} ; q, 2\right)}{(q-1)^{6-i_{1}-i_{2}-i_{3}}} D_{q, x_{1}}^{i_{1}} D_{q, x_{2}}^{i_{2}} D_{q, x_{3}}^{i_{3}},
\end{gathered}
$$

and $f_{i_{1}, i_{2}, i_{3}}\left(x_{1}, x_{2}, x_{3} ; q, 2\right)$ are certain rational functions. With the help of Sage, we found

$$
f_{i_{1}, i_{2}, i_{3}}\left(x_{1}, x_{2}, x_{3} ; q, 2\right)=0, \quad \text { for all } i_{1}+i_{2}+i_{3} \leq 2 .
$$

There are nontrivial rational functions $f_{i_{1}, i_{2}, i_{3}}\left(x_{1}, x_{2}, x_{3} ; q, 2\right)$ as well, for some $i_{1}+i_{2}+i_{3} \geq 3$, e.g.,

$$
\begin{aligned}
& f_{4,1,0}\left(x_{1}, x_{2}, x_{3} ; q, 2\right)=-(q-1) \frac{\left(x_{2}+x_{3}\right)\left(x_{1}-q x_{3}\right)\left(x_{1}-q x_{2}\right)}{\left(q x_{2}-x_{3}\right)\left(q x_{1}-x_{3}\right)\left(q x_{1}-x_{2}\right)} \\
& f_{2,1,1}\left(x_{1}, x_{2}, x_{3} ; q, 2\right)=-(q-1)^{2} \frac{(q+1)\left(x_{2}-x_{3}\right)\left(x_{1}^{2}+x_{2} x_{3}+2 x_{1} x_{2}+2 x_{1} x_{3}\right)}{\left(q x_{1}-x_{2}\right)\left(q x_{1}-x_{3}\right)\left(q x_{2}-x_{3}\right)} .
\end{aligned}
$$

Two important observations are in order. First, from Corollaries 4.2 and 4.3, one might believe that $\mathcal{D}_{q, \theta}^{(m)}$ is, in general, homogeneous of degree $\theta\left(\begin{array}{c}m \\ 2\end{array}\right)$ as a functions of the operators $\left\{D_{q, x_{i}}: i=1,2,3\right\}$. However, this example disproves it. Second, the terms $f_{i_{1}, i_{2}, i_{3}}\left(x_{1}, x_{2}, x_{3} ; q, 2\right)$ above make us suspect that $f_{i_{1}, i_{2}, i_{3}}\left(x_{1}, x_{2}, x_{3} ; q, 2\right)$ is divisible by $(q-1)^{6-i_{1}-i_{2}-i_{3}} \forall 0 \leq i_{1}+i_{2}+i_{3} \leq 5$. We have checked this fact in the computer. In fact, we believe that the analogous statement for general $m, \theta \in \mathbb{N}$ holds true, but the author could not prove it.

\subsection{Proof of Theorem 4.1}

Fix a positive signature $\lambda \in \mathbb{G}_{N}^{+}$and let us prove equation (4.1); we extend the result for all signatures $\lambda \in \mathbb{G}_{N}$ at the end.

Let us consider $m$ positive integers $n_{1}>n_{2}>\cdots>n_{m}>\theta(N+m)$. By the index-argument symmetry, Theorem 2.2 , applied to $\lambda \in \mathbb{G T}_{N}^{+}$and $\mu=\left(n_{1} \geq \cdots \geq n_{m} \geq 0 \geq \cdots \geq 0\right) \in \mathbb{G T}_{N}^{+}$, as well as the definition of the dual Macdonald polynomials $Q_{\mu}(\cdot ; q, t)$, we obtain

$$
\begin{gathered}
\frac{P_{\lambda}\left(q^{n_{1}} t^{N-1}, \ldots, q^{n_{m}} t^{N-m}, t^{N-m-1}, \ldots, t, 1 ; q, t\right)}{P_{\lambda}\left(t^{N-1}, t^{N-2}, \ldots, 1 ; q, t\right)} \\
=\frac{Q_{\left(n_{1}, \ldots, n_{m}\right)}\left(q^{\lambda_{1}} t^{N-1}, q^{\lambda_{2}} t^{N-2}, \ldots, q^{\lambda_{N}} ; q, t\right)}{Q_{\left(n_{1}, \ldots, n_{m}\right)}\left(t^{N-1}, t^{N-2}, \ldots, 1 ; q, t\right)} .
\end{gathered}
$$

Apply the Jacobi-Trudi formula for Macdonald polynomials, Theorem 2.7, to the numerator of (4.8), then multiply and divide the term parametrized by $\tau$ by the product

$$
\prod_{s=1}^{m} g_{n_{s}+\tau_{s}^{+}-\tau_{s}^{-}}\left(t^{N-1}, \ldots, t, 1 ; q, t\right),
$$


so (4.8) equals

$$
\begin{aligned}
& \sum_{\tau \in M^{(m)}}\left\{\prod_{s=1}^{m-1} C_{\tau_{1, s+1}, \ldots, \tau_{s, s+1}}^{(q, t)}\left(\left\{u_{i}=q^{n_{i}-n_{s+1}+\sum_{j=s+2}^{m}\left(\tau_{i, j}-\tau_{s+1, j}\right)} t^{s-i}: 1 \leq i \leq s\right\}\right)\right. \\
&\left.\quad \times \prod_{s=1}^{m} \frac{g_{n_{s}+\tau_{s}^{+}-\tau_{s}^{-}}\left(q^{\lambda_{1}} t^{N-1}, \ldots, q^{\lambda_{N}} ; q, t\right)}{g_{n_{s}+\tau_{s}^{+}-\tau_{s}^{-}}\left(t^{N-1}, \ldots, t, 1 ; q, t\right)} \times \frac{\prod_{s=1}^{m} g_{n_{s}+\tau_{s}^{+}-\tau_{s}^{-}}\left(t^{N-1}, \ldots, t, 1 ; q, t\right)}{Q_{\left(n_{1}, \ldots, n_{m}\right)}\left(t^{N-1}, \ldots, t, 1 ; q, t\right)}\right\} .
\end{aligned}
$$

(In equation (4.9), we are setting $g_{n}(q, t)=0$ if $n$ is a nonpositive integer.) Recall that $t=q^{\theta}$, $\theta \in \mathbb{N}$. In view of Lemma B.1, the only terms in the sum (4.9) with nonzero contributions are those parametrized by $m \times m$ matrices whose entries belong to the set $\{0,1, \ldots, \theta\}$; define $M_{\theta}^{(m)}$ to be the set of such matrices. Notice that $n_{s}+\tau_{s}^{+}-\tau_{s}^{-}>0$ for all $\tau \in M_{\theta}^{(m)}$, because of our initial assumption on the values of $n_{1}, \ldots, n_{m}$. By another application of the index-argument symmetry (and of the identity (2.4) above), we have

$$
\begin{aligned}
& \frac{g_{n_{s}+\tau_{s}^{+}-\tau_{s}^{+}}\left(q^{\lambda_{1}} t^{N-1}, \ldots, q^{\lambda_{N}} ; q, t\right)}{g_{n_{s}+\tau_{s}^{+}-\tau_{s}^{-}}\left(t^{N-1}, \ldots, t, 1 ; q, t\right)} \\
& \quad=\frac{P_{\lambda}\left(q^{n_{s}+\tau_{s}^{+}-\tau_{s}^{-}} t^{N-1}, t^{N-2}, \ldots, t, 1 ; q, t\right)}{P_{\lambda}\left(t^{N-1}, \ldots, t, 1 ; q, t\right)} \quad \forall 1 \leq s \leq m .
\end{aligned}
$$

Plugging (4.2) into (4.9), we obtain

$$
\begin{aligned}
& \frac{P_{\lambda}\left(q^{n_{1}+\theta(N-1)}, \ldots, q^{n_{m}+\theta(N-m)}, t^{N-m-1}, \ldots, t, 1 ; q, t\right)}{P_{\lambda}\left(t^{N-1}, t^{N-2}, \ldots, 1 ; q, t\right)} \\
& =\sum_{\tau \in M_{\theta}^{(m)}}\left\{\prod_{s=1}^{m-1} C_{\tau_{1, s+1}, \ldots, \tau_{s, s+1}}^{\left(q, q^{\theta}\right)}\left(\left\{u_{i}=q^{n_{i}-n_{s+1}+\sum_{j=s+2}^{m}\left(\tau_{i, j}-\tau_{s+1, j}\right)+\theta(s-i)}: 1 \leq i \leq s\right\}\right)\right. \\
& \left.\quad \times \frac{\prod_{s=1}^{m} g_{n_{s}+\tau_{s}^{+}-\tau_{s}^{-}}\left(t^{N-1}, \ldots, t, 1 ; q, t\right)}{Q_{\left(n_{1}, \ldots, n_{m}\right)}\left(t^{N-1}, \ldots, t, 1 ; q, t\right)} \times \prod_{s=1}^{m} \frac{P_{\lambda}\left(q^{n_{s}+\tau_{s}^{+}-\tau_{s}^{-}+\theta(N-1)}, t^{N-2}, \ldots, t, 1 ; q, t\right)}{P_{\lambda}\left(t^{N-1}, \ldots, t, 1 ; q, t\right)}\right\} .
\end{aligned}
$$

Let us make the change of variables

$$
z_{s} \stackrel{\text { def }}{=} q^{n_{s}+\theta(N-s)}, \quad 1 \leq s \leq m,
$$

and rewrite some terms from (4.11) in these new variables. Clearly the left-hand side of equality (4.11) is the Macdonald character $P_{\lambda}\left(z_{1}, \ldots, z_{m} ; N, q, q^{\theta}\right)$. It is also evident that the variable $u_{i}$ in the term $C_{\tau_{1, s+1}, \ldots, \tau_{s, s+1}}^{\left(q, q^{\theta}\right)}$ can be rewritten as $u_{i}=z_{s+1}^{-1} z_{i} q^{-\theta+\sum_{j=s+2}^{m}\left(\tau_{i, j}-\tau_{s+1, j}\right)}$, for $1 \leq i \leq s$. Additionally we have $q^{n_{s}+\tau_{s}^{+}-\tau_{s}^{-}+\theta(N-1)}=T_{q, z_{s}}^{\theta s-\theta+\tau_{s}^{+}-\tau_{s}^{-}}\left(z_{s}\right)$, for all $s$, which implies that the last product in (4.11) can be written as

$$
\prod_{s=1}^{m} T_{q, x_{s}}^{\theta(s-1)+\tau_{s}^{+}-\tau_{s}^{-}}\left(\prod_{s=1}^{m} P_{\lambda}\left(z_{s} ; N, q, q^{\theta}\right)\right) .
$$

Next, we need to rewrite

$$
\frac{\prod_{s=1}^{m} g_{n_{s}+\tau_{s}^{+}-\tau_{s}^{-}}\left(t^{N-1}, \ldots, t, 1 ; q, t\right)}{Q_{\left(n_{1}, \ldots, n_{m}\right)}\left(t^{N-1}, \ldots, t, 1 ; q, t\right)}
$$


in terms of $z_{1}, \ldots, z_{m}$. From Corollary 2.4 of the evaluation identity for Macdonald polynomials, we obtain

$$
\begin{aligned}
g_{p}\left(t^{N-1}, t^{N-2}, \ldots, t, 1 ; q, t\right) & =\prod_{s=(1, j) \in(p)} \frac{1-q^{a^{\prime}(s)+\theta\left(N-l^{\prime}(s)\right)}}{1-q^{a(s)+1+\theta l(s)}} \\
= & \prod_{i=1}^{p} \frac{1-q^{i-1+\theta N}}{1-q^{p-i+1}}=\prod_{j=1}^{\theta N-1} \frac{1-q^{p+j}}{1-q^{j}}=\frac{1}{(1-q)^{\theta N-1}} \frac{\prod_{j=1}^{N-1}\left(1-q^{p+j}\right)}{[\theta N-1]_{q} !}
\end{aligned}
$$

for any $p \in \mathbb{N}, p>\theta N$. By similar, but more complicated, computations we find

$$
\begin{aligned}
Q_{\left(p_{1}, \ldots, p_{m}\right)}\left(t^{N-1}, \ldots, t, 1 ; q, t\right) & \frac{1}{(1-q)^{m(\theta N-1)-\theta m(m-1) / 2}} \times \prod_{s=1}^{\theta} \prod_{1 \leq i<j \leq m} \frac{q^{p_{j}}-q^{p_{i}+s+\theta(j-i-1)}}{1-q^{p_{i}+s+\theta(j-i-1)}} \\
& \\
& \times \frac{\prod_{j=1}^{\theta(N-m+1)-1}\left(1-q^{p_{m}+j}\right) \cdot \prod_{j=1}^{\theta(N-m+2)-1}\left(1-q^{p_{m-1}+j}\right) \cdots \prod_{j=1}^{\theta N-1}\left(1-q^{p_{1}+j}\right)}{[\theta(N-m+1)-1]_{q} ![\theta(N-m+2)-1]_{q} ! \cdots[\theta N-1]_{q} !},
\end{aligned}
$$

for any partition $\left(p_{1}>p_{2}>\cdots>p_{m}>0\right)$ with $p_{i}>\theta(N-i+1)$ for all $1 \leq i \leq m$. From (4.13) and (4.14), we obtain

$$
\begin{aligned}
& \frac{\prod_{s=1}^{m} g_{n_{s}+\tau_{s}^{+}-\tau_{s}^{-}}\left(t^{N-1}, \ldots, t, 1\right)}{Q_{\left(n_{1}, \ldots, n_{m}\right)}\left(t^{N-1}, \ldots, t, 1 ; q, t\right)}=\frac{1}{(1-q)^{\theta m(m-1) / 2}} \prod_{i=1}^{m} \frac{[\theta(N-i+1)-1]_{q} !}{[\theta N-1]_{q} !} \\
& \times \frac{\prod_{j=1}^{\theta N-1}\left(1-q^{n_{1}+\tau_{1}^{+}-\tau_{1}^{-}+j}\right)}{\prod_{j=1}^{\theta-1}\left(1-q^{n_{1}+j}\right)} \cdot \frac{\prod_{j=1}^{\theta N-1}\left(1-q^{n_{2}+\tau_{2}^{+}-\tau_{2}^{-}+j}\right)}{\prod_{j=1}^{\theta(N-1)-1}\left(1-q^{n_{2}+j}\right)} \cdot \frac{\prod_{j=1}^{\theta N-1}\left(1-q^{n_{m}+\tau_{m}^{+}-\tau_{m}^{-}+j}\right)}{\prod_{j=1}^{\theta(N-m+1)-1}\left(1-q^{n_{m}+j}\right)} \\
& \times \prod_{s=1}^{\theta} \prod_{1 \leq i<j \leq m} \frac{1-q^{n_{i}+s+\theta(j-i-1)}}{q^{n_{j}}-q^{n_{i}+s+\theta(j-i-1)}} .
\end{aligned}
$$

Observe that we used our assumption $n_{1}>n_{2}>\cdots>n_{m}>\theta(N+m)$ to guarantee that (4.13) and (4.14) are applicable. We have to rewrite both (4.16) and (4.17) in terms of $z_{s}$. Let us begin with (4.16), which is a product of $m$ terms; for $1 \leq r \leq m$, the $r^{\text {th }}$ term is

$$
\begin{aligned}
& \frac{\prod_{j=1}^{\theta N-1}\left(1-q^{n_{r}+\tau_{r}^{+}-\tau_{r}^{-}+j}\right)}{\prod_{j=1}^{\theta(N-r+1)-1}\left(1-q^{n_{r}+j}\right)}=\frac{\prod_{j=1}^{\theta N-1}\left(1-q^{-\theta(N-r)} z_{r} q^{\tau_{r}^{+}-\tau_{r}^{-}+j}\right)}{\prod_{j=1}^{\theta(N-r+1)-1}\left(1-q^{-\theta(N-r)} z_{r} q^{j}\right)} \\
& =(-1)^{\theta(r-1)} q^{-\sum_{j=\theta(N-r+1)}^{\theta N-1}(\theta(N-r)-j)} \cdot \frac{\prod_{j=1}^{\theta N-1}\left(z_{r} q^{\tau_{r}^{+}-\tau_{r}^{+}}-q^{\theta(N-r)-j}\right)}{\prod_{j=1}^{\theta(N-r+1)-1}\left(z_{r}-q^{\theta(N-r)-j}\right)} \\
& =(-1)^{\theta(r-1)} q^{-\sum_{j=\theta(N-r+1)}^{\theta N-1}(\theta(N-r)-j)} q^{-(\theta r-\theta)(\theta N-1)} \frac{\prod_{j=1}^{\theta N-1}\left(z_{r} q^{\theta r-\theta+\tau_{r}^{+}-\tau_{r}^{-}}-q^{\theta(N-1)-j}\right)}{\prod_{j=1}^{\theta(N-r+1)-1}\left(z_{r}-q^{\theta(N-r)-j}\right)} .
\end{aligned}
$$


Under the change of indexing $j \mapsto \theta(N-r+1)-j$, the product in the denominator of (4.18) becomes

$$
\prod_{j=1}^{\theta(N-r+1)-1}\left(z_{r}-q^{\theta(N-r)-j}\right)=\prod_{j=1}^{\theta(N-r+1)-1}\left(z_{r}-q^{j-\theta}\right)
$$

whereas the numerator of (4.18) can be expressed as

$$
\prod_{j=1}^{\theta N-1}\left(z_{r} q^{\theta r-\theta+\tau_{r}^{+}-\tau_{r}^{-}}-q^{\theta(N-1)-j}\right)=T_{q, z_{r}}^{\theta r-\theta+\tau_{r}^{+}-\tau_{r}^{-}} \prod_{j=1}^{\theta N-1}\left(z_{r}-q^{\theta(N-1)-j}\right) .
$$

Define also

$$
c(N, m, \theta)=-\sum_{r=1}^{m} \sum_{j=\theta(N-r+1)}^{\theta N-1}(\theta(N-r)-j)-\sum_{r=1}^{m}(\theta r-\theta)(\theta N-1),
$$

which is the power of $q$ coming from the terms (4.18), for $1 \leq r \leq m$. Thus (4.16) equals

$$
\frac{(-1)^{\theta m(m-1) / 2} q^{c(N, m, \theta)}}{\prod_{r=1}^{m} \prod_{j=1}^{\theta(N-r+1)-1}\left(z_{r}-q^{j-\theta}\right)} \cdot \prod_{r=1}^{m} T_{q, z_{r}}^{\theta r-\theta+\tau_{r}^{+}-\tau_{r}^{-}}\left(\prod_{j=1}^{\theta N-1}\left(z_{r}-q^{\theta(N-1)-j}\right)\right) .
$$

On the other hand, (4.17) can be expressed in terms of the variables $z_{i}$ as follows:

$$
\begin{aligned}
& \prod_{r=1}^{\theta} \prod_{i<j} \frac{1-q^{n_{i}+r+\theta(j-i-1)}}{q^{n_{j}}-q^{n_{i}+r+\theta(j-i-1)}}=\prod_{r=1}^{\theta} \prod_{i<j} \frac{q^{n_{i}+r+\theta(j-i-1)}-1}{q^{n_{i}+r+\theta(j-i-1)}-q^{n_{j}}} \\
& \quad=\prod_{r=1}^{\theta} \prod_{i<j} \frac{q^{n_{i}+r+\theta(N-i-1)}-q^{\theta(N-j)}}{q^{n_{i}+r+\theta(N-i-1)}-q^{n_{j}+\theta(N-j)}}=\prod_{r=1}^{\theta} \prod_{i<j} \frac{z_{i} q^{r-\theta}-q^{\theta(N-j)}}{z_{i} q^{r-\theta}-z_{j}} \\
& \quad=\prod_{r=1}^{\theta} \prod_{i<j} \frac{z_{i}-q^{\theta(N-j+1)-r}}{z_{i}-z_{j} q^{\theta-r}} .
\end{aligned}
$$

The denominator of (4.21) is $\prod_{\substack{1 \leq i<j \leq m \\ 0 \leq k<\theta}}\left(z_{i}-q^{k} z_{j}\right)$, and the numerator is $\prod_{r=1}^{m} \prod_{j=\theta(N-m+1)}^{\theta(N-i+1)-1}\left(z_{r}-q^{j-\theta}\right)$.

Then (4.17) equals

$$
\frac{\prod_{r=1}^{m} \prod_{\substack{1 \leq i<j \leq m \\ 0 \leq k<\theta}}^{\theta(N-r+1)-1}\left(z_{i}-q^{k} z_{j}\right)}{\left.\prod_{r}-q^{j-\theta}\right)} .
$$

Therefore after the change of variables (4.12), and using (4.15), (4.16), (4.17), (4.20), (4.22) and the identities after (4.12), equation (4.11) yields the desired (4.1) for any $x_{s}=q^{n_{s}+\theta(N-s)}$, $1 \leq s \leq m$, such that $n_{1}>\cdots>n_{m}>\theta(N+m)$, provided the equality

$$
c(N, m, \theta)=\theta^{2}\left(\begin{array}{c}
m+1 \\
3
\end{array}\right)-\left\{N \theta^{2}-\left(\begin{array}{c}
\theta+1 \\
2
\end{array}\right)\right\}\left(\begin{array}{c}
m \\
2
\end{array}\right)
$$

holds, where $c(N, m, \theta)$ is defined in (4.19). This is an easy exercise. 
We have just proved that the statement of Theorem 4.1 holds for all $x_{s}=q^{n_{s}+\theta(N-s)}$, for all $n_{1}>n_{2}>\cdots>n_{m}>\theta(N+m)$. Since both sides of the equality (4.1) are evidently rational functions in $x_{1}, \ldots, x_{m}$, an easy algebro-geometric argument shows the equality holds for all $x_{1}, \ldots, x_{m} \in \mathbb{C}$, as desired.

We still have to extend the theorem to all signatures. Let us prove (4.1) for an arbitrary $\lambda \in \mathbb{G T}_{N}$. If $\lambda \in \mathbb{G T}_{N}^{+}$, then we are done. Otherwise, choose any $p \in \mathbb{N}$ large enough so that $\widetilde{\lambda} \stackrel{\text { def }}{=}\left(\lambda_{1}+p, \lambda_{2}+p, \ldots, \lambda_{N}+p\right) \in \mathbb{G T}_{N}^{+}$. By homogeneity of Macdonald polynomials, we have

$$
P_{\widetilde{\lambda}}\left(x_{1}, \ldots, x_{m} ; N, q, q^{\theta}\right)=P_{\lambda}\left(x_{1}, \ldots, x_{m} ; N, q, q^{\theta}\right)\left(x_{1} \cdots x_{m}\right)^{p} q^{-\theta p N m} q^{\theta p m(m+1) / 2}
$$

and

$$
\begin{aligned}
P_{\widetilde{\lambda}}\left(x_{i} ; N, q, q^{\theta}\right)=P_{\lambda}\left(x_{i} ; N, q, q^{\theta}\right) x_{i}^{p} q^{-\theta p N} q^{\theta p} \quad \forall 1 \leq i \leq m & \\
\Rightarrow T_{q, x_{i}}^{(i-1) \theta+\tau_{i}^{+}-\tau_{i}^{-}} P_{\widetilde{\lambda}}\left(x_{i} ; N, q, q^{\theta}\right)= & q^{-\theta p N} q^{p\left(i \theta+\tau_{i}^{+}-\tau_{i}^{-}\right)} x_{i}^{p} \\
& \times T_{q, x_{i}}^{(i-1) \theta+\tau_{i}^{+}-\tau_{i}^{-}} P_{\lambda}\left(x_{i} ; N, q, q^{\theta}\right) \quad \forall 1 \leq i \leq m .
\end{aligned}
$$

We know that (4.1) holds for $\widetilde{\lambda}$; from the expressions above, it holds also for $\lambda$ provided that (the powers of $q$ match)

$$
-\theta p N m+\frac{\theta p m(m+1)}{2}=\sum_{i=1}^{m}\left(-\theta p N+p\left(i \theta+\tau_{i}^{+}-\tau_{i}^{-}\right)\right) .
$$

The latter equation is easy to check, and the proof of Theorem 4.1 is therefore finished.

\section{$5 \quad$ Asymptotics of Macdonald characters}

In the remaining of the paper, starting here, we denote the Macdonald polynomial $P_{\lambda}\left(x_{1}, \ldots, x_{N}\right.$; $q, t)$ simply by $P_{\lambda}\left(x_{1}, \ldots, x_{N}\right)$.

In this section, assume that $\theta \in \mathbb{N}$ and let $t=q^{\theta}$. We study the asymptotics of (certain normalization of) Macdonald characters of a fixed number $m$ of variables, as the rank $N$ tends to infinity and the signatures $\lambda(N)$ stabilize in certain way that we define next.

Definition 5.1. Let

$$
\mathcal{N} \stackrel{\text { def }}{=}\left\{\nu=\left(\nu_{1}, \nu_{2}, \ldots\right) \in \mathbb{Z}^{\infty}: \nu_{1} \leq \nu_{2} \leq \ldots\right\}
$$

be the set of weakly increasing integer sequences. We say that the sequence $\{\lambda(N)\}_{N \geq 1}$ of signatures, $\lambda(N) \in \mathbb{G T}_{N}$, stabilizes to $\nu \in \mathcal{N}$ if we have the following limits

$$
\lim _{N \rightarrow \infty} \lambda_{N-i+1}(N)=\nu_{i}, \quad \forall i=1,2, \ldots
$$

In other words $\{\lambda(N)\}_{N \geq 1}$ stabilizes to $\nu$ if there exists a sequence of integers $0<N_{1}<N_{2}<\cdots$ such that $\lambda_{N-i+1}(N)=\nu_{i}, \forall N>N_{i}, i=1,2, \ldots$

\subsection{Asymptotics of Macdonald characters of one variable}

Theorem 5.2. Let $t=q^{\theta}, \theta \in \mathbb{N}$, and $\{\lambda(N)\}_{N \geq 1}, \lambda(N) \in \mathbb{G}_{N}$, be a sequence of signatures that stabilizes to $\nu \in \mathcal{N}$. Then

$$
\lim _{N \rightarrow \infty} \frac{P_{\lambda(N)}\left(x, t^{-1}, \ldots, t^{1-N}\right)}{P_{\lambda(N)}\left(1, t^{-1}, t^{-2}, \ldots, t^{1-N}\right)}=\Phi^{\nu}(x ; q, t),
$$


where

$$
\Phi^{\nu}(x ; q, t) \stackrel{\text { def }}{=} \frac{(q ; q)_{\infty}}{(x q ; q)_{\infty}} \frac{\ln q}{2 \pi \sqrt{-1}} \int_{\mathcal{C}^{+}} x^{z} \prod_{i=1}^{\infty} \frac{\left(q^{-z+\nu_{i}} t^{i} ; q\right)_{\infty}}{\left(q^{-z+\nu_{i}} t^{i-1} ; q\right)_{\infty}} \mathrm{d} z,
$$

and the contour $\mathcal{C}^{+}$is the infinite positive contour consisting of $\left[-M+\frac{\pi \sqrt{-1}}{\ln q},-M-\frac{\pi \sqrt{-1}}{\ln q}\right]$ and the lines $\left[-M+\frac{\pi \sqrt{-1}}{\ln q},+\infty+\frac{\pi \sqrt{-1}}{\ln q}\right),\left[-M-\frac{\pi \sqrt{-1}}{\ln q},+\infty-\frac{\pi \sqrt{-1}}{\ln q}\right)$ for an arbitrary $M>\max \left\{0,-\nu_{1}\right\}$, see Fig. 2. The function $\Phi^{\nu}(x ; q, t)$ is defined by the formula above in the domain

$$
\mathcal{U} \stackrel{\text { def }}{=} \bigcap_{k \geq 1}\left\{x \neq q^{-k}\right\} \cap\{x \neq 0\},
$$

and admits an analytic continuation to the domain $\{x \neq 0\}=\mathbb{C} \backslash\{0\}$. Moreover if $\nu_{1} \geq 0$, the function $\Phi^{\nu}(x ; q, t)$ can be analytically continued to $\mathbb{C}$.

The convergence (5.1) is uniform on compact subsets of $\mathbb{C} \backslash\{0\}$, and if $\nu_{1} \geq 0$, then it is uniform on compact subsets of $\mathbb{C}$.

Remark 5.3. The theorem is a direct application of the integral representation in Theorem 3.1. If we use instead Theorems 3.2 and 3.3, we could possibly extend the theorem for general $q, t \in(0,1)$. Since our main application does require $\theta \in \mathbb{N}$ and $t=q^{\theta}$, we do not bother to pursue the more general case $q, t \in(0,1)$.

Proof. Let us first prove that the limit (5.1) holds uniformly for $x$ in compact subsets of $\mathcal{U}$. We use the integral representation for Macdonald characters of one variable, Theorem 3.1, for the signature $\lambda(N) \in \mathbb{G}_{N}$, and with $x$ replaced by $x t^{N}, x \in \mathcal{U}$. Since $P_{\lambda(N)}$ is a homogeneous polynomial of degree $|\lambda(N)|$, then the left-hand side is

$$
t^{|\lambda(N)|} \frac{P_{\lambda(N)}\left(x, t^{-1}, t^{-2}, \ldots, t^{1-N}\right)}{P_{\lambda(N)}\left(1, t^{-1}, t^{-2}, \ldots, t^{1-N}\right)} .
$$

After simple algebraic manipulations, the right-hand side becomes

$$
q^{-\left(\begin{array}{c}
\theta N \\
2
\end{array}\right)} \frac{(q ; q)_{\theta N-1}}{(x q ; q)_{\theta N-1}} \frac{\ln q}{2 \pi \sqrt{-1}} \oint_{\mathcal{C}_{0}} \frac{x^{z}}{\prod_{i=1}^{N} \prod_{j=0}^{\theta-1}\left(q^{-\left(\lambda_{i}(N)+\theta(N-i)+j\right)}-q^{-z}\right)} \mathrm{d} z .
$$

Therefore we conclude (make change of variables $i \mapsto N-i+1$ in the inner product)

$$
\begin{aligned}
& \frac{P_{\lambda(N)}\left(x, t^{-1}, t^{-2}, \ldots, t^{1-N}\right)}{P_{\lambda(N)}\left(1, t^{-1}, t^{-2}, \ldots, t^{1-N}\right)} \\
& \quad=\frac{(q ; q)_{\theta N-1}}{(x q ; q)_{\theta N-1}} \frac{\ln q}{2 \pi \sqrt{-1}} \oint_{\mathcal{C}_{0}} \frac{x^{z}}{\prod_{i=1}^{N} \prod_{j=0}^{\theta-1}\left(1-q^{-z} q^{\lambda_{N-i+1}(N)+\theta(i-1)+j}\right)} \mathrm{d} z
\end{aligned}
$$

where $\mathcal{C}_{0}$ is a finite contour encloses all real poles of the integrand and no other poles. We have the limit

$$
\lim _{N \rightarrow \infty} \frac{(q ; q)_{\theta N-1}}{(x q ; q)_{\theta N-1}}=\frac{(q ; q)_{\infty}}{(x q ; q)_{\infty}}
$$

uniformly for $x$ belonging to compact subsets of $\mathcal{U}$.

Next modify the contour $\mathcal{C}_{0}$ into an infinite contour $\mathcal{C}^{+}$as described in the statement of the theorem. This is possible to do because all the poles of the integrands of (5.3) belong to the 
interior of $\mathcal{C}^{+}$, as $N$ grows. Moreover the resulting integral is well-posed for large enough $N$ since the integrand is of order $\left(|x| q^{\theta N}\right)^{\Re z}$ and for any compact set $K \subset \mathbb{C}$, there exists $N_{0} \in \mathbb{N}$ such that $\sup _{N>N_{0}} \sup _{x \in K}\left\{|x| q^{\theta N}\right\}<1$.

We now look at the asymptotics of the integral in $(5.3)$, with $\mathcal{C}_{0}$ replaced by $\mathcal{C}^{+}$. The denominator in the integrand has the following limit

$$
\begin{aligned}
\lim _{N \rightarrow \infty} & \prod_{i=1}^{N} \prod_{j=0}^{\theta-1}\left(1-q^{-z+\lambda_{N-i+1}(N)+\theta(i-1)+j}\right) \\
& =\prod_{i=1}^{\infty} \prod_{j=0}^{\theta-1}\left(1-q^{-z+\nu_{i}+\theta(i-1)+j}\right)=\prod_{i=1}^{\infty} \frac{\left(q^{-z+\nu_{i}} t^{i-1} ; q\right)_{\infty}}{\left(q^{-z+\nu_{i}} t^{i} ; q\right)_{\infty}} .
\end{aligned}
$$

The limit above can be justified properly by using the dominated convergence theorem and the estimate

$$
\begin{aligned}
& \sum_{i=1}^{N} \sum_{j=0}^{\theta-1}\left|q^{-z+\lambda_{N-i+1}(N)+\theta(i-1)+j}\right| \\
& \quad \leq \sum_{i=1}^{N} \sum_{j=0}^{\theta-1} q^{-\Re z+\lambda_{N}(N)+\theta(i-1)+j} \leq c \sum_{i=1}^{N} \sum_{j=0}^{\theta-1} q^{\theta(i-1)+j}<\frac{c}{1-q},
\end{aligned}
$$

valid for some constant $c>0$ such that $q^{-\Re z+\lambda_{N}(N)}<c$ for all $N \geq 1$ (it exists because $\left.\lim _{N \rightarrow \infty} \lambda_{N}(N)=\nu_{1}\right)$

To prove the limit in the statement of the theorem, we are left to show

$$
\lim _{N \rightarrow \infty} \int_{\mathcal{C}^{+}} \frac{x^{z}}{\prod_{i=1}^{N} \prod_{j=0}^{\theta-1}\left(1-q^{-z} q^{\lambda_{N-i+1}(N)+\theta(i-1)+j}\right)} \mathrm{d} z=\int_{\mathcal{C}^{+}} x^{z} \prod_{i=1}^{\infty} \frac{\left(q^{-z+\nu_{i}} t^{i} ; q\right)_{\infty}}{\left(q^{-z+\nu_{i}} t^{i-1} ; q\right)_{\infty}} \mathrm{d} z .
$$

We already proved the pointwise convergence; to make use of the dominated convergence theorem, we simply need estimates on the contribution of the tails of $\mathcal{C}^{+}$that are uniform in $N$ and uniform for $x$ belonging to compact subsets of $\mathbb{C} \backslash\{0\}$. Parametrize the tails of $\mathcal{C}^{+}$as $z=r+\frac{\pi \sqrt{-1}}{\ln q}$ or $z=r-\frac{\pi \sqrt{-1}}{\ln q}$; for $r$ ranging from some large $R>1$ to $+\infty$, we want to show that the contribution of each of these lines is small. We have

$$
\begin{aligned}
& \left|\frac{x^{z}}{\prod_{i=1}^{N} \prod_{j=0}^{\theta-1}\left(1-q^{-z} q^{\lambda_{N-i+1}(N)+\theta(i-1)+j}\right)}\right|=\frac{\left|x^{r \pm \frac{\pi \sqrt{-1}}{\ln q}}\right|}{\prod_{i=1}^{N} \prod_{j=0}^{\theta-1}\left(1+q^{-r} q^{\lambda_{N-i+1}(N)+\theta(i-1)+j}\right)} \\
& \leq C_{1} \times \frac{|x|^{r}}{\prod_{i=1}^{k} \prod_{j=0}^{\theta-1}\left(1+q^{-r} q^{\lambda_{N-i+1}(N)+\theta(i-1)+j}\right)} \\
& \leq C_{2} \times \frac{|x|^{r}}{\prod_{i=1}^{k} \prod_{j=0}^{\theta-1}\left(1+q^{-r}\right)} \leq C_{2} \times \frac{|x|^{r}}{\left(q^{-r}\right)^{\theta k}}=C_{2} \times\left(|x| t^{k}\right)^{r},
\end{aligned}
$$

for all $1 \leq k \leq N$, where the constant in the second line is uniform for $x$ over compact subsets of $\mathbb{C} \backslash\{0\}$, and the constant in the third line depends on $k$, but not on $N$ (note that to go 
from the second to the third line, we needed to use that $\lim _{N \rightarrow \infty} \lambda_{N-i+1}(N)$ exists for $i=1, \ldots, k$ and so the sequence $\left\{\lambda_{N-i+1}(N)\right\}_{N>1}$ is uniformly bounded for $\left.i=1, \ldots, k\right)$. Now for any compact set $K \subset \mathbb{C} \backslash\{0\}$ and any $M>0$, there exists $k \in \mathbb{N}$ such that $\sup _{x \in K}|x| \cdot t^{k} \leq e^{-M}$. Since $\int_{R}^{\infty} e^{-M r} \mathrm{~d} r=e^{-M R} / M \stackrel{R \rightarrow \infty}{\longrightarrow} 0$, we have shown that the contribution of the tails of $\mathcal{C}^{+}$is uniformly small over $x$ in compact subsets of $\mathbb{C} \backslash\{0\}$ and for large enough $N$.

We have proved so far that the limit in the theorem holds uniformly for $x$ in compact subsets of $\mathcal{U}$. Since the set $\left\{q^{-1}, q^{-2}, \ldots\right\}$ is discrete and has no accumulation points, Cauchy's integral formula allows us to deduce the uniform convergence in compact subsets of $\mathbb{C} \backslash\{0\}$ as soon as we show that $\Phi^{\nu}(x ; q, t)$ admits an analytic continuation to $\mathbb{C} \backslash\{0\}$.

By virtue of Riemann's theorem of removable singularities, it will suffice to show that $\Phi^{\nu}(x ; q, t)$ is uniformly bounded in an open neighborhood of each pole $q^{-k}$. Let $R>0, R \notin\left\{q^{n}: n \in \mathbb{Z}\right\}$, be arbitrary. From what we have shown so far, it is clear that

$$
\begin{aligned}
\sup _{\substack{\frac{1}{R} \leq|x| \leq R \\
x \in \mathcal{U}}}\left|\Phi^{\nu}(x ; q, t)\right| & \leq \sup _{N \geq 1} \sup _{\frac{1}{R} \leq|x| \leq R}\left|\frac{P_{\lambda(N)}\left(x, t^{-1}, \ldots, t^{1-N}\right)}{P_{\lambda(N)}\left(1, t^{-1}, \ldots, t^{1-N}\right)}\right| \\
& \leq \sup _{N \geq 1} \sup _{\frac{1}{R} \leq|x| \leq R}\left|\frac{P_{\lambda(N)}\left(x, t^{-1}, \ldots, t^{1-N}\right)}{P_{\lambda(N)}\left(1, t^{-1}, \ldots, t^{1-N}\right)}\right| .
\end{aligned}
$$

Thanks to the branching rule for Macdonald polynomials, Theorem 2.5, the fact that all the branching coefficients $\psi_{\mu / \nu}(q, t)$ are nonnegative when $q, t \in(0,1)$, and the fact that each $P_{\lambda(N)}\left(x, t^{-1}, \ldots, t^{1-N}\right)$ is a Laurent polynomial in $x$, we have

$$
\begin{aligned}
\sup _{\frac{1}{R} \leq|x| \leq R}\left|\frac{P_{\lambda(N)}\left(x, t^{-1}, \ldots, t^{1-N}\right)}{P_{\lambda(N)}\left(1, t^{-1}, \ldots, t^{1-N}\right)}\right| & \leq \sup _{\frac{1}{R} \leq|x| \leq R} \frac{P_{\lambda(N)}\left(|x|, t^{-1}, \ldots, t^{1-N}\right)}{P_{\lambda(N)}\left(1, t^{-1}, \ldots, t^{1-N}\right)} \\
& \leq \frac{P_{\lambda(N)}\left(R, t^{-1}, \ldots, t^{1-N}\right)}{P_{\lambda(N)}\left(1, t^{-1}, \ldots, t^{1-N}\right)}+\frac{P_{\lambda(N)}\left(R^{-1}, t^{-1}, \ldots, t^{1-N}\right)}{P_{\lambda(N)}\left(1, t^{-1}, \ldots, t^{1-N}\right)} .
\end{aligned}
$$

From the pointwise limits

$$
\lim _{N \rightarrow \infty} \frac{P_{\lambda(N)}\left(R^{ \pm 1}, t^{-1}, \ldots, t^{1-N}\right)}{P_{\lambda(N)}\left(1, t^{-1}, \ldots, t^{1-N}\right)}=\Phi^{\nu}\left(R^{ \pm 1} ; q, t\right),
$$

we deduce that the sequences $\left\{P_{\lambda(N)}\left(R^{ \pm 1}, t^{-1}, \ldots, t^{1-N}\right) / P_{\lambda(N)}\left(1, t^{-1}, \ldots, t^{1-N}\right)\right\}_{N \geq 1}$ are uniformly bounded. As a result of the estimates above,

$$
\sup _{\substack{\frac{1}{R} \leq|x| \leq R \\ x \in \mathcal{U}}}\left|\Phi^{\nu}(x ; q, t)\right|<\infty .
$$

Thus $\Phi^{\nu}(x ; q, t)$ admits an analytic continuation to all the poles in $\left\{z \in \mathbb{C}: \frac{1}{R} \leq|z| \leq R\right\} \cap$ $\left\{q^{-1}, q^{-2}, \ldots\right\}$. Since $R>0$ was arbitrary (outside of a lattice), we conclude that $\Phi^{\nu}(x ; q, t)$ admits an analytic continuation to $\mathbb{C} \backslash\{0\}$.

If $\nu_{1} \geq 0$, then $\lim _{N \rightarrow \infty} \lambda_{N}(N)=\nu_{1}$ shows that $\lambda_{N}(N) \geq 0$ for all $N>N_{0}$ and $N_{0} \in \mathbb{N}$ large enough. For all $N>N_{0}$, the functions $P_{\lambda(N)}\left(x, t^{-1}, \ldots, t^{1-N}\right) / P_{\lambda(N)}\left(1, t^{-1}, \ldots, t^{1-N}\right)$ are polynomials in $x$ and therefore holomorphic on $\mathbb{C}$. Similar considerations as above allow us to analytically continue $\Phi^{\nu}(x ; q, t)$ to $\mathbb{C}$ in this case, and also show that the limit (5.1) holds uniformly for $x$ belonging to compact subsets of $\mathbb{C}$.

We end this subsection with a Lemma that will be used in Section 7. 
Lemma 5.4. If $\nu, \widetilde{\nu} \in \mathcal{N}$ are such that

$$
\Phi^{\nu}(x ; q, t)=\Phi^{\widetilde{\nu}}(x ; q, t) \quad \forall x \in \mathbb{T},
$$

then $\nu=\widetilde{\nu}$

Proof. In the integral representation of $\Phi^{\nu}(x ; q, t)$, the set of poles of the integrand is

$$
\bigcup_{r=1}^{\infty} \bigcup_{s=0}^{\theta-1}\left\{\nu_{r}+\theta(r-1)+s\right\}
$$

and they are all enclosed by contour $\mathcal{C}^{+}$. We can therefore expand

$$
\frac{\ln q}{2 \pi \sqrt{-1}} \int_{\mathcal{C}^{+}} x^{z} \prod_{i=1}^{\infty} \frac{\left(q^{-z+\nu_{i}} t^{i} ; q\right)_{\infty}}{\left(q^{-z+\nu_{i}} t^{i-1} ; q\right)_{\infty}} \mathrm{d} z
$$

at least formally, as the sum of residues

$$
\sum_{r=1}^{\infty} \sum_{s=0}^{\theta-1}\left\{x^{\nu_{r}+\theta(r-1)+s} \prod_{\substack{i \geq 1 \\ i \neq r}} \frac{\left(q^{-\nu_{r}+\nu_{i}-s} t^{i-r+1} ; q\right)_{\infty}}{\left(q^{-\nu_{r}+\nu_{i}-s} t^{i-r} ; q\right)_{\infty}} \times \prod_{\substack{\theta>j \geq 0 \\ j \neq s}} \frac{1}{\left(1-q^{j-s}\right)}\right\} .
$$

The series above converges absolutely for all $x \in \mathbb{C} \backslash\{0\}$, and uniformly for $x$ in compact subsets of $\mathbb{C} \backslash\{0\}$; in fact, we can argue as in the special case $\theta=1$ of [24, Section 6.2]. We have the bounds

$$
\begin{aligned}
& \prod_{\substack{\theta>j \geq 0 \\
j \neq s}}\left|\frac{1}{\left(1-q^{j-s}\right)}\right| \leq \frac{1}{(1-q) \cdots\left(1-q^{\theta}\right)\left(q^{-1}-1\right) \cdots\left(q^{-\theta}-1\right)} \\
& \prod_{i>r}\left|\frac{\left(q^{-\nu_{r}+\nu_{i}-s} t^{i-r+1} ; q\right)_{\infty}}{\left(q^{-\nu_{r}+\nu_{i}-s} t^{i-r} ; q\right)_{\infty}}\right|=\prod_{i>r}^{\theta-1}\left|\frac{1}{j=0}\right| \\
& \quad=\prod_{i>r} \prod_{j=0}^{\theta-1} \frac{1}{\left(1-q^{-\nu_{r}+\nu_{i}-s+\theta(i-r)+j}\right)} \mid \\
& \prod_{i<r}\left|\frac{\left(q^{-\nu_{r}+\nu_{i}-s} t^{i-r+1} ; q\right)_{\infty}}{\left(q^{-\nu_{r}+\nu_{i}-s} t^{i-r} ; q\right)_{\infty}}\right|=\prod_{i<r} \prod_{j=0}^{\theta-\theta} \frac{q^{\nu_{r}+\theta(r-1)+s-\nu_{i}-\theta(i-1)-j}}{1-q^{\nu_{r}-\nu_{i}+\theta(r-i)-j+s}} \\
& \left.\quad \leq \frac{1}{(q ; q)_{\infty}} \prod_{i<r}^{\theta-1} \frac{1}{\left(1-\prod_{j=0}\right.} q^{\nu_{r}+\theta(r-1)+s-\nu_{i}-\theta(i-1)-j}\right) \\
& \quad \leq \frac{1}{(q ; q)_{\infty}} \prod_{i=1}^{m} \prod_{j=0}^{\theta-1} q^{\nu_{r}+\theta(r-1)+s-\nu_{i}-\theta(i-1)-j}, \quad \text { for any } \quad 1 \leq m<r \\
& \quad=q^{m \theta\left(\nu_{r}+\theta(r-1)+s\right)} \times \frac{q^{-\theta\left(\nu_{1}+\cdots+\nu_{m}\right)} q^{-\left(\begin{array}{c}
\theta m \\
2
\end{array}\right)}}{(q ; q)_{\infty}} .
\end{aligned}
$$

Choose an arbitrary $m \in \mathbb{N}$ and fix it. For $r>m$, the general term in brackets at (5.5) has modulus upper bounded by

$$
\left(|x| q^{m \theta}\right)^{\nu_{r}+\theta(r-1)+s} \times c(m, \theta ; q),
$$


where $c(m, \theta ; q)>0$ is a constant depending on $m, \theta$, as well as $\nu_{1}, \ldots, \nu_{m}$, but not on $r$. It follows that the sum (5.5) converges absolutely for any $x \in \mathbb{C} \backslash\{0\}$ with $|x|<q^{-m \theta}$. Since $m \in \mathbb{N}$ was arbitrary, it follows that (5.5) converges absolutely for any $x \in \mathbb{C} \backslash\{0\}$. The uniform convergence also follows from the bound above. In particular, (5.5) is the Fourier expansion of $\frac{(x q ; q)_{\infty}}{(q ; q)_{\infty}} \Phi^{\nu}(x ; q, t)$. A similar Fourier expansion can be given for $\frac{(x q ; q)_{\infty}}{(q ; q)_{\infty}} \Phi^{\widetilde{\nu}}(x ; q, t)$. After multiplying equality $(5.4)$ by $(x q ; q)_{\infty} /(q ; q)_{\infty}$, we have

$$
\frac{\ln q}{2 \pi \sqrt{-1}} \int_{\mathcal{C}^{+}} x^{z} \prod_{i=1}^{\infty} \frac{\left(q^{-z+\nu_{i}} t^{i} ; q\right)_{\infty}}{\left(q^{-z+\nu_{i}} t^{i-1} ; q\right)_{\infty}} \mathrm{d} z=\frac{\ln q}{2 \pi \sqrt{-1}} \int_{\mathcal{C}^{+}} x^{z} \prod_{i=1}^{\infty} \frac{\left(q^{-z+\widetilde{\nu}_{i}} t^{i} ; q\right)_{\infty}}{\left(q^{-z+\widetilde{\nu}_{i}} t^{i-1} ; q\right)_{\infty}} \mathrm{d} z .
$$

We can expand both sides of (5.6) as above, to get an equality of the form $\sum_{k \in \mathbb{Z}} c_{k}(\nu) x^{k}=$ $\sum_{k \in \mathbb{Z}} c_{k}(\widetilde{\nu}) x^{k}$. More precisely, (5.5) gives that the set $\left\{k \in \mathbb{Z}: c_{k}(\nu) \neq 0\right\}$ of indices which appear in the expansion of the left-hand side of (5.6) is $\left\{\nu_{r}+\theta(r-1)+s: 1 \leq r, 0 \leq s \leq \theta-1\right\}$. Similarly, $\left\{k \in \mathbb{Z}: c_{k}(\widetilde{\nu}) \neq 0\right\}=\left\{\widetilde{\nu}_{r}+\theta(r-1)+s: 1 \leq r, 0 \leq s \leq \theta-1\right\}$. The equality of these sets, and the inequalities $\nu_{1} \leq \nu_{2} \leq \cdots, \widetilde{\nu}_{1} \leq \widetilde{\nu}_{2} \leq \cdots$, imply that $\nu_{r}=\widetilde{\nu}_{r}$ for all $r \geq 1$, i.e., $\nu=\widetilde{\nu}$.

\subsection{Asymptotics of Macdonald characters of a fixed number $m$ of variables}

The following theorem is the main result for asymptotics of Macdonald characters of any given rank $m \in \mathbb{N}$ as $N$ tends to infinity.

Theorem 5.5. Let $\theta \in \mathbb{N}, t=q^{\theta}$, and $\{\lambda(N)\}_{N \geq 1}, \lambda(N) \in \mathbb{G}_{N}$, be a sequence of signatures that stabilizes to $\nu \in \mathcal{N}$. Also let $m \in \mathbb{N}$ be arbitrary. Then

$$
\lim _{N \rightarrow \infty} \frac{P_{\lambda(N)}\left(x_{1}, \ldots, x_{m}, t^{-m}, \ldots, t^{1-N}\right)}{P_{\lambda(N)}\left(1, t^{-1}, t^{-2}, \ldots, t^{1-N}\right)}=\Phi^{\nu}\left(x_{1}, \ldots, x_{m} ; q, t\right),
$$

where

$$
\begin{aligned}
& \Phi^{\nu}\left(x_{1}, \ldots, x_{m} ; q, t\right) \stackrel{\text { def }}{=} \frac{q^{-2 \theta^{2}\left(\begin{array}{c}
m \\
3
\end{array}\right)-\left(\begin{array}{c}
\theta+1 \\
2
\end{array}\right)\left(\begin{array}{c}
m \\
2
\end{array}\right)}}{\prod_{i=1}^{m}\left(x_{i} q t^{m-1} ; q\right)_{\infty}} \frac{1}{\prod_{\substack{1 \leq i<j \leq m \\
0 \leq k<\theta}}\left(q^{k} x_{j}-x_{i}\right)} \\
& \times \widetilde{D}_{q, \theta}^{(m)}\left\{\prod_{i=1}^{m} \Phi^{\nu}\left(x_{i} ; q, t\right)\left(x_{i} q ; q\right)_{\infty}\right\}, \\
& \widetilde{D}_{q, \theta}^{(m)} \stackrel{\text { def }}{=} \sum_{\tau \in M_{\theta}^{(m)}} C_{\tau}^{\left(q, q^{\theta}\right)}\left(x_{1}, \ldots, x_{m}\right) \prod_{i=1}^{m} T_{q, x_{i}}^{(i-1) \theta+\tau_{i}^{+}-\tau_{i}^{-}} .
\end{aligned}
$$

Above we used the notation of Section 4: $M_{\theta}^{(m)}$ is the set of all strictly upper triangular matrices with entries in $\{0,1, \ldots, \theta\}$, and for $\tau \in M_{\theta}^{(m)}$, we let $\tau_{i}^{+} \stackrel{\text { def }}{=} \sum_{j>i} \tau_{i, j}, \tau_{i}^{-} \stackrel{\text { def }}{=} \sum_{k<i} \tau_{k, i}$. Also for $\tau \in M_{\theta}^{(m)}$, let $C_{\tau}^{\left(q, q^{\theta}\right)}\left(x_{1}, \ldots, x_{m}\right)$ be the rational functions defined in (4.3). The function $\Phi^{\nu}\left(x_{1}, \ldots, x_{m} ; q, t\right)$ is defined by the formula above in the domain

$$
\mathcal{U}_{m} \stackrel{\text { def }}{=} \bigcap_{k \in \mathbb{Z}} \bigcap_{i<j}\left\{x_{i} \neq q^{k} x_{j}\right\} \cap \bigcap_{k \geq 1} \bigcap_{i=1}^{m}\left\{x_{i} \neq q^{-k} t^{1-m}\right\} \cap \bigcap_{i=1}^{m}\left\{x_{i} \neq 0\right\},
$$

and admits an analytic continuation to the domain $\bigcap_{i=1}^{m}\left\{x_{i} \neq 0\right\}=(\mathbb{C} \backslash\{0\})^{m}$. Moreover if $\nu_{1} \geq 0$, the function $\Phi^{\nu}\left(x_{1}, \ldots, x_{m} ; q, t\right)$ can be analytically continued to $\mathbb{C}^{m}$. 
The convergence (5.7) is uniform on compact subsets of $(\mathbb{C} \backslash\{0\})^{m}$ and if $\nu_{1} \geq 0$, then it is uniform on compact subsets of $\mathbb{C}^{m}$.

Remark 5.6. For $\theta=1$, our theorem has a different form than [24, Theorem 6.5]. It is not immediately clear that the two answers are the same.

Remark 5.7. From their definition, it is clear that the rational functions $\left\{C_{\tau}^{\left(q, q^{\theta}\right)}\left(x_{1}, \ldots, x_{m}\right)\right.$ : $\left.\tau \in M_{\theta}^{(m)}\right\}$ are holomorphic in a domain of the form $\bigcap_{-N_{1} \leq k \leq N_{1}} \bigcap_{i<j}\left\{x_{i} \neq q^{k} x_{j}\right\}$, for large enough $N_{1} \in \mathbb{N}$. In particular, all functions $\left\{C_{\tau}^{\left(q, q^{\theta}\right)}\left(x_{1}, \ldots, x_{m}\right): \tau \in M_{\theta}^{(m)}\right\}$ are holomorphic on $\mathcal{U}_{m}$.

Proof. This result is a consequence of Theorem 5.2 and the multiplicative formula for Macdonald polynomials, Theorem 4.1. Let us give more details. As before we prove first the uniform limit on compact subsets of $\mathcal{U}_{m}$. Begin by applying Theorem 4.1 for the signature $\lambda(N) \in \mathbb{G T}_{N}$ and $t^{N-1} x_{i}$ instead of $x_{i}$, for $i=1, \ldots, m$. Since $P_{\lambda(N)}$ is a homogeneous Laurent polynomial of degree $|\lambda(N)|$, the resulting left-hand side is

$$
\frac{P_{\lambda(N)}\left(x_{1}, \ldots, x_{m}, t^{-m}, \ldots, t^{1-N}\right)}{P_{\lambda(N)}\left(1, t^{-1}, t^{-2}, \ldots, t^{1-N}\right)} .
$$

As for the right side, the factor

$$
\prod_{\substack{1 \leq i<j \leq m \\
0 \leq k<\theta}}\left(t^{N-1} x_{i}-t^{N-1} x_{j} q^{k}\right)^{-1} \times(q-1)^{-\theta\left(\begin{array}{c}
m \\
2
\end{array}\right)} \times \prod_{i=1}^{m} \frac{[\theta(N-i+1)-1]_{q} !}{[\theta N-1]_{q} !}
$$

equals

$$
(-1)^{\theta\left(\begin{array}{c}
m \\
2
\end{array}\right)} \frac{q^{-\theta^{2}(N-1)\left(\begin{array}{c}
m \\
2
\end{array}\right)}}{\prod_{\substack{1 \leq i<j \leq m \\
0 \leq k<\theta}}\left(x_{i}-q^{k} x_{j}\right)} \prod_{i=1}^{m} \frac{1}{\left(t^{N-i+1} ; q\right)_{\theta(i-1)}}
$$

We can also obtain easily the polynomial equality

$$
\prod_{j=1}^{k}\left(t^{N-1} x-q^{j-\theta}\right)=(-1)^{k} q^{\left(\begin{array}{c}
k+1 \\
2
\end{array}\right)-\theta k} \prod_{i=1}^{k}\left(1-x q^{\theta N-i}\right), \quad \text { for any } \quad k \in \mathbb{N} \text {. }
$$

Therefore for any $k \in \mathbb{N}$, we have

$$
\prod_{i=1}^{m} \prod_{j=1}^{k-1}\left(x_{i} t^{N-1}-q^{j-\theta}\right)=(-1)^{m(k-1)} q^{m\left(\left(\begin{array}{c}
k \\
2
\end{array}\right)-\theta(k-1)\right)} \prod_{i=1}^{m}\left(x_{i} q^{\theta N-k+1} ; q\right)_{k-1} .
$$

Observe also that the rational functions are invariant under the simultaneous transformations $x_{i} \mapsto t^{N-1} x_{i} \forall i=1,2, \ldots, m$, that is

$$
C_{\tau}^{\left(q, q^{\theta}\right)}\left(t^{N-1} x_{1}, \ldots, t^{N-1} x_{m}\right)=C_{\tau}^{\left(q, q^{\theta}\right)}\left(x_{1}, \ldots, x_{m}\right) .
$$

By combining (5.9), (5.10), (5.11) for $k=\theta(N-m+1), \theta N$, and (5.12), we obtain

$$
\frac{P_{\lambda(N)}\left(x_{1}, \ldots, x_{m}, t^{-m}, \ldots, t^{1-N}\right)}{P_{\lambda(N)}\left(1, t^{-1}, t^{-2}, \ldots, t^{1-N}\right)}=(-1)^{\theta\left(\begin{array}{c}
m \\
2
\end{array}\right)} q^{-2 \theta^{2}\left(\begin{array}{c}
m \\
3
\end{array}\right)-\left(\begin{array}{c}
\theta+1 \\
2
\end{array}\right)\left(\begin{array}{c}
m \\
2
\end{array}\right)} \times \frac{1}{\prod_{i=1}^{m}\left(t^{N-i+1} ; q\right)_{\theta(i-1)}}
$$




$$
\begin{aligned}
& \times \frac{1}{\prod_{\substack{1 \leq i<j \leq m \\
0 \leq k<\theta}}\left(x_{i}-q^{k} x_{j}\right)} \frac{1}{\prod_{i=1}^{m}\left(x_{i} t^{m-1} q ; q\right)_{\theta(N-m+1)-1}} \\
& \times \widetilde{D}_{q, \theta}^{(m)}\left\{\prod_{i=1}^{m} \frac{P_{\lambda(N)}\left(x_{i}, t^{-1}, \ldots, t^{1-N}\right)}{P_{\lambda(N)}\left(1, t^{-1}, \ldots, t^{1-N}\right)}\left(x_{i} q ; q\right)_{\theta N-1}\right\} .
\end{aligned}
$$

The following limits hold uniformly for $\left(x_{1}, \ldots, x_{m}\right)$ belonging to compact subsets of $\mathcal{U}_{m}$

$$
\lim _{N \rightarrow \infty} \prod_{i=1}^{m}\left(t^{N-1+1} ; q\right)_{\theta(i-1)}=1, \quad \lim _{N \rightarrow \infty} \frac{1}{\prod_{i=1}^{m}\left(x_{i} t^{m-1} q ; q\right)_{\theta(N-m+1)-1}}=\frac{1}{\prod_{i=1}^{m}\left(x_{i} t^{m-1} q ; q\right)_{\infty}} .
$$

We have moreover the following limit holds uniformly for $\left(x_{1}, \ldots, x_{m}\right)$ belonging to compact subsets of $(\mathbb{C} \backslash\{0\})^{m}$, because of Theorem 5.2,

$$
\lim _{N \rightarrow \infty} \prod_{i=1}^{m} \frac{P_{\lambda(N)}\left(x_{i}, t^{-1}, \ldots, t^{1-N}\right)}{P_{\lambda(N)}\left(1, t^{-1}, \ldots, t^{1-N}\right)}\left(x_{i} q ; q\right)_{\theta N-1}=\prod_{i=1}^{m} \Phi^{\nu}\left(x_{i} ; q, t\right)\left(x_{i} q ; q\right)_{\infty} .
$$

It is not difficult to observe that if $U \subset \mathbb{C}^{m}$ is a domain preserved by the map of multiplication by $q$, and $\left\{f_{n}\right\}_{n \geq 1}, f$ are sequences of holomorphic functions on $U$ for which $\lim _{n \rightarrow \infty} f_{n}(x)=f(x)$ uniformly on compact subsets of $U$, then

$$
\lim _{n \rightarrow \infty} T_{q, x}^{s} f_{n}(x)=\lim _{n \rightarrow \infty} f_{n}\left(q^{s} x\right)=f\left(q^{s} x\right)=T_{q, x}^{s} f(x)
$$

uniformly for $x$ belonging to compact subsets of $U$. As an implication, the order of the limit as $N \rightarrow \infty$ and the $q$-difference operator $\widetilde{D}_{q, \theta}^{(m)}$ can be interchanged. All the considerations above immediately imply the desired uniform limit for $\left(x_{1}, \ldots, x_{m}\right)$ belonging to compact subsets of $\mathcal{U}_{m}$.

As in the proof of Theorem 5.2, the limit in the statement will hold also uniformly for compact subsets of $(\mathbb{C} \backslash\{0\})^{m}$ if we show that $\Phi^{\nu}\left(x_{1}, \ldots, x_{m} ; q, t\right)$ admits an analytic continuation to this larger domain. The extension of Riemann's theorem for removable singularities for several complex variables, [45, Theorem 8], shows that $\Phi^{\nu}\left(x_{1}, \ldots, x_{m} ; q, t\right)$ admits an analytic continuation to all $(\{z \in \mathbb{C} \backslash\{0\}:|z| \leq R\})^{m}$ if we showed only that $\Phi^{\nu}\left(x_{1}, \ldots, x_{m} ; q, t\right)$ is bounded on $(\{z \in$ $\mathbb{C} \backslash\{0\}:|z| \leq R\})^{m} \cap \mathcal{U}_{m}$. The latter can be proved by repeating the argument in the proof of Theorem 5.2 above.

Finally, if $\nu_{1} \geq 0$ then $\lim _{N \rightarrow \infty} \lambda_{N}(N)=\nu_{1}$ implies $\lambda(N)_{N} \geq 0$ for large enough $N$. It follows that $P_{\lambda(N)}\left(x_{1}, \ldots, x_{m}, t^{-m}, \ldots, t^{1-N}\right) / P_{\lambda(N)}\left(1, t^{-1}, \ldots, t^{1-N}\right)$ is a polynomial in $x_{1}, \ldots, x_{m}$ for large enough $N$, and therefore holomorphic on $\mathbb{C}^{m}$. The same argument as in the proof of Theorem 5.2 again shows $\Phi^{\nu}\left(x_{1}, \ldots, x_{m} ; q, t\right)$ admits an analytic continuation to $\mathbb{C}^{m}$ and the limit holds uniformly for compact subsets of $\mathbb{C}^{m}$.

\section{Preliminaries on the $(q, t)$-Gelfand-Tsetlin graph}

In this section, assume $q, t \in(0,1)$. We use the notation $\mathbb{P}-\lim _{k \rightarrow \infty} M_{k}=M$ to indicate that a sequence of probability measures $\left\{M_{k}\right\}_{k \geq 1}$ converges weakly to $M$.

\subsection{The $(q, t)$-Gelfand-Tsetlin graph}

The $(q, t)$-Gelfand-Tsetlin graph is an undirected, $\mathbb{Z}_{\geq 0}$-graded graph with countable vertices, together with a sequence of cotransition probabilities between the levels of the graph (considered as discrete spaces). 
Begin by defining the set of vertices of the graph as the set of all signatures

$$
\mathbb{G} \mathbb{T} \stackrel{\text { def }}{=} \bigsqcup_{N \geq 0} \mathbb{G} \mathbb{T}_{N}
$$

where, for convenience, we have also included the singleton $\mathbb{G T}_{0} \stackrel{\text { def }}{=}\{\varnothing\}$.

The edges are determined by the interlacing constraints: edges only join vertices associated to signatures whose lengths differ by 1 and $\mu \in \mathbb{G T}_{N}$ is joined to $\lambda \in \mathbb{G}_{N+1}$ if and only if $\mu \prec \lambda$, i.e., $\lambda_{N+1} \leq \mu_{N} \leq \cdots \leq \lambda_{2} \leq \mu_{1} \leq \lambda_{1}$. We also assume $\varnothing \prec(k), \forall(k) \in \mathbb{G}_{1}$. We call the graph with vertices and edges just described the Gelfand-Tsetlin graph ${ }^{2}$. Next we introduce a $(q, t)$-deformation of the GT graph by considering certain cotransition probabilities.

Definition 6.1. Consider the numbers $\Lambda_{N}^{N+1}(\lambda, \mu), \mu \prec \lambda$, defined by the expression

$$
\Lambda_{N}^{N+1}(\lambda, \mu)=t^{|\mu|} \psi_{\lambda / \mu}(q, t) \frac{P_{\mu}\left(1, t, \ldots, t^{N-1}\right)}{P_{\lambda}\left(1, t, \ldots, t^{N}\right)}=\psi_{\lambda / \mu}(q, t) \frac{P_{\mu}\left(t, t^{2}, \ldots, t^{N}\right)}{P_{\lambda}\left(1, t, \ldots, t^{N}\right)},
$$

for all $N \in \mathbb{Z}_{\geq 0}, \lambda \in \mathbb{G T}_{N+1}, \mu \in \mathbb{G T}_{N}, \mu \prec \lambda$, and where the branching coefficients $\psi_{\lambda / \mu}(q, t)$ are defined in Theorem 2.5. For convenience, also let $\Lambda_{N}^{N+1}(\lambda, \mu)=0$ if $\mu \nprec \lambda$, i.e., if $\mu$ is not adjacent to $\lambda$.

The $(q, t)$-Gelfand-Tsetlin graph $h^{3}$ is the sequence $\left\{\mathbb{G T}_{N}, \Lambda_{N}^{N+1}: N=0,1,2, \ldots\right\}$ of data consisting of the GT graph and the $\mathbb{G T}_{N+1} \times \mathbb{G T}_{N}$ matrices $\left[\Lambda_{N}^{N+1}(\lambda, \mu)\right], N \geq 0$, defined above.

In general, the numbers $\Lambda_{N}^{N+1}(\lambda, \mu)$ depend on the values $q, t$, but for simplicity we omit that dependence from the notation. By virtue of the evaluation identity, Theorem 2.3, and the assumption $q, t \in(0,1)$, we have

$$
\Lambda_{N}^{N+1}(\lambda, \mu) \geq 0, \quad \forall \lambda \in \mathbb{G}_{N+1}, \quad \mu \in \mathbb{G}_{N}
$$

Moreover, the branching rule for Macdonald polynomials, Theorem 2.5, shows

$$
P_{\lambda}\left(1, t, t^{2}, \ldots, t^{N}\right)=\sum_{\mu: \mu \prec \lambda} \psi_{\lambda / \mu}(q, t) P_{\mu}\left(t, t^{2}, \ldots, t^{N}\right)
$$

and then

$$
1=\sum_{\mu: \mu \prec \lambda} \Lambda_{N}^{N+1}(\lambda, \mu)
$$

Equations (6.2) and (6.3) show that $\left[\Lambda_{N}^{N+1}(\lambda, \mu)\right]$ is a stochastic matrix of format $\mathbb{G T}_{N+1} \times \mathbb{G T}_{N}$, for each $N \geq 0$. Thus $\Lambda_{N}^{N+1}$ determines a Markov kernel $\mathbb{G T}_{N+1} \rightarrow \mathbb{G}_{N}$. For this reason, we call $\Lambda_{N}^{N+1}(\lambda, \mu)$ the cotransition probabilities. Let us also define the more general Markov kernels $\Lambda_{N}^{M}: \mathbb{G}_{M} \rightarrow \mathbb{G}_{N}, M>N$, by

$$
\Lambda_{N}^{M} \stackrel{\text { def }}{=} \Lambda_{M-1}^{M} \Lambda_{M-2}^{M-1} \cdots \Lambda_{N}^{N+1}
$$

or more explicitly

$$
\Lambda_{N}^{M}(\lambda, \mu) \stackrel{\text { def }}{=} \sum_{\lambda \succ \lambda^{(M-1)} \succ \cdots \succ \lambda^{(N+1)} \succ \mu} \Lambda_{M-1}^{M}\left(\lambda, \lambda^{(M-1)}\right) \cdots \Lambda_{N}^{N+1}\left(\lambda^{(N+1)}, \mu\right) .
$$

\footnotetext{
${ }^{2} \mathrm{GT}$ graph, for short.

${ }^{3}(q, t)$-GT graph, for short.
} 
By duality, the kernel $\Lambda_{N}^{M}$ also determines a map $\mathcal{M}_{\text {prob }}\left(\mathbb{G} \mathbb{T}_{M}\right) \rightarrow \mathcal{M}_{\text {prob }}\left(\mathbb{G T}_{N}\right)$ between the spaces of probability measures on $\mathbb{G T}_{M}$ and $\mathbb{G T}_{N}$, that we denote by the same symbol $\Lambda_{N}^{M}$. For example, if $\lambda \in \mathbb{G T}_{M}$ and $\delta_{\lambda}$ is the delta mass at $\lambda$, then $\Lambda_{N}^{M} \delta_{\lambda}$ is the probability measure on $\mathbb{G T}_{N}$ given by

$$
\Lambda_{N}^{M} \delta_{\lambda}(\mu)=\Lambda_{N}^{M}(\lambda, \mu)
$$

Definition 6.2. A sequence $\left\{M_{N}\right\}_{N \geq 0}$, such that each $M_{N}$ is a probability measure on $\mathbb{G T}_{N}$, is called a $(q, t)$-coherent sequence if the following relations are satisfied

$$
M_{N}(\mu)=\sum_{\lambda \in \mathbb{G}_{N+1}} M_{N+1}(\lambda) \Lambda_{N}^{N+1}(\lambda, \mu), \quad \forall N \geq 0, \quad \forall \mu \in \mathbb{G}_{N}
$$

Similarly, a finite sequence $\left\{M_{N}\right\}_{N=0,1, \ldots, k}$ is said to be a $(q, t)$-coherent sequence if the relations (6.5) hold for $N=0,1, \ldots, k-1$ and all $\mu \in \mathbb{G T}_{N}$.

It is clear that for any probability measure $M_{N}$ on $\mathbb{G T}_{N}$, there exist probability measures $M_{0}, M_{1}, \ldots, M_{N-1}$ on $\mathbb{G}_{0}, \mathbb{G}_{1}, \ldots, \mathbb{G}_{N-1}$ such that $\left\{M_{m}\right\}_{m=0,1, \ldots, N}$ is a $(q, t)$-coherent sequence, and moreover $M_{0}, \ldots, M_{N-1}$ are uniquely determined by this condition: in fact, $M_{m}=\Lambda_{m}^{N} M_{N} \forall 0 \leq m \leq N-1$.

The set of (infinite) $(q, t)$-coherent sequences $\left\{M_{N}\right\}_{N \geq 0}$ is a convex set. Theorem 1.3 is, in different terms, a characterization of the extreme points of the set of $(q, t)$-coherent sequences.

\subsection{The path-space $\mathcal{T}$ and $(q, t)$-central measures}

The set of $(q, t)$-coherent sequences defined before is in bijection with a class of probability measures in the path-space of the GT graph $\mathcal{T}$ that we define next.

Definition 6.3. The path-space $\mathcal{T}$ is the set of (infinite) paths in the GT graph that begin at $\varnothing \in \mathbb{G T}_{0}$ :

$$
\mathcal{T} \stackrel{\text { def }}{=}\left\{\tau=\left(\varnothing=\tau^{(0)} \prec \tau^{(1)} \prec \tau^{(2)} \prec \cdots\right): \tau^{(n)} \in \mathbb{G}_{n} \forall n \geq 0\right\} .
$$

For any finite path $\phi=\left(\phi^{(0)} \prec \phi^{(1)} \prec \cdots \prec \phi^{(n)}\right)$, define the cylinder set $S_{\phi}$ (or simply $S(\phi)$ ) by

$$
S_{\phi} \stackrel{\text { def }}{=}\left\{\tau \in \mathcal{T}: \tau^{(i)}=\phi^{(i)} \forall i=0,1, \ldots, n\right\} \subset \mathcal{T} \text {. }
$$

The set $\mathcal{T}$ is equipped with the $\sigma$-algebra generated by the cylinder sets $S_{\phi}$, where $\phi$ varies over all finite paths in the GT graph. We always consider $\mathcal{T}$ as a measurable space.

An interesting class of probability measures on $\mathcal{T}$ consists of the ones that are coherent with the sequence of stochastic matrices $\left\{\Lambda_{N}^{N+1}\right\}_{N \geq 0}$. To clarify what such coherence is, define the natural projection maps

$$
\begin{aligned}
\operatorname{Proj}_{N}: & \mathcal{T} \subset \prod_{n \geq 0} \mathbb{G}_{n} \longrightarrow \mathbb{G T}_{N}, \\
\tau & =\left(\tau^{(0)} \prec \tau^{(1)} \prec \tau^{(2)} \prec \cdots\right) \mapsto \tau^{(N)} .
\end{aligned}
$$

It is a standard exercise to show that the $\sigma$-algebra of $\mathcal{T}$ is the smallest one for which all the maps $\operatorname{Proj}_{N}$ are measurable. Consequently, for any probability measure $M$ on $\mathcal{T}$, we can associate to it the sequence of its pushforwards under the maps $\operatorname{Proj}_{N}$, namely the sequence $\left\{M_{N}\right\}_{N \geq 0}$, $M_{N}=\left(\operatorname{Proj}_{N}\right)_{*} M$. 
Definition 6.4. A probability measure $M$ on $\mathcal{T}$ is said to be a $(q, t)$-central measure if the following relations hold

$$
\begin{aligned}
& M\left(S\left(\phi^{(0)} \prec \phi^{(1)} \cdots \prec \phi^{(N-1)} \prec \phi^{(N)}\right)\right)=\Lambda_{N-1}^{N}\left(\phi^{(N)}, \phi^{(N-1)}\right) \cdots \Lambda_{0}^{1}\left(\phi^{(1)}, \phi^{(0)}\right) M_{N}\left(\phi^{(N)}\right) \\
& \quad=t^{\left|\phi^{(N-1)}\right|+\cdots+\left|\phi^{(0)}\right|} \frac{\psi_{\phi^{(N)} / \phi^{(N-1)}}(q, t) \cdots \psi_{\phi^{(1)} / \phi^{(0)}}(q, t)}{P_{\phi^{(N)}}\left(1, t, \ldots, t^{N-1}\right)} M_{N}\left(\phi^{(N)}\right),
\end{aligned}
$$

for all $N \geq 0$, all finite paths $\phi=\left(\phi^{(0)} \prec \cdots \prec \phi^{(N)}\right)$, and for some probability measures $M_{N}$ on $\mathbb{G}_{N}$. The branching coefficients $\psi_{\mu / \nu}(q, t)$ are explicit in the statement of Theorem 2.5.

One can verify easily that, if the relations above hold, then the measure $M_{N}$ is the pushforward $\left(\operatorname{Proj}_{N}\right)_{*} M$, for all $N \geq 0$. Moreover, $\left\{M_{N}\right\}_{N \geq 0}$ is automatically a $(q, t)$-coherent sequence.

We denote by $M_{\text {prob }}(\mathcal{T})$ the set of $(q, t)$-central (probability) measures. The set of $(q, t)$-central measures is a convex set. The set of extreme points of $M_{\text {prob }}(\mathcal{T})$, equipped with its inherited topology, is called the boundary of the $(q, t)-G T$ graph $^{4}$ and is denoted by $\Omega_{q, t}$.

The following proposition implies that the correspondence between the set of $(q, t)$-central measures and the set of $(q, t)$-coherent sequences is a bijection.

Proposition 6.5. Any probability measure $M$ on $\mathcal{T}$ has an associated sequence $\left\{M_{N}\right\}_{N \geq 0}$ of probability measures on $\left\{\mathbb{G}_{N}\right\}_{N \geq 0}$, as shown above. The map $M \mapsto\left\{M_{N}\right\}_{N \geq 0}$ is a bijection between the set $M_{\text {prob }}(\mathcal{T})$ of $(q, t)$-central measures on $\mathcal{T}$, and the set of $(q, t)$-coherent sequences. The bijection is an isomorphism of convex sets.

Let $\left\{M^{(i)}\right\}_{i>1}, M$ be elements on $M_{\mathrm{prob}}(\mathcal{T})$ and $\left\{M_{m}^{(i)}\right\}_{i>1, m>0},\left\{M_{m}\right\}_{m \geq 0}$ be their corresponding $(q, t)$-coherent sequences. Then the weak limit $\mathbb{P}-\lim _{i \rightarrow \infty} M^{(i)}=M$ holds if and only if the weak limits $\mathbb{P}-\lim _{i \rightarrow \infty} M_{m}^{(i)}=M_{m}$ hold for all $m \in \mathbb{N}$.

Proof. Similar statements are known for other branching graphs, e.g., the case $t=q$ of our proposition is given in [21, Propositions 4.4 and 4.9], and the case $t=q \rightarrow 1$ is in [40, Proposition 10.3]. In the case $t=q$, this proposition is given in [21, Propositions 4.4 and 4.9]. The proof at our level of generality can be easily adapted from the proofs in [21]; details are left to the reader.

\subsection{Macdonald generating functions}

We introduce Macdonald generating functions, which will be very helpful in our study of $(q, t)$ coherent sequences.

Definition 6.6. Let $M_{N}$ be a probability measure on $\mathbb{G T}_{N}$, then its Macdonald generating function is the formal sum

$$
\mathcal{P}_{M_{N}}\left(x_{1}, \ldots, x_{N}\right) \stackrel{\text { def }}{=} \sum_{\lambda \in \mathbb{G}_{N}} M_{N}(\lambda) \frac{P_{\lambda}\left(x_{1}, x_{2} t, \ldots, x_{N} t^{N-1}\right)}{P_{\lambda}\left(1, t, \ldots, t^{N-1}\right)} .
$$

Note that $\mathcal{P}_{M_{m}}\left(x_{1}, \ldots, x_{m}\right)$ depends on the values $q, t$, but we omit such dependence for simplicity.

The sum above is absolutely convergent on the torus $\left(x_{1}, \ldots, x_{m}\right) \in \mathbb{T}^{m}$. In fact, Theorem 2.5 and the fact that all the branching coefficients $\psi_{\mu / \nu}(q, t)$ are nonnegative imply $\left|P_{\lambda}\left(x_{1}, x_{2} t, \ldots, x_{N} t^{N-1}\right)\right| \leq P_{\lambda}\left(\left|x_{1}\right|,\left|x_{2} t\right|, \ldots,\left|x_{N} t^{N-1}\right|\right)=P_{\lambda}\left(1, t, \ldots, t^{N-1}\right)$. Thus not only is

\footnotetext{
${ }^{4}$ We may also call it the minimal boundary of the $(q, t)$-GT graph, to differentiate it from the Martin boundary of the $(q, t)$-GT graph, see Section 7.1 below.
} 
$\mathcal{P}_{M_{N}}\left(x_{1}, \ldots, x_{N}\right)$ well-defined as a function on $\mathbb{T}^{N}$, but also $\sup _{x_{1}, \ldots, x_{N} \in \mathbb{T}}\left|\mathcal{P}_{M_{N}}\left(x_{1}, \ldots, x_{N}\right)\right| \leq 1$.

Therefore $\mathcal{P}_{M_{N}} \in L^{\infty}\left(\mathbb{T}^{m}\right)$.

If $M_{N}$ is supported on the set of positive signatures $\mathbb{G}_{N}^{+}$, then each $P_{\lambda}\left(x_{1}, x_{2} t, \ldots, x_{N} t^{N-1}\right)$ is a polynomial in $x_{1}, \ldots, x_{N}$ and therefore the sum defining $\mathcal{P}_{M_{N}}$ is absolutely convergent on the closed unit disk $\left(x_{1}, \ldots, x_{m}\right) \in \mathbb{D}^{m}$. Moreover $\mathcal{P}_{M_{N}} \in L^{\infty}\left(\mathbb{D}^{m}\right)$ if $M_{N}$ is supported on $\mathbb{G T}_{N}^{+}$.

In general, $\mathcal{P}_{M_{N}} \in L^{\infty}\left(\mathbb{T}^{m}\right) \subset L^{2}\left(\mathbb{T}^{m}\right)$. The Fourier series expansion of $\mathcal{P}_{M_{N}}$ can be obtained by using Corollary 2.6. In fact,

$$
\begin{aligned}
\mathcal{P}_{M_{N}}\left(x_{1}, \ldots, x_{N}\right) & =\sum_{\lambda \in \mathbb{G T}_{N}} M_{N}(\lambda) \frac{P_{\lambda}\left(x_{1}, x_{2} t, \ldots, x_{N} t^{N-1}\right)}{P_{\lambda}\left(1, t, \ldots, t^{N-1}\right)} \\
& =\sum_{\lambda \in \mathbb{G T}_{N}} M_{N}(\lambda) \sum_{\mu \in \mathbb{G T}_{N}} \frac{c_{\lambda, \mu} m_{\mu}\left(x_{1}, \ldots, x_{N} t^{N-1}\right)}{P_{\lambda}\left(1, t, \ldots, t^{N-1}\right)} \\
& =\sum_{\mu \in \mathbb{G T}_{N}} m_{\mu}\left(x_{1}, \ldots, x_{N} t^{N-1}\right) \sum_{\lambda \in \mathbb{G T}_{N}} \frac{c_{\lambda, \mu} M_{N}(\lambda)}{P_{\lambda}\left(1, t, \ldots, t^{N-1}\right)},
\end{aligned}
$$

where the interchange in the order of summation follows from the absolute convergence of all the sums involved. (For the absolute convergence, the nonnegativity of all coefficients $c_{\lambda, \mu}$ is needed.) From the expansion above, we can extract the coefficient of $x_{1}^{\kappa_{1}} \cdots x_{N}^{\kappa_{N}}$ in the Fourier series, for any $\kappa=\left(\kappa_{1} \geq \cdots \geq \kappa_{N}\right) \in \mathbb{G}_{N}$. In fact, such term appears only in $m_{\kappa}\left(x_{1}, \ldots, x_{N} t^{N-1}\right)$ with coefficient $t^{n(\kappa)}$, where $n(\kappa)=\kappa_{2}+2 \kappa_{3}+\cdots+(N-1) \kappa_{N}$. Thus the Fourier coefficient of $x_{1}^{\kappa_{1}} \cdots x_{N}^{\kappa_{N}}$ in the expansion of $\mathcal{P}_{M_{N}}\left(x_{1}, \ldots, x_{N}\right)$ is

$$
f_{\kappa_{1}, \ldots, \kappa_{N}}=t^{n(\kappa)} \sum_{\lambda \in \mathbb{G T}_{N}} \frac{c_{\lambda, \kappa} M_{N}(\lambda)}{P_{\lambda}\left(1, t, \ldots, t^{N-1}\right)} .
$$

If $M_{N}$ is supported on $\mathbb{G T}_{N}^{+}$, then the sum defining $f_{\kappa_{1}, \ldots, \kappa_{N}}$ above is finite. Indeed the only signatures with a nonzero contribution are $\lambda \in \mathbb{G T}_{N}^{+}$with $|\lambda|=|\kappa|$. But then $|\kappa|=|\lambda| \geq \lambda_{1}$, and there are finitely many signatures $\lambda \in \mathbb{G T}_{N}$ with $|\kappa| \geq \lambda_{1} \geq \cdots \geq \lambda_{N} \geq 0$. This observation will be put to use several times.

Lemma 6.7. Let $M_{N}, M_{N}^{\prime}$ be probability measures on $\mathbb{G T}_{N}$ that are supported on $\mathbb{G T}_{N}^{+}$. If

$$
\mathcal{P}_{M_{N}}\left(x_{1}, \ldots, x_{N}\right)=\mathcal{P}_{M_{N}^{\prime}}\left(x_{1}, \ldots, x_{N}\right) \quad \forall\left(x_{1}, \ldots, x_{N}\right) \in \mathbb{T}^{N}
$$

then $M_{N}=M_{N}^{\prime}$.

Proof. Both $\mathcal{P}_{M_{N}}\left(x_{1}, \ldots, x_{N}\right)$ and $\mathcal{P}_{M_{N}^{\prime}}\left(x_{1}, \ldots, x_{N}\right)$ belong to $L^{2}\left(\mathbb{T}^{N}\right)$. The equality of these functions implies that their Fourier coefficients agree. From (6.6), this means

$$
\sum_{\mu \in \mathbb{G}_{N}^{+}} \frac{c_{\mu, \kappa} M_{N}(\mu)}{P_{\mu}\left(1, t, \ldots, t^{N-1}\right)}=\sum_{\mu \in \mathbb{G T}_{N}^{+}} \frac{c_{\mu, \kappa} M_{N}^{\prime}(\mu)}{P_{\mu}\left(1, t, \ldots, t^{N-1}\right)} \quad \forall \kappa \in \mathbb{G}_{N} .
$$

Observe that we have restricted the sum above to $\mu \in \mathbb{G} \mathbb{T}_{N}^{+}$, because $M_{N}, M_{N}^{\prime}$ are supported on positive signatures. Let $n \in \mathbb{Z}_{\geq 0}$ be arbitrary. We show that $M_{N}(\kappa)=M_{N}^{\prime}(\kappa)$ for all $\kappa \in \mathbb{G}_{N}^{+}$ with $|\kappa|=n$.

Let $C$ be the matrix whose rows and columns are parametrized by $\lambda \in \mathbb{G}_{N}^{+}$with $|\lambda|=n$, and such that its entry $C(\kappa, \mu)$ is $c_{\mu, \kappa} / P_{\mu}\left(1, t, \ldots, t^{N-1}\right)$. Observe that $C$ is a finite and square matrix. Also let $M$ (resp. $M^{\prime}$ ) be the column vector whose entries are parametrized by $\lambda \in \mathbb{G T}_{N}^{+}$ with $|\lambda|=n$ and whose entry $\mu$ is $M_{N}(\mu)$ (resp. $M_{N}^{\prime}(\mu)$ ). Then (6.7) yields $C M=C M^{\prime}$. The 
matrix $C$ is upper-triangular with respect to the order $\geq$ on signatures because $c_{\mu, \kappa}=0$ unless $\mu \geq \kappa$. Moreover the diagonal entries are $c_{\mu, \mu} / P_{\mu}\left(1, t, \ldots, t^{N-1}\right)=1 / P_{\mu}\left(1, t, \ldots, t^{N-1}\right) \neq 0$. It follows that $C$ has an inverse and $M=C^{-1} C M=C^{-1} C M^{\prime}=M^{\prime}$. Therefore $M_{N}(\kappa)=M_{N}^{\prime}(\kappa)$ $\forall \kappa \in \mathbb{G}_{N}^{+}$with $|\kappa|=n$. Since $n \in \mathbb{Z}_{\geq 0}$ was arbitrary and both $M_{N}, M_{N}^{\prime}$ are supported on $\mathbb{G T}_{N}^{+}$, we conclude $M_{N}=M_{N}^{\prime}$.

Proposition 6.8. If the sequence $\left\{M_{N}\right\}_{N \geq 0}$ (resp. finite sequence $\left\{M_{N}\right\}_{N=0,1, \ldots, k}$ ) is a $(q, t)$ coherent sequence, then

$$
\mathcal{P}_{M_{N}}\left(x_{1}, \ldots, x_{N}\right)=\mathcal{P}_{M_{N+1}}\left(1, x_{1}, \ldots, x_{N}\right), \quad \forall\left(x_{1}, \ldots, x_{N}\right) \in \mathbb{T}^{N}
$$

for all $N \geq 0$ (resp. for all $N=0,1, \ldots, k-1)$. The converse statement holds if, for each $N \geq 0$ (resp. $N=0,1, \ldots, k-1), M_{N}$ is supported on $\mathbb{G T}_{N}^{+}$.

Proof. Let us prove the first part. Let $\left\{M_{N}\right\}_{N \geq 0}$ be a $(q, t)$-coherent sequence. By making use of the branching rule, Theorem 2.5, the fact that $P_{\mu}$ is homogeneous of degree $|\mu|$, and making a change in the order of summation, we obtain

$$
\begin{aligned}
\mathcal{P}_{M_{N+1}}\left(1, x_{1}, \ldots, x_{N}\right)=\sum_{\lambda \in \mathbb{G T}_{N+1}} M_{N+1}(\lambda) \frac{P_{\lambda}\left(1, x_{1} t, \ldots, x_{N} t^{N}\right)}{P_{\lambda}\left(1, t, \ldots, t^{N}\right)} \\
=\sum_{\lambda \in \mathbb{G T}_{N+1}} \frac{M_{N+1}(\lambda)}{P_{\lambda}\left(1, t, \ldots, t^{N}\right)} \sum_{\mu \in \mathbb{G T}_{N}} \psi_{\lambda / \mu}(q, t) P_{\mu}\left(x_{1} t, \ldots, x_{N} t^{N}\right) \\
=\sum_{\lambda \in \mathbb{G T}_{N+1}} \frac{M_{N+1}(\lambda)}{P_{\lambda}\left(1, t, \ldots, t^{N}\right)} \sum_{\mu \in \mathbb{G T}_{N}} t^{|\mu|} \psi_{\lambda / \mu}(q, t) P_{\mu}\left(x_{1}, \ldots, x_{N} t^{N-1}\right) \\
=\sum_{\mu \in \mathbb{G T}_{N}} \frac{P_{\mu}\left(x_{1}, \ldots, x_{N} t^{N-1}\right)}{P_{\mu}\left(1, t, \ldots, t^{N-1}\right)} \sum_{\lambda \in \mathbb{G T}_{N+1}} t^{|\mu|} \psi_{\lambda / \mu}(q, t) \frac{P_{\mu}\left(1, t, \ldots, t^{N-1}\right)}{P_{\lambda}\left(1, t, \ldots, t^{N}\right)} M_{N+1}(\lambda) \\
=\sum_{\mu \in \mathbb{G T}_{N}} \frac{P_{\mu}\left(x_{1}, \ldots, x_{N} t^{N-1}\right)}{P_{\mu}\left(1, t, \ldots, t^{N-1}\right)} M_{N}(\mu)=\mathcal{P}_{M_{N}}\left(x_{1}, \ldots, x_{N}\right) .
\end{aligned}
$$

We can easily show that all sums above are absolutely convergent, so the change in the order of summations can be justified.

Next we prove the converse statement. Assume that $M_{N}, M_{N+1}$ are probability measures on $\mathbb{G T}_{N}, \mathbb{G T}_{N+1}$. Assume that they are supported on $\mathbb{G T}_{N}^{+}, \mathbb{G T}_{N+1}^{+}$, respectively, and also that $\mathcal{P}_{M_{N}}\left(x_{1}, \ldots, x_{N}\right)=\mathcal{P}_{M_{N+1}}\left(1, x_{1}, \ldots, x_{N}\right)$ on $\mathbb{T}^{N}$. Let $M_{N}^{\prime}$ be the measure on $\mathbb{G}_{N}$ defined by

$$
M_{N}^{\prime}(\mu)=\sum_{\lambda \in \mathbb{G T}_{N+1}} M_{N+1}(\lambda) \Lambda_{N}^{N+1}(\lambda, \mu) \quad \forall \mu \in \mathbb{G T}_{N}
$$

Since $\left[\Lambda_{N}^{N+1}(\lambda, \mu)\right]$ is a stochastic matrix and $M_{N+1}$ is a probability measure on $\mathbb{G T}_{N+1}$, then $M_{N}^{\prime}$ is a probability measure on $\mathbb{G T}_{N}$. Moreover since $M_{N+1}$ is supported on $\mathbb{G T}_{N+1}^{+}$, it follows that $M_{N}^{\prime}$ is supported on $\mathbb{G T}_{N}^{+}$. In fact, if $\mu \notin \mathbb{G} \mathbb{T}_{N} \backslash \mathbb{G} \mathbb{T}_{N}^{+}$(or equivalently $\mu_{N}<0$ ), then for any $\lambda \in \mathbb{G T}_{N+1}$, either $\lambda_{N+1}<0$ in which case $M_{N+1}(\lambda)=0$, or $\lambda_{N+1} \geq 0$ in which case $\Lambda_{N}^{N+1}(\lambda, \mu)=0$.

We will be done if we showed $M_{N}=M_{N}^{\prime}$. From what we have proved in the first part of the argument, we have $\mathcal{P}_{M_{N}^{\prime}}\left(x_{1}, \ldots, x_{N}\right)=\mathcal{P}_{M_{N+1}}\left(1, x_{1}, \ldots, x_{N}\right)$ on $\mathbb{T}^{N}$. It follows that $\mathcal{P}_{M_{N}}=\mathcal{P}_{M_{N}^{\prime}}$ on $\mathbb{T}^{N}$. An application of Lemma 6.7 concludes the proof. 
Proposition 6.9. Let $N \in \mathbb{N}$ be arbitrary. If $\left\{M^{m}\right\}_{m \geq 1}, M$ are all probability measures on $\mathbb{G T}_{N}$ such that the weak convergence holds $\mathbb{P}-\lim _{m \rightarrow \infty} M^{m}=M$, then

$$
\lim _{m \rightarrow \infty} \mathcal{P}_{M^{m}}\left(x_{1}, \ldots, x_{N}\right)=\mathcal{P}_{M}\left(x_{1}, \ldots, x_{N}\right) \quad \forall\left(x_{1}, \ldots, x_{N}\right) \in \mathbb{T}^{N}
$$

The convergence above is uniform on $\mathbb{T}^{N}$.

Proof. Let $\epsilon>0$ be a very small real number. Since $M$ is a probability measure, there exists $c>0$ large enough so that

$$
M\left(\left\{\lambda \in \mathbb{G T}_{N}: c \geq \lambda_{1} \geq \lambda_{2} \geq \cdots \geq \lambda_{N} \geq-c\right\}\right)>1-\epsilon
$$

From the weak convergence $\mathbb{P}-\lim _{m \rightarrow \infty} M^{m}=M$, there exists $N_{1} \in \mathbb{N}$ so that $m>N_{1}$ implies

$$
M^{m}\left(\left\{\lambda \in \mathbb{G T}_{N}: c \geq \lambda_{1} \geq \lambda_{2} \geq \cdots \geq \lambda_{N} \geq-c\right\}\right)>1-2 \epsilon .
$$

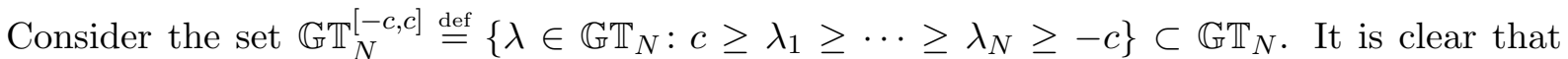
$\mathbb{G} \mathbb{T}_{N}^{[-c, c]}$ is finite and has cardinality no greater than $(2 c+1)^{N}$. Also, since $\mathbb{G T}_{N}^{[-c, c]}$ is a finite set, there exists $N_{2} \in \mathbb{N}$ such that $m>N_{2}$ implies

$$
\left|M(\lambda)-M^{m}(\lambda)\right|<\frac{\epsilon}{(2 c+1)^{N}} \quad \forall \lambda \in \mathbb{G}_{N}^{[-c, c]}
$$

We are ready to make the desired estimate. Use (6.9), (6.10), (6.11) and the triangle inequality to argue that for any $m>\max \left\{N_{1}, N_{2}\right\}$, we have

$$
\begin{aligned}
\sup _{\left(x_{1}, \ldots, x_{N}\right) \in \mathbb{T}^{N}}\left|\mathcal{P}_{M^{m}}\left(x_{1}, \ldots, x_{N}\right)-\mathcal{P}_{M}\left(x_{1}, \ldots, x_{N}\right)\right| \\
\leq \sum_{\lambda \in \mathbb{G T}_{N}^{[-c, c]}}\left|M^{m}(\lambda)-M(\lambda)\right| \sup _{\left(x_{1}, \ldots, x_{N}\right) \in \mathbb{T}^{N}}\left|\frac{P_{\lambda}\left(x_{1}, x_{2} t, \ldots, x_{N} t^{N-1}\right)}{P_{\lambda}\left(1, t, \ldots, t^{N-1}\right)}\right| \\
\quad+\sum_{\lambda \in \mathbb{G T}_{N} \backslash \mathbb{G T}_{N}^{[-c, c]}}\left(M^{m}(\lambda)+M(\lambda)\right) \sup _{\left(x_{1}, \ldots, x_{N}\right) \in \mathbb{T}^{N}}\left|\frac{P_{\lambda}\left(x_{1}, x_{2} t, \ldots, x_{N} t^{N-1}\right)}{P_{\lambda}\left(1, t, \ldots, t^{N-1}\right)}\right| \\
\leq \sum_{\lambda \in \mathbb{G T}_{N}^{[-c, c]}}\left|M^{m}(\lambda)-M(\lambda)\right|+\sum_{\lambda \in \mathbb{G T}_{N} \backslash \mathbb{G T}_{N}^{[-c, c]}}\left(M^{m}(\lambda)+M(\lambda)\right) \\
\leq \frac{\epsilon}{(2 c+1)^{N}}\left|\mathbb{G T}_{N}^{[-c, c]}\right|+M^{m}\left(\mathbb{G T}_{N} \backslash \mathbb{G T}_{N}^{[-c, c]}\right)+M\left(\mathbb{G} \mathbb{T}_{N} \backslash \mathbb{G T}_{N}^{[-c, c]}\right) \\
\leq \epsilon+2 \epsilon+\epsilon=4 \epsilon .
\end{aligned}
$$

A partial converse to the previous proposition is Proposition 6.10 below.

Proposition 6.10. Let $\left\{M^{m}\right\}_{m \geq 1}, M$, be probability measures on $\mathbb{G T}_{N}$, supported on $\mathbb{G T}_{N}^{+}$. Assume the following convergence

$$
\lim _{m \rightarrow \infty} \mathcal{P}_{M^{m}}\left(x_{1}, \ldots, x_{N}\right)=\mathcal{P}_{M}\left(x_{1}, \ldots, x_{N}\right)
$$

holds uniformly on $\mathbb{T}^{N}$, then we have the weak convergence $\mathbb{P}-\lim _{m \rightarrow \infty} M^{m}=M$. 
Proof. All functions $\left\{\mathcal{P}_{M^{m}}\right\}_{m \geq 1}, \mathcal{P}_{M}$ belong to $L^{2}\left(\mathbb{T}^{m}\right)$. Therefore the limit $\lim _{m \rightarrow \infty} \mathcal{P}_{M^{m}}\left(x_{1}, \ldots\right.$, $\left.x_{N}\right)=\mathcal{P}_{M}\left(x_{1}, \ldots, x_{N}\right)$ implies the convergence of the Fourier coefficients. Due to (6.6), this implies that, for any $\kappa \in \mathbb{G T}_{N}$, we have

$$
\lim _{m \rightarrow \infty} \sum_{\lambda \in \mathbb{G T}_{N}^{+}} \frac{c_{\lambda, \kappa} M^{m}(\lambda)}{P_{\lambda}\left(1, t, \ldots, t^{N-1}\right)}=\sum_{\lambda \in \mathbb{G T}_{N}^{+}} \frac{c_{\lambda, \kappa} M(\lambda)}{P_{\lambda}\left(1, t, \ldots, t^{N-1}\right)} .
$$

We show that $\lim _{m \rightarrow \infty} M^{m}(\lambda)=M(\lambda)$ for any $\lambda \in \mathbb{G T}_{N}^{+}$. In fact, let $n \in \mathbb{Z}_{\geq 0}$ be arbitrary and let us prove $\lim _{m \rightarrow \infty} M^{m}(\lambda)=M(\lambda)$ for any $\lambda \in \mathbb{G T}_{N}^{+}$with $|\lambda|=n$. Consider the finite, square matrix $C$ whose rows and columns are parametrized by $\lambda \in \mathbb{G T}_{N}^{+},|\lambda|=n$, and whose entries are $C(\kappa, \lambda)=c_{\lambda, \kappa} / P_{\lambda}\left(1, t, \ldots, t^{N-1}\right)$. Also let $\left\{M^{m}\right\}_{m \geq 0}, M$ be column vectors whose entries are parametrized by $\lambda \in \mathbb{G T}_{N}^{+}$with $|\lambda|=n$, and whose entries, at $\lambda \in \mathbb{G T}_{N}^{+}$, are $\left\{M^{m}(\lambda)\right\}_{m \geq 0}, M(\lambda)$. From the limit relation above, we have the entrywise limit of column vectors $\lim _{m \rightarrow \infty} C M^{m}=C M$. The matrix $C$ is upper triangular (with respect to the order on signatures given in Corollary 2.6) and has nonzero diagonal entries, thus it has an inverse $C^{-1}$. Each entry of the column vectors $M^{m}=\left(C^{-1} C\right) M^{m}=C^{-1}\left(C M^{m}\right)$ is a finite linear combination of entries of $C M^{m}$, and the same can be said about the entries of $C^{-1} C M=M$. Thus the entry-wise limit of column vectors $\lim _{m \rightarrow \infty} M^{m}=M$ follows.

By assumption, $M^{m}(\lambda)=M(\lambda)=0$ for any $\lambda \notin \mathbb{G T}_{N}^{+}$, so also $\lim _{m \rightarrow \infty} M^{m}(\lambda)=M(\lambda)$ in this case. Hence the weak convergence $\mathbb{P}-\lim _{m \rightarrow \infty} M^{m}=M$ is proved.

\subsection{Automorphisms $A_{k}$}

Recall the set $\mathcal{N}$ of nonincreasing integer sequences, given in Definition 5.1. Equip $\mathcal{N}$ with the topology of pointwise convergence. We denote a generic element of $\mathcal{N}$ by $\nu=\left(\nu_{1} \leq \nu_{2} \leq \cdots\right)$. For each $k \in \mathbb{Z}$, we can define the continuous map $A_{k}: \mathcal{N} \rightarrow \mathcal{N}$ by $\nu \mapsto A_{k} \nu=\left(\nu_{1}+k \leq \nu_{2}+k \leq \cdots\right)$. It is clear that $A_{k}$ has inverse $A_{-k}$, so each $A_{k}$ is a homeomorphism.

Similar automorphisms can be constructed for $\mathbb{G T}$ and $\mathcal{T}$. In detail, we can define the map $A_{k}: \mathbb{G T} \rightarrow \mathbb{G T}$ by $\lambda \mapsto A_{k} \lambda=\left(\lambda_{1}+k \geq \lambda_{2}+k \geq \cdots\right), A_{k} \varnothing=\varnothing$, whose inverse is $A_{-k}$, and moreover it restricts to automorphisms $\mathbb{G T}_{N} \rightarrow \mathbb{G}_{N}$ for each $N \in \mathbb{Z}_{\geq 0}$. It is clear that $\mu \prec \lambda$ implies $A_{k} \mu \prec A_{k} \lambda$, so the automorphism $A_{k}$ of $\mathbb{G T}$ induces the automorphism of measurable spaces $A_{k}: \mathcal{T} \rightarrow \mathcal{T}, \tau=\left(\tau^{(0)} \prec \tau^{(1)} \prec \tau^{(2)} \prec \cdots\right) \mapsto\left(A_{k} \tau^{(0)} \prec A_{k} \tau^{(1)} \prec A_{k} \tau^{(2)} \prec \cdots\right)$.

The same notation $A_{k}$ is used to define automorphisms of the spaces $\mathcal{N}, \mathbb{G} \mathbb{T}$ and $\mathcal{T}$, but there should be no confusion each time it is used in the future.

We have introduced the automorphisms $A_{k}$ because, in Lemma 6.12 below, we will relate the extreme central probability measures associated to $\nu$ and $A_{k} \nu$. The starting point is the following simple statement, which has nothing to do with probability.

Lemma 6.11. Recall the functions $\Phi^{\nu}\left(x_{1}, \ldots, x_{m} ; q, t\right)$, defined in the statement of Theorem 5.5. Let $\nu \in \mathcal{N}$ and $k \in \mathbb{Z}$ be arbitrary. The following equality holds

$$
\begin{aligned}
& \Phi^{A_{k} \nu}\left(x_{1}, \ldots, x_{m} ; q, t\right)=t^{k\left(\begin{array}{c}
m \\
2
\end{array}\right)}\left(x_{1} \cdots x_{m}\right)^{k} \Phi^{\nu}\left(x_{1}, \ldots, x_{m} ; q, t\right), \\
& \forall\left(x_{1}, \ldots, x_{m}\right) \in(\mathbb{C} \backslash\{0\})^{m} .
\end{aligned}
$$

Proof. As both sides of the identity (6.13) are analytic functions on $(\mathbb{C} \backslash\{0\})^{m}$, we only need to prove the equality for $\left(x_{1}, \ldots, x_{m}\right) \in \mathcal{U}_{m}$, where the domain $\mathcal{U}_{m}$ was defined in the statement of Theorem 5.5. We can now make use of formula (5.8) for $\Phi^{\nu}\left(x_{1}, \ldots, x_{m} ; q, t\right)$. Observe that the only place where $\nu$ appears in the right-hand side is inside the univariate functions $\Phi^{\nu}\left(x_{i} ; q, t\right)$. 
The operator $\widetilde{D}_{q, \theta}^{(m)}$ satisfies that for any Laurent polynomial $f$ on variables $x_{1}, \ldots, x_{m}$, the following identity holds

$$
\widetilde{D}_{q, \theta}^{(m)}\left\{\left(x_{1} \cdots x_{m}\right)^{k} f\right\}=t^{k\left(\begin{array}{c}
m \\
2
\end{array}\right)}\left(x_{1} \cdots x_{m}\right)^{k} \widetilde{D}_{q, \theta}^{(m)}\{f\} .
$$

We deduce that the lemma will be proved for all $m \in \mathbb{N}$ once we prove it for $m=1$, that is, we need $\Phi^{A_{k} \nu}(x ; q, t)=x^{k} \Phi^{\nu}(x ; q, t) \forall x \in \mathcal{U}=\bigcap_{k \geq 1}\left\{x \neq q^{-k}\right\} \cap\{x \neq 0\}$. The latter statement easily follows from the integral definition of $\Phi^{\nu}(x ; q, t)$ in Theorem 5.2 (to be precise, our desired statement follows after a change of variables $z \mapsto z+k$ in the integral).

Next we introduce new maps $\left\{A_{k}\right\}_{k \in \mathbb{Z}}$ on spaces of probability measures.

For a probability measure $M_{m}$ on $\mathbb{G T}_{m}$, define $A_{k} M_{m}$ as the pushforward of $M_{m}$ under the automorphism $A_{k}$ of $\mathbb{G T}_{m}$, i.e., $A_{k} M_{m}(\mu) \stackrel{\text { def }}{=} M_{m}\left(A_{-k} \mu\right)$ for all $\mu \in \mathbb{G T}_{m}$. Observe that if we let $\delta_{\lambda}$ be the probability measure on $\mathbb{G}_{m}$ given by the delta mass at $\lambda \in \mathbb{G T}_{m}$, then $A_{k} \delta_{\lambda}=\delta_{A_{k} \lambda}$ for any $k \in \mathbb{Z}$.

Similarly if $M$ is a probability measure on $\mathcal{T}$, define $A_{k} M$ as the pushforward of $M$ under the automorphism $A_{k}$ of $\mathcal{T}$. This can be described concretely as follows. The automorphism $A_{k}$ of $\mathcal{T}$ induces automorphisms $A_{k}$ on the set of paths of length $m$ in the GT graph, for any $m \in \mathbb{N}$ :

$$
\phi=\left(\phi^{(0)} \prec \phi^{(1)} \prec \cdots \prec \phi^{(m)}\right) \mapsto A_{k} \phi \stackrel{\text { def }}{=}\left(A_{k} \phi^{(0)} \prec A_{k} \phi^{(1)} \prec \cdots \prec A_{k} \phi^{(m)}\right) \text {. }
$$

It is therefore natural to define also an automorphism on the family of cylinder sets by $A_{k} S_{\phi} \stackrel{\text { def }}{=}$ $S_{A_{k} \phi}$. Then $A_{k} M$ is given by $A_{k} M\left(S_{\phi}\right) \stackrel{\text { def }}{=} M\left(A_{-k} S_{\phi}\right)=M\left(S_{A_{-k} \phi}\right)$ for all finite paths $\phi=$ $\left(\phi^{(0)} \prec \phi^{(1)} \prec \cdots \prec \phi^{(m)}\right)$.

Lemma 6.12. Let $M \in M_{\text {prob }}(\mathcal{T})$ be a $(q, t)$-central probability measure and $\left\{M_{m}\right\}_{m \geq 0}$ be its associated $(q, t)$-coherent sequence. Then also $A_{k} M \in M_{\operatorname{prob}}(\mathcal{T})$ and its associated $(q, t)$-coherent sequence is $\left\{A_{k} M_{m}\right\}_{m \geq 0}$.

Proof. Clearly $A_{k} M \in M_{\text {prob }}(\mathcal{T})$ is a consequence of the definition of $A_{k} M$ as a pushforward of the probability measure $M$. The second claim can be restated as

$$
\left(\operatorname{Proj}_{m}\right)_{*} A_{k} M=A_{k} M_{m}
$$

This equality follows from the definitions of $A_{k} M$ and $A_{k} M_{m}$ as pushforwards of $M$ and $M_{m}$, the fact that $\left(\operatorname{Proj}_{m}\right)_{*} M=M_{m}$, and the evident commutativity of the diagram

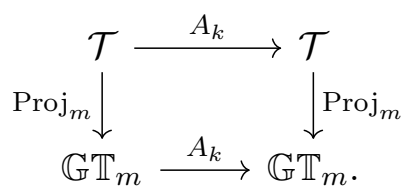

\section{The boundary of the $(q, t)$-Gelfand-Tsetlin graph}

In this section we prove Theorem 1.3, which characterizes the (minimal) boundary of the $(q, t)$-GT graph. Along the way, we also define and characterize the Martin boundary of the $(q, t)-\mathrm{GT}$ graph.

Assume throughout this section that $q \in(0,1), \theta \in \mathbb{N}$ and set $t=q^{\theta}$. Recall the notation $\mathbb{P}-\lim _{k \rightarrow \infty} M_{k}=M$ indicates that a sequence of probability measures $\left\{M_{k}\right\}_{k \geq 1}$ converges weakly to $M$. 


\subsection{The Martin boundary: definition and preliminaries}

For any $\lambda \in \mathbb{G T}_{N}$, let $\delta_{\lambda}$ be the delta mass at $\lambda$. As remarked in Section 6.1, there exists a unique $(q, t)$-coherent sequence $\left\{M_{m}^{\lambda}\right\}_{m=0,1, \ldots, N}$ such that each $M_{m}^{\lambda}$ is a probability measure on $\mathbb{G T}_{m}$ and $M_{N}^{\lambda}=\delta_{\lambda}$. Such a sequence is given explicitly by $M_{N}^{\lambda}=\delta_{\lambda}$ and $M_{m}^{\lambda}=\Lambda_{m}^{N} \delta_{\lambda} \forall 0 \leq m \leq N-1$, where the probability measures $\Lambda_{m}^{N} \delta_{\lambda}$ on $\mathbb{G T}_{m}$ are given explicitly in (6.4). Moreover recall that for a $(q, t)$-central probability measure $M$ on $\mathcal{T}$, we can associate a $(q, t)$-coherent sequence $\left\{M_{m}\right\}_{m \geq 0}$ as in Definition 6.4.

Definition 7.1. The Martin boundary of the $(q, t)$-Gelfand-Tsetlin graph is the subset of $(q, t)$-central probability measures $M \in M_{\text {prob }}(\mathcal{T})$ for which there exists a sequence $\{\lambda(N)\}_{N \geq 1}$, $\lambda(N) \in \mathbb{G T}_{N}$, such that the following weak limits hold

$$
\mathbb{P}-\lim _{N \rightarrow \infty} \Lambda_{m}^{N} \delta_{\lambda(N)}=M_{m} \quad \forall m=0,1,2, \ldots
$$

Let us denote the Martin boundary by $\Omega_{q, t}^{\mathrm{Martin}} \subset M_{\text {prob }}(\mathcal{T})$ and equip it with its subspace topology, namely with the topology of weak convergence.

To characterize the Martin boundary $\Omega_{q, t}^{\mathrm{Martin}}$, we begin by giving necessary conditions on sequences of signatures $\{\lambda(N)\}_{N \geq 1}$ which yield a weak convergence as above. In this section, we sometimes denote $M_{N}^{\lambda} \stackrel{\text { def }}{=} \delta_{\lambda}$ the delta mass at $\lambda \in \mathbb{G T}_{N}$, and $\left\{M_{m}^{\lambda}=\Lambda_{m}^{N} \delta_{\lambda}\right\}_{m=0,1, \ldots, N}$ the corresponding $(q, t)$-coherent sequence.

Lemma 7.2. Assume that $\{\lambda(N)\}_{N \geq 1}, \lambda(N) \in \mathbb{G}_{N}$, is a sequence of signatures such that $\left\{\Lambda_{1}^{N} \delta_{\lambda(N)}\right\}_{N \geq 1}$ converges weakly, as $N \rightarrow \infty$, to some probability measure $\mathfrak{m}$ on $\mathbb{G}_{1}=\mathbb{Z}$. Then the sequence $\left\{\lambda(N)_{N}\right\}_{N \geq 1}$ is bounded.

Proof. We use the Macdonald generating functions of Section 6.3 above. By Proposition 6.8, we have

$$
\mathcal{P}_{\delta_{\lambda(N)}}\left(1^{N-1}, z\right)=\mathcal{P}_{M_{1}^{\lambda(N)}}(z)
$$

and then

$$
\frac{P_{\lambda(N)}\left(1, t, \ldots, t^{N-2}, t^{N-1} z\right)}{P_{\lambda(N)}\left(1, t, \ldots, t^{N-2}, t^{N-1}\right)}=\frac{P_{\lambda(N)}\left(z, t^{-1}, t^{-2}, \ldots, t^{1-N}\right)}{P_{\lambda(N)}\left(1, t^{-1}, t^{-2}, \ldots, t^{1-N}\right)}=\sum_{n \in \mathbb{Z}} M_{1}^{\lambda(N)}(n) z^{n} .
$$

In the sum of the right-hand side above, $n$ ranges from $\lambda(N)_{N}$ to $\lambda(N)_{1}$ because of the branching rule for Macdonald polynomials. We multiply the equality by $z^{-\lambda(N)_{N}}$ and then set $z=0$, so in the right-hand side one clearly picks up the coefficient of $z^{\lambda(N)_{N}}$, namely $\left.M_{1}^{\lambda(N)}(\lambda(N))_{N}\right)$. By the index stability of Macdonald polynomials, 2.2), the left-hand side is

$$
\begin{aligned}
z^{-\lambda(N)_{N}} & \left.\frac{P_{\lambda(N)}\left(z, t^{-1}, \ldots, t^{1-N}\right)}{P_{\lambda(N)}\left(1, t^{-1}, \ldots, t^{1-N}\right)}\right|_{z=0} \\
= & \left.\frac{\left(z \cdot t^{-1} \cdots t^{1-N}\right)^{-\lambda(N)_{N}}}{\left(1 \cdot t^{-1} \cdots t^{1-N}\right)^{-\lambda(N)_{N}}} \frac{P_{\lambda(N)}\left(z, t^{-1}, \ldots, t^{1-N}\right)}{P_{\lambda(N)}\left(1, t^{-1}, \ldots, t^{1-N}\right)}\right|_{z=0} \\
= & \left.\frac{P_{\left(\lambda(N)_{1}-\lambda(N)_{N}, \ldots, \lambda(N)_{N-1}-\lambda(N)_{N}, 0\right)}\left(z, t^{-1}, \ldots, t^{1-N}\right)}{P_{\left(\lambda(N)_{1}-\lambda(N)_{N}, \ldots, \lambda(N)_{N-1}-\lambda(N)_{N}, 0\right)}\left(1, t^{-1}, \ldots, t^{1-N}\right)}\right|_{z=0} \\
= & \frac{P_{\left(\lambda(N)_{1}-\lambda(N)_{N}, \ldots, \lambda(N)_{N-1}-\lambda(N)_{N}\right)}\left(t^{-1}, \ldots, t^{1-N}\right)}{P_{\left(\lambda(N)_{1}-\lambda(N)_{N}, \ldots, \lambda(N)_{N-1}-\lambda(N)_{N}, 0\right)}\left(1, t^{-1}, \ldots, t^{1-N}\right)} .
\end{aligned}
$$


Thanks to Theorem 2.3, we can then obtain a lower bound for $M_{1}^{\lambda(N)}\left(\lambda(N)_{N}\right)$ as follows:

$$
\begin{aligned}
M_{1}^{\lambda(N)}\left(\lambda(N)_{N}\right)=\frac{P_{\left(\lambda(N)_{1}-\lambda(N)_{N}, \ldots, \lambda(N)_{N-1}-\lambda(N)_{N}\right)}\left(t^{-1}, \ldots, t^{1-N}\right)}{P_{\left(\lambda(N)_{1}-\lambda(N)_{N}, \ldots, \lambda(N)_{N-1}-\lambda(N)_{N}, 0\right)}\left(1, t^{-1}, \ldots, t^{1-N}\right)} \\
=\frac{P_{\left(\lambda(N)_{1}-\lambda(N)_{N}, \ldots, \lambda(N)_{N-1}-\lambda(N)_{N}\right)}\left(t^{N-2}, \ldots, t, 1\right)}{P_{\left(\lambda(N)_{1}-\lambda(N)_{N}, \ldots, \lambda(N)_{N-1}-\lambda(N)_{N}, 0\right)}\left(t^{N-1}, \ldots, t, 1\right)} \\
=\prod_{1 \leq i<j \leq N-1} \frac{\left(q^{\lambda(N)_{i}-\lambda(N)_{j}} t^{j-i} ; q\right)_{\infty}\left(t^{j-i+1} ; q\right)_{\infty}}{\left(q^{\lambda(N)_{i}-\lambda(N)_{j} t^{j-i+1}} ; q\right)_{\infty}\left(t^{j-i} ; q\right)_{\infty}} \\
\quad \times \prod_{1 \leq i<j \leq N} \frac{\left(q^{\lambda(N)_{i}-\lambda(N)_{j}} t^{j-i+1} ; q\right)_{\infty}\left(t^{j-i} ; q\right)_{\infty}}{\left(q^{\lambda(N)_{i}-\lambda(N)_{j}} t^{j-i} ; q\right)_{\infty}\left(t^{j-i+1} ; q\right)_{\infty}} \\
=\prod_{i=1}^{N-1} \frac{\left(q^{\lambda(N)_{i}-\lambda(N)_{N}} t^{N-i+1} ; q\right)_{\infty}\left(t^{N-i} ; q\right)_{\infty}}{\left(q^{\left.\lambda(N)_{i}-\lambda(N)_{N} t^{N-i} ; q\right)_{\infty}\left(t^{N-i+1} ; q\right)_{\infty}}\right.} \\
=\prod_{i=1}^{N-1} \frac{\left(1-t^{N-i}\right)\left(1-q t^{N-i}\right) \cdots\left(1-q^{\theta-1} t^{N-i}\right)}{\left(1-q^{\lambda(N)_{i}-\lambda(N)_{N} t^{N-i}}\right) \cdots\left(1-q^{\lambda(N)_{i}-\lambda(N)_{N}+\theta-1} t^{N-i}\right)} \\
\geq \prod_{i=1}^{N-1}\left(1-t^{N-i}\right)\left(1-q t^{N-i}\right) \ldots\left(1-q^{\theta-1} t^{N-i}\right) \geq \prod_{i=1}^{N-1}\left(1-t^{N-i}\right)^{\theta} \geq\left((t ;)_{\infty}\right)^{\theta} .
\end{aligned}
$$

On the other hand, since $(t ; t)_{\infty} \in(0,1)$ and $\mathbb{P}-\lim _{N \rightarrow \infty} M_{1}^{\lambda(N)}=\mathfrak{m}$, then there exist $N_{1}, N_{2} \in \mathbb{N}$ large enough such that

$$
M_{1}^{\lambda(N)}\left(\left\{(n) \in \mathbb{G T}_{1}:-N_{1} \leq n \leq N_{1}\right\}\right)>1-\left((t ; t)_{\infty}\right)^{\theta} \quad \forall N>N_{2} .
$$

We conclude that $-N_{1} \leq \lambda(N)_{N} \leq N_{1}$, for all $N>N_{2}$. Therefore the sequence $\left\{\lambda(N)_{N}\right\}_{N \geq 1}$ is bounded.

One actually has the following more general statement.

Lemma 7.3. Let $k \in \mathbb{N}$. Assume that $\{\lambda(N)\}_{N \geq 1}, \lambda(N) \in \mathbb{G T}_{N}$, is a sequence of signatures such that the sequence $\Lambda_{k}^{N} \delta_{\lambda(N)}$ converges weakly, as $N \rightarrow \infty$, to some probability measure $\mathfrak{m}$ on $\mathbb{G T}_{k}$. Then for any $i=1, \ldots, k$, the sequence $\left\{\lambda(N)_{N-i+1}\right\}_{N \geq 1}$, is bounded.

Proof. The argument here is very similar to that of the previous proof for $k=1$. As before, by making use of Macdonald generating functions, we can derive a more general equation than (7.1), which is

$$
\begin{aligned}
& \frac{P_{\lambda(N)}\left(z_{1} t^{1-k}, \ldots, z_{k-1} t^{-1}, z_{k}, t^{-k}, \ldots, t^{1-N}\right)}{P_{\lambda(N)}\left(1, t^{-1}, \ldots, t^{1-N}\right)} \\
& =\sum_{\mu \in \mathbb{G T}_{k}} M_{k}^{\lambda(N)}(\mu) \frac{P_{\mu}\left(z_{1} t^{1-k}, \ldots, z_{k-1} t^{-1}, z_{k}\right)}{P_{\mu}\left(1, t^{-1}, \ldots, t^{1-k}\right)} .
\end{aligned}
$$

In the sum of the right-hand side above, note that $\mu$ ranges over signatures in $\mathbb{G T}_{k}$ such that

$$
\mu_{k} \geq \lambda(N)_{N}, \quad \mu_{k-1} \geq \lambda(N)_{N-1}, \quad \ldots, \quad \mu_{1} \geq \lambda(N)_{N-k+1} .
$$

This is a consequence of the branching rule for Macdonald polynomials. Another relevant observation is that for any $\mu \in \mathbb{G T}_{k}$ satisfying (7.3), any monomial $c_{m_{1}, \ldots, m_{k}} z_{1}^{m_{1}} \cdots z_{k}^{m_{k}}$ with $c_{m_{1}, \ldots, m_{k}} \neq 0$ in the expansion of $P_{\mu}\left(z_{1} t^{1-k}, \ldots, z_{k-1} t^{-1}, z_{k}\right)$ satisfies: $m_{k} \geq \lambda(N)_{N}$; if $m_{k}=$ 
$\lambda(N)_{N}$ then $m_{k-1} \geq \lambda(N)_{N-1}$; and so on until, if $m_{2}=\lambda(N)_{N-k+2}, \ldots, m_{k} \geq \lambda(N)_{N}$, then $m_{1} \geq \lambda(N)_{N-k+1}$. This is a consequence of the triangularity property of the Macdonald polynomials, see Definition/Proposition 2.1.

In equation (7.2) above, multiply both sides by $z_{k}^{-\lambda(N)_{N}}$ and then set $z_{k}=0$. From the branching rule for Macdonald polynomials and the fact that the branching coefficients satisfy

$$
\psi_{\lambda(N) /\left(\lambda(N)_{1}, \ldots, \lambda(N)_{N-1}\right)}(q, t)=1,
$$

the resulting left-hand side is

$$
\frac{P_{\left(\lambda(N)_{1}, \ldots, \lambda(N)_{N-1}\right)}\left(z_{1} t^{1-k}, \ldots, z_{k-1} t^{-1}, t^{-k}, \ldots, t^{1-N}\right)}{P_{\lambda(N)}\left(1, t^{-1}, \ldots, t^{1-N}\right)} .
$$

(Note that the argument $z_{k}$ is no longer present in the numerator.) Similarly, from the property

$$
\psi_{\mu /\left(\mu_{1}, \ldots, \mu_{k-1}\right)}(q, t)=1, \quad \text { for any } \quad \mu \in \mathbb{G T}_{k},
$$

the resulting right-hand side is

$$
\sum_{\mu \in \mathbb{G T}_{k}: \mu_{k}=\lambda(N)_{N}} M_{k}^{\lambda(N)}(\mu) \frac{P_{\left(\mu_{1}, \ldots, \mu_{k-1}\right)}\left(z_{1} t^{1-k}, \ldots, z_{k-1} t^{-1}\right)}{P_{\mu}\left(1, t^{-1}, \ldots, t^{1-k}\right)} .
$$

After that, multiply both sides by $\left(z_{k-1} t^{-1}\right)^{-\lambda(N)_{N-1}}$ and then set $z_{k-1}=0$; this gives

$$
\begin{aligned}
& \frac{P_{\left(\lambda(N)_{1}, \ldots, \lambda(N)_{N-2}\right)}\left(z_{1} t^{1-k}, \ldots, z_{k-2} t^{-2}, t^{-k}, \ldots, t^{1-N}\right)}{P_{\lambda(N)}\left(1, t^{-1}, \ldots, t^{1-N}\right)} \\
& =\sum_{\substack{\mu \in \mathbb{G}_{k}: \\
\mu_{k-1}=\lambda(N)_{N-1}, \mu_{k}=\lambda(N)_{N}}} M_{k}^{\lambda(N)}(\mu) \frac{P_{\left(\mu_{1}, \ldots, \mu_{k-2}\right)}\left(z_{1} t^{1-k}, \ldots, z_{k-2} t^{-2}\right)}{P_{\mu}\left(1, t^{-1}, \ldots, t^{1-k}\right)} .
\end{aligned}
$$

Repeat the same procedure $k$ times, until we have multiplied both sides by $\left(z_{1} t^{1-k}\right)^{-\lambda(N)_{N-k+1}}$ and set $z_{1}=0$. The end result is

$$
\begin{aligned}
& M_{k}^{\lambda(N)}\left(\lambda(N)_{N-k+1}, \ldots, \lambda(N)_{N}\right) \\
& =\frac{P_{\left(\lambda(N)_{1}, \ldots, \lambda(N)_{N-k}\right)}\left(t^{-k}, \ldots, t^{1-N}\right) P_{\left(\lambda(N)_{N-k+1}, \ldots, \lambda(N)_{N}\right)}\left(1, t^{-1}, \ldots, t^{1-k}\right)}{P_{\lambda(N)}\left(1, t^{-1}, \ldots, t^{1-N}\right)} \\
& =t^{(N-k)\left(\lambda(N)_{N-k+1}+\lambda(N)_{N-k+2}+\cdots+\lambda(N)_{N}\right)} \\
& \times \frac{P_{\left(\lambda(N)_{1}, \ldots, \lambda(N)_{N-k}\right)}\left(1, t, \ldots, t^{N-k-1}\right) P_{\left(\lambda(N)_{N-k+1}, \ldots, \lambda(N)_{N}\right)}\left(1, t, \ldots, t^{k-1}\right)}{P_{\lambda(N)}\left(1, t, t^{2}, \ldots, t^{N-1}\right)} \\
& =\prod_{i=1}^{N-k} \prod_{j=N-k+1}^{N} \frac{\left(1-t^{j-i}\right)\left(1-q t^{j-i}\right) \cdots\left(1-q^{\theta-1} t^{j-i}\right)}{\left(1-q^{\lambda(N)_{i}-\lambda(N)_{j} t^{j-i}}\right)\left(1-q^{\lambda(N)_{i}-\lambda(N)_{j}+1} t^{j-i}\right) \cdots\left(1-q^{\lambda(N)_{i}-\lambda(N)_{j}+\theta-1} t^{j-i}\right)} \\
& \geq \prod_{i=1}^{N-k} \prod_{j=N-k+1}^{N}\left(1-t^{j-i}\right)\left(1-q t^{j-i}\right) \cdots\left(1-q^{\theta-1} t^{j-i}\right) \geq\left((t ; t)_{\infty}\right)^{\theta k},
\end{aligned}
$$

where we used the homogeneity of Macdonald polynomials for the second equality, as well as three applications of Theorem 2.3 for the third equality. Then $M_{k}^{\lambda(N)}\left(\lambda(N)_{N-k+1}, \ldots, \lambda(N)_{N}\right) \geq c_{k}=$ $\left((t ; t)_{\infty}\right)^{\theta k}>0$. On the other hand, since $M_{k}^{\lambda(N)}$ converges weakly to $\mathfrak{m}$, there exist $N_{1}, N_{2} \in \mathbb{N}$ large enough such that

$$
M_{k}^{\lambda(N)}\left(\left\{\left(n_{1}, \ldots, n_{k}\right) \in \mathbb{G T}_{k}:-N_{1} \leq n_{1}, \ldots, n_{k} \leq N_{1}\right\}\right)>1-c_{k} \quad \forall N>N_{2} .
$$


Therefore we conclude that $-N_{1} \leq \lambda(N)_{N-k+1}, \ldots, \lambda(N)_{N} \leq N_{1}$ for all $N>N_{2}$. We conclude that each sequence $\left\{\lambda(N)_{N-i+1}\right\}_{N \geq 1}, i=1, \ldots, k$, is uniformly bounded by a constant.

Next we show that $\Omega_{q, t}^{\text {Martin }}$ is in bijection with the set $\mathcal{N}$. We do so by first constructing a map $\Omega_{q, t}^{\mathrm{Martin}} \rightarrow \mathcal{N}$ that will later be shown to be bijective.

Proposition 7.4. Let $M$ be a $(q, t)$-central probability measure on $\mathcal{T}$ and $\left\{M_{m}\right\}_{m=0,1,2, \ldots}$ be its associated $(q, t)$-coherent system. If $M$ belongs to $\Omega_{q, t}^{\mathrm{Martin}}$, then there exists a unique $\nu \in \mathcal{N}$ such that

$$
\begin{aligned}
& \Phi^{\nu}\left(x_{1} t^{1-m}, \ldots, x_{m-1} t^{-1}, x_{m} ; q, t\right)=\mathcal{P}_{M_{m}}\left(x_{1}, \ldots, x_{m}\right), \\
& \forall\left(x_{1}, \ldots, x_{m}\right) \in \mathbb{T}^{m}, \quad \forall m \geq 1 .
\end{aligned}
$$

The function $\Phi^{\nu}\left(z_{1}, \ldots, z_{m} ; q, t\right)$ was defined in Theorem 5.2 for $m=1$, and in Theorem 5.5 for general $m$.

Proof. Let $M \in \Omega_{q, t}^{\mathrm{Martin}}$. By definition, there exists a sequence $\{\lambda(N)\}_{N \geq 1}$ such that $\lambda(N) \in$ $\mathbb{G T}_{N}, N \geq 1$, and $\mathbb{P}-\lim _{m \rightarrow \infty} \Lambda_{m}^{N} \delta_{\lambda(N)}=M_{m}$ holds weakly, for any $m \in \mathbb{N}$.

By Lemma 7.3, it follows that for any $i=1,2, \ldots$, the sequence $\left\{\lambda(N)_{N-i+1}\right\}_{N \geq 1}$ is uniformly bounded. In particular, since $\left\{\lambda(N)_{N}\right\}_{N \geq 1}$ is uniformly bounded, there exists a subsequence $\left\{N_{1}^{1}<N_{2}^{1}<N_{3}^{1}<\cdots\right\} \subset \mathbb{N}$ such that $\lambda\left(N_{1}^{1}\right)_{N_{1}^{1}}=\lambda\left(N_{2}^{1}\right)_{N_{2}^{1}}=\cdots$. Analogously, using that $\left\{\lambda(N)_{N-1}\right\}_{N \geq 1}$ is bounded, there exists a subsequence $\left\{N_{1}^{2}<N_{2}^{2}<N_{3}^{2}<\cdots\right\} \subset\left\{N_{1}^{1}<\right.$ $\left.N_{2}^{1}<N_{3}^{1}<\cdots\right\}$ such that $\lambda\left(N_{1}^{2}\right)_{N_{1}^{2}-1}=\lambda\left(N_{2}^{2}\right)_{N_{2}^{2}-1}=\cdots$. In a similar fashion, we can define subsequences $\left\{N_{1}^{k}<N_{2}^{k}<\cdots\right\}$ inductively.

Now consider the subsequence $\left\{N_{1}=N_{1}^{1}<N_{2}=N_{2}^{2}<N_{3}=N_{3}^{3}<\cdots\right\} \subset \mathbb{N}$. By construction, the sequence $\left\{\lambda\left(N_{k}\right)\right\}_{k \geq 1}$ is such that the limits $\lim _{k \rightarrow \infty} \lambda\left(N_{k}\right)_{N_{k}-i+1}$ exist for all $i=1,2, \ldots$. Consequently, there exists $\nu \in \mathcal{N}$ such that $\left\{\lambda\left(N_{k}\right)\right\}_{k \geq 1}$ stabilizes to $\nu$ in the sense of Definition 5.1. From Theorem 5.5, the following limit

$$
\begin{aligned}
\lim _{k \rightarrow \infty} & \frac{P_{\lambda\left(N_{k}\right)}\left(x_{1} t^{1-m}, x_{2} t^{2-m}, \ldots, x_{m}, t^{-m}, \ldots, t^{1-N_{k}}\right)}{P_{\lambda\left(N_{k}\right)}\left(1, t^{-1}, \ldots, t^{1-N_{k}}\right)} \\
& =\Phi^{\nu}\left(x_{1} t^{1-m}, x_{2} t^{2-m}, \ldots, x_{m} ; q, t\right)
\end{aligned}
$$

holds uniformly for $\left(x_{1}, \ldots, x_{m}\right)$ in compact subsets of the domain $(\mathbb{C} \backslash\{0\})^{m}$, in particular the convergence is uniform on $\mathbb{T}^{m}$.

On the other hand, since we also have the weak convergence $\mathbb{P}-\lim _{k \rightarrow \infty} \Lambda_{m}^{N_{k}} \delta_{\lambda\left(N_{k}\right)}=M_{m}$, then by Proposition 6.9:

$$
\lim _{k \rightarrow \infty} \mathcal{P}_{\Lambda_{m}^{N_{k}} \delta_{\lambda\left(N_{k}\right)}}\left(x_{1}, \ldots, x_{m}\right)=\mathcal{P}_{M_{m}}\left(x_{1}, \ldots, x_{m}\right)
$$

uniformly on $\mathbb{T}^{m}$. By Proposition 6.8 and the homogeneity of the Macdonald polynomials, the Macdonald generating function of $\Lambda_{m}^{N_{k}} \delta_{\lambda\left(N_{k}\right)}$ equals

$$
\begin{array}{r}
\mathcal{P}_{\Lambda_{m}^{N_{k}} \delta_{\lambda\left(N_{k}\right)}}\left(x_{1}, \ldots, x_{m}\right)=\mathcal{P}_{\delta_{\lambda\left(N_{k}\right)}}\left(1^{N_{k}-m}, x_{1}, \ldots, x_{m}\right) \\
=\frac{P_{\lambda\left(N_{k}\right)}\left(1, t, \ldots, t^{N_{k}-m-1}, t^{N_{k}-m} x_{1}, \ldots, t^{N_{k}-1} x_{m}\right)}{P_{\lambda\left(N_{k}\right)}\left(1, t, t^{2}, \ldots, t^{N_{k}-1}\right)} \\
=\frac{P_{\lambda\left(N_{k}\right)}\left(x_{1} t^{1-m}, x_{2} t^{2-m}, \ldots, x_{m}, t^{-m}, \ldots, t^{1-N_{k}}\right)}{P_{\lambda\left(N_{k}\right)}\left(1, t^{-1}, t^{-2}, \ldots, t^{1-N_{k}}\right)} .
\end{array}
$$


By combining (7.5), (7.6) and (7.7), we conclude

$$
\Phi^{\nu}\left(x_{1} t^{1-m}, x_{2} t^{2-m}, \ldots, x_{m} ; q, t\right)=\mathcal{P}_{M_{m}}\left(x_{1}, \ldots, x_{m}\right) \quad \text { for all } \quad\left(x_{1}, \ldots, x_{m}\right) \in \mathbb{T}^{m}
$$

and all $m \geq 1$, as desired. The statement about the uniqueness of $\nu \in \mathcal{N}$ follows from Lemma 5.4.

Because of Proposition 7.4, for each $M \in \Omega_{q, t}^{\mathrm{Martin}}$, there exists a unique $\nu \in \mathcal{N}$ such that equation (7.4) is satisfied for all $m \in \mathbb{N}$. Thus there is a well-defined map of sets

$$
\begin{aligned}
\mathbf{N}: & \Omega_{q, t}^{\text {Martin }} \rightarrow \mathcal{N}, \\
& M \mapsto \nu,
\end{aligned}
$$

which is determined by setting $\nu=\mathbf{N}(M)$ be the unique element of $\mathcal{N}$ such that (7.4) is satisfied. We prove below that $\mathbf{N}$ is bijective, but first we show the convenient fact that $\mathbf{N}$ commutes with the automorphisms $A_{k}, k \in \mathbb{Z}$, of Section 6.4.

Lemma 7.5. Let $M \in \Omega_{q, t}^{\mathrm{Martin}}, k \in \mathbb{Z}$. Then $A_{k} M \in \Omega_{q, t}^{\mathrm{Martin}}$ and $\mathbf{N}\left(A_{k} M\right)=A_{k} \mathbf{N}(M)$.

Proof. As $M \in \Omega_{q, t}^{\mathrm{Martin}} \subset M_{\mathrm{Prob}}(\mathcal{T})$, Lemma 6.12 shows $A_{k} M \in M_{\mathrm{Prob}}(\mathcal{T})$ and $\left\{A_{k} M_{m}\right\}_{m \geq 0}$ is its corresponding $(q, t)$-coherent sequence. By definition, there exists a sequence $\{\lambda(N)\}_{N \geq 1}$, $\lambda(N) \in \mathbb{G T}_{N}$, such that the weak convergence holds $\mathbb{P}-\lim _{N \rightarrow \infty} \Lambda_{m}^{N} \delta_{\lambda(N)}=M_{m} \forall m \geq 0$. We claim that $\mathbb{P}-\lim _{N \rightarrow \infty} \Lambda_{m}^{N} \delta_{A_{k} \lambda(N)}=A_{k} M_{m} \forall m \geq 0$, which would show $A_{k} M \in \Omega_{q, t}^{\text {Martin }}$ indeed.

Take any $m \in \mathbb{Z}_{\geq 0}, \mu \in \mathbb{G}_{m}$, then

$$
\begin{aligned}
\left(\Lambda_{m}^{N} \delta_{A_{k} \lambda(N)}\right)(\mu) & =\Lambda_{m}^{N}\left(A_{k} \lambda(N), \mu\right)=\Lambda_{m}^{N}\left(\lambda(N), A_{-k} \mu\right) \\
& =\left(\Lambda_{m}^{N} \delta_{\lambda(N)}\right)\left(A_{-k} \mu\right) \stackrel{N \rightarrow \infty}{\longrightarrow} M_{m}\left(A_{-k} \mu\right)=A_{k} M_{m}(\mu),
\end{aligned}
$$

where we have used $\Lambda_{m}^{N}\left(A_{k} \nu, A_{k} \kappa\right)=\Lambda_{m}^{N}(\nu, \kappa)$, previously stated in the proof of Lemma 6.12.

Let us move on to the second part of the lemma. Let $\mathbf{N}(M)=\nu$; to show $\mathbf{N}\left(A_{k} M\right)=A_{k} \nu$, we need

$$
\begin{aligned}
& \mathcal{P}_{A_{k} M_{m}}\left(x_{1}, \ldots, x_{m}\right)=\Phi^{A_{k} \nu}\left(x_{1} t^{1-m}, \ldots, x_{m-1} t^{-1}, x_{m} ; q, t\right) \\
& \forall\left(x_{1}, \ldots, x_{m}\right) \in \mathbb{T}^{m}, \quad \forall m \in \mathbb{N} .
\end{aligned}
$$

For any $m \in \mathbb{N},\left(x_{1}, \ldots, x_{m}\right) \in \mathbb{T}^{m}$, we have

$$
\begin{aligned}
\mathcal{P}_{A_{k} M_{m}}\left(x_{1}, \ldots, x_{m}\right) & =\sum_{\lambda \in \mathbb{G T}_{m}} A_{k} M_{m}(\lambda) \frac{P_{\lambda}\left(x_{1}, x_{2} t, \ldots, x_{m} t^{m-1}\right)}{P_{\lambda}\left(1, t, t^{2}, \ldots, t^{m-1}\right)} \\
& =\sum_{\lambda \in \mathbb{G T}_{m}} M_{m}\left(A_{-k} \lambda\right) \frac{P_{\lambda}\left(x_{1}, x_{2} t, \ldots, x_{m} t^{m-1}\right)}{P_{\lambda}\left(1, t, t^{2}, \ldots, t^{m-1}\right)} \\
& =\sum_{\lambda \in \mathbb{G T}_{m}} M_{m}(\lambda) \frac{P_{A_{k} \lambda}\left(x_{1}, x_{2} t, \ldots, x_{m} t^{m-1}\right)}{P_{A_{k} \lambda}\left(1, t, t^{2}, \ldots, t^{m-1}\right)} \\
& =\left(x_{1} x_{2} \cdots x_{m}\right)^{k} \cdot \sum_{\lambda \in \mathbb{G}_{m}} M_{m}(\lambda) \frac{P_{\lambda}\left(x_{1}, x_{2} t, \ldots, x_{m} t^{m-1}\right)}{P_{\lambda}\left(1, t, t^{2}, \ldots, t^{m-1}\right)} \\
& =\left(x_{1} x_{2} \cdots x_{m}\right)^{k} \cdot \mathcal{P}_{M_{m}}\left(x_{1}, \ldots, x_{m}\right) \\
& =\left(x_{1} \cdots x_{m}\right)^{k} \cdot \Phi^{\nu}\left(x_{1} t^{1-m}, \ldots, x_{m} ; q, t\right) \\
& =t^{k\left(\begin{array}{c}
m \\
2
\end{array}\right)}\left(\left(x_{1} t^{1-m}\right) \cdots\left(x_{m}\right)\right)^{k} \cdot \Phi^{\nu}\left(x_{1} t^{1-m}, \ldots, x_{m} ; q, t\right) \\
& =\Phi^{A_{k} \nu}\left(x_{1} t^{1-m}, \ldots, x_{m} ; q, t\right),
\end{aligned}
$$

where the last equality follows from Lemma 6.11 . 
Proposition 7.6. The Martin boundary $\Omega_{q, t}^{\mathrm{Martin}}$ of the $(q, t)-G T$ graph is bijective to $\mathcal{N}$ under the map $\mathbf{N}$ defined in (7.8) above.

Proof. Step 1. We prove $\mathbf{N}$ is surjective.

Let $\nu \in \mathcal{N}$ be arbitrary; we want to show it belongs to the range of $\mathbf{N}$. From Lemma 7.5, we may assume $\nu_{1}=0$ without any loss of generality. Consider the sequence $\left\{\lambda(N)=\left(\nu_{N} \geq\right.\right.$ $\left.\left.\nu_{N-1} \geq \cdots \geq \nu_{1}\right)\right\}_{N \geq 1}$ of signatures that stabilizes to $\nu$. Let $m \in \mathbb{N}$ be arbitrary. The first claim is that the limit $\lim _{N \rightarrow \infty} M_{m}^{\lambda(N)}(\lambda)$ exists for any $\lambda \in \mathbb{G T}_{m}$.

By definition (6.4), $M_{m}^{\lambda(N)}(\lambda)=\Lambda_{m}^{N}(\lambda(N), \lambda)=0$ unless $\lambda_{m} \geq \lambda(N)_{N}=\nu_{1}=0$. Thus the previous claim is clear if $\lambda_{m}<\nu_{1}=0$, in which case $\lim _{N \rightarrow \infty} M_{m}^{\lambda(N)}(\lambda)=0$. Assume now $\lambda_{m} \geq \nu_{1}=0$, i.e., $\lambda \in \mathbb{G}_{m}^{+}$.

From Theorem 5.5, we have the uniform limit

$$
\begin{aligned}
\lim _{N \rightarrow \infty} & \frac{P_{\lambda(N)}\left(x_{1} t^{1-m}, \ldots, x_{m-1} t^{-1}, x_{m}, t^{-m}, \ldots, t^{1-N}\right)}{P_{\lambda(N)}\left(1, t^{-1}, t^{-2}, \ldots, t^{1-N}\right)} \\
& =\Phi^{\nu}\left(x_{1} t^{1-m}, \ldots, x_{m-1} t^{-1}, x_{m} ; q, t\right)
\end{aligned}
$$

on $\mathbb{T}^{m}$. Correspondingly, the Fourier coefficients of the normalized Macdonald characters converge to those of $\Phi^{\nu}\left(x_{1} t^{1-m}, \ldots, x_{m-1} t^{-1}, x_{m} ; q, t\right)$. Proposition refprop:coherentsequences and the expansion of Corollary 2.6 give

$$
\begin{aligned}
\frac{P_{\lambda(N)}\left(x_{1} t^{1-m}, \ldots, x_{m-1} t^{-1}, x_{m}, t^{-m}, \ldots, t^{1-N}\right)}{P_{\lambda(N)}\left(1, t^{-1}, t^{-2}, \ldots, t^{1-N}\right)} \\
=\sum_{\mu \in \mathbb{G T}_{m}} M_{m}^{\lambda(N)}(\mu) \frac{P_{\mu}\left(x_{1}, x_{2} t, \ldots, x_{m} t^{m-1}\right)}{P_{\mu}\left(1, t, \ldots, t^{m-1}\right)} \\
=\sum_{\kappa \in \mathbb{G T}_{m}} m_{\kappa}\left(x_{1}, x_{2} t, \ldots, x_{m} t^{m-1}\right) \sum_{\mu \in \mathbb{G T}_{m}} \frac{c_{\mu, \kappa} M_{m}^{\lambda(N)}(\mu)}{P_{\mu}\left(1, t, \ldots, t^{m-1}\right)} .
\end{aligned}
$$

Let $\kappa \in \mathbb{G}_{m}$ be arbitrary, and denote $n(\kappa) \stackrel{\text { def }}{=} \kappa_{2}+2 \kappa_{3}+\cdots+(m-1) \kappa_{m}$. Observe that $x_{1}^{\kappa_{1}} \cdots x_{m}^{\kappa}$ appears only in the monomial symmetric polynomial $m_{\kappa}\left(x_{1}, x_{2} t, \ldots, x_{m} t^{m-1}\right)$ and the corresponding term is $t^{n(\kappa)} x_{1}^{\kappa_{1}} \cdots x_{m}^{\kappa_{m}}$, so (7.10) is essentially the Fourier expansion of the prelimit functions in (7.9). Then we have that, for any $\kappa \in \mathbb{G}_{m}$, the following limit

$$
\lim _{N \rightarrow \infty} t^{n(\kappa)} \sum_{\mu \in \mathbb{G T}_{m}} \frac{c_{\mu, \kappa} M_{m}^{\lambda(N)}(\mu)}{P_{\mu}\left(1, t, \ldots, t^{m-1}\right)}
$$

exists and equals the Fourier coefficient of $x_{1}^{\kappa_{1}} \cdots x_{m}^{\kappa_{m}}$ in the function $\Phi^{\nu}\left(x_{1} t^{m-1}, \ldots, x_{m} ; q, t\right)$. As mentioned before, $M_{m}^{\lambda(N)}(\mu)=0$ unless $\mu_{m} \geq \nu_{1} \geq 0$. Thus we can restrict the sum in (7.11) to $\mu \in \mathbb{G T}_{m}^{+}$. From the same analysis as in Proposition 6.10 , we can obtain that the limit $\lim _{N \rightarrow \infty} M_{m}^{\lambda(N)}(\mu)$ exists for any $\mu \in \mathbb{G}_{m}^{+}$with $|\lambda|=n$; let us denote $M_{m}(\mu) \stackrel{\text { def }}{=} \lim _{N \rightarrow \infty} M_{m}^{\lambda(N)}(\mu)$. Immediately from the definition (and Fatou's lemma) it follows that

$$
\begin{aligned}
& M_{m}(\lambda) \geq 0 \quad \forall \lambda \in \mathbb{G}_{m}, \\
& \sum_{\lambda \in \mathbb{G T}_{m}} M_{m}(\lambda) \leq 1 .
\end{aligned}
$$

Recall that we observed $M_{m}^{\lambda(N)}(\lambda)=0$ if $\lambda \notin \mathbb{G} \mathbb{T}_{m}^{+}$, therefore $M_{m}(\lambda)=0$ if $\lambda \notin \mathbb{G} \mathbb{T}_{m}^{+}$. 
Next consider the following function

$$
F\left(x_{1}, \ldots, x_{m}\right)=\sum_{\mu \in \mathbb{G T}_{m}} M_{m}(\mu) \frac{P_{\mu}\left(x_{1}, x_{2} t, \ldots, x_{m} t^{m-1}\right)}{P_{\mu}\left(1, t, \ldots, t^{m-1}\right)} .
$$

Clearly $F$ defines a function on $\mathbb{T}^{m}$, as it is defined by an absolutely convergent series on the $m$-dimensional torus. Moreover its absolute value is upper bounded by 1 , therefore $F \in$ $L^{\infty}\left(\mathbb{T}^{m}\right) \subset L^{2}\left(\mathbb{T}^{m}\right)$. By the same argument preceding (6.6), one shows, for any $\kappa \in \mathbb{G T}_{m}$, that the Fourier coefficient of $x_{1}^{\kappa_{1}} \cdots x_{m}^{\kappa_{m}}$ in $F$ is

$$
t^{n(\kappa)} \sum_{\mu \in \mathbb{G T}_{m}} \frac{c_{\mu, \kappa} M_{m}(\mu)}{P_{\mu}\left(1, t, \ldots, t^{m-1}\right)} .
$$

Observe that, if $\kappa \in \mathbb{G} \mathbb{T}_{m}$ is fixed, the sums in both (7.11) and (7.12) are finite because $c_{\mu, \kappa} M_{m}(\mu)=0$ unless $\mu \geq \kappa$ and $\mu \in \mathbb{G} \mathbb{T}_{m}^{+}$. Therefore $\lim _{N \rightarrow \infty} M_{m}^{\lambda(N)}(\mu)=M_{m}(\mu)$ for all $\mu \in \mathbb{G}_{m}^{+}$ implies the equality between (7.11) and (7.12). In other words, the following convergence holds

$$
\frac{P_{\lambda(N)}\left(x_{1} t^{1-m}, \ldots, x_{m-1} t^{-1}, x_{m}, t^{-m}, \ldots, t^{1-N}\right)}{P_{\lambda(N)}\left(1, t^{-1}, t^{-2}, \ldots, t^{1-N}\right)} \stackrel{\text { Fourier }}{\longrightarrow} F\left(x_{1}, \ldots, x_{m}\right)
$$

in the sense that all Fourier coefficients of the left side of (7.13) converge to the corresponding Fourier coefficients of $F\left(x_{1}, \ldots, x_{m}\right)$, as $N$ tends to infinity. But we already knew that the Fourier coefficients of the left side of (7.13) converge to the corresponding Fourier coefficients of $\Phi^{\nu}\left(x_{1} t^{1-m}, \ldots, x_{m} ; q, t\right)$. Therefore all the Fourier coefficients of the difference $F\left(x_{1}, \ldots, x_{m}\right)-$ $\Phi^{\nu}\left(x_{1} t^{1-m}, \ldots, x_{m} ; q, t\right)$ of square-integrable functions on $\mathbb{T}^{m}$ must be zero. It follows that $F\left(x_{1}, \ldots, x_{m}\right)=\Phi^{\nu}\left(x_{1} t^{1-m}, \ldots, x_{m} ; q, t\right)$. In particular, the equality holds for $x_{1}=\cdots=x_{m}=1$, resulting in

$$
\begin{aligned}
\sum_{\mu \in \mathbb{G T}_{m}} & M_{m}(\mu)=F(1, \ldots, 1)=\Phi^{\nu}\left(t^{1-m}, t^{2-m}, \ldots, 1 ; q, t\right) \\
= & \left.\lim _{N \rightarrow \infty} \frac{P_{\lambda(N)}\left(x_{1} t^{1-m}, \ldots, x_{m-1} t^{-1}, x_{m}, t^{-m}, \ldots, t^{1-N}\right)}{P_{\lambda(N)}\left(1, t^{-1}, t^{-2}, \ldots, t^{1-N}\right)}\right|_{x_{1}=\ldots=x_{m}=1}=\lim _{N \rightarrow \infty} 1=1 .
\end{aligned}
$$

Moreover $\Phi^{\nu}\left(x_{1} t^{1-m}, \ldots, x_{m} ; q, t\right)=F\left(x_{1}, \ldots, x_{m}\right)$ is the Macdonald generating function of $M_{m}$.

We are almost done. For each $m \in \mathbb{N}$, we have constructed probability measures $M_{m}$ as limits of $M_{m}^{\lambda(N)}$ and shown that $\Phi^{\nu}\left(x_{1} t^{1-m}, \ldots, x_{m} ; q, t\right)$ is the generating function of $M_{m}$. Complete the sequence with $M_{0}=\delta_{\varnothing}$, the delta mass at $\varnothing$. We claim that $\left\{M_{m}\right\}_{m \geq 0}$ is a $(q, t)$-coherent sequence. In fact, since $\left\{M_{m}^{\lambda(N)}\right\}_{m=0,1, \ldots, N}$ is a $(q, t)$-coherent sequence, then

$$
M_{m}^{\lambda(N)}(\mu)=\sum_{\lambda \in \mathbb{G T}_{m+1}} M_{m+1}^{\lambda(N)}(\lambda) \Lambda_{m}^{m+1}(\lambda, \mu)
$$

for any $0 \leq m<N, \mu \in \mathbb{G T}_{m}$. As $N$ goes to infinity, the left side of (7.14) tends to $M_{m}(\mu)$. By an argument similar to that in the proof of Proposition 6.9, one shows that the right side of (7.14) converges to $\sum_{\lambda \in \mathbb{G}_{m+1}} M_{m+1}(\lambda) \Lambda_{m}^{m+1}(\lambda, \mu)$. Indeed, the argument simply relies on the weak convergence $M_{m+1}^{\lambda(N)} \rightarrow M_{m+1}$ and the uniform (on $\lambda$ ) bound $\left|\Lambda_{m}^{m+1}(\lambda, \mu)\right|=\Lambda_{m}^{m+1}(\lambda, \mu) \leq 1$. Therefore the limit of (7.14) as $N \rightarrow \infty$ is

$$
M_{m}(\mu)=\sum_{\lambda \in \mathbb{G T}_{m}} M_{m+1}(\lambda) \Lambda_{m}^{m+1}(\lambda, \mu)
$$


for any $m \in \mathbb{Z}_{\geq 0}, \mu \in \mathbb{G}_{m}$. Thus $\left\{M_{m}\right\}_{m \geq 0}$ is a $(q, t)$-coherent sequence and has an associated probability measure $M$ on $\mathcal{T}$, as given by Proposition 6.5. By the definition of $\mathbf{N}$, we conclude $\mathbf{N}(M)=\nu$.

Step 2. Next we show that $\mathbf{N}$ is injective.

Let $M, M^{\prime} \in \Omega_{q, t}^{\mathrm{Martin}}$ have the same image $\nu$ under the map $\mathbf{N}$. The goal is to prove $M=M^{\prime}$. From Lemma 7.5, we may assume $\nu_{1}=0$ without any loss of generality. Furthermore, we can assume that $M$ is the element of $\Omega_{q, t}^{\mathrm{Martin}}$ such that $\mathbf{N}(M)=\nu$ and that was contructed in the first step.

Let $\left\{M_{m}\right\}_{m \geq 0}$ and $\left\{M_{m}^{\prime}\right\}_{m \geq 0}$ be the $(q, t)$-coherent sequences associated to $M, M^{\prime}$, then

$$
\mathcal{P}_{M_{1}}(x)=\Phi^{\nu}(x ; q, t)=\mathcal{P}_{M_{1}^{\prime}}(x) \quad \forall x \in \mathbb{T}
$$

As it was mentioned in Section 6.3, Macdonald generating functions are uniformly bounded on the torus, in particular, $\mathcal{P}_{\mathfrak{m}} \in L^{\infty}(\mathbb{T}) \subset L^{2}(\mathbb{T})$ for any probability measure $\mathfrak{m}$ on $\mathbb{G}_{1}=\mathbb{Z}$. Write the first equality of (7.15) for $x=e^{i \theta}$ as follows:

$$
\sum_{n \in \mathbb{Z}} M_{1}(n) e^{i n \theta}=\sum_{n \in \mathbb{Z}} M_{1}^{\prime}(n) e^{i n \theta}
$$

Both sums above are expansions of a square integrable function on $\mathbb{T}$ in terms of the basis $\left\{e^{i n \theta}\right\} \subset L^{2}(\mathbb{T})$. Thus the (Fourier) coefficients in both sums must agree, i.e., $M_{1}(n)=M_{1}^{\prime}(n)$ $\forall n \in \mathbb{Z}$, and so $M_{1}=M_{1}^{\prime}$.

We aim to apply a similar argument to show that $M_{m}=M_{m}^{\prime}$ for any $m \in \mathbb{N}$. We will be done once this is proved, as Proposition 6.5 would then show $M=M^{\prime}$. For general $m$, we make use of the fact that $M \in \Omega_{q, t}^{\mathrm{Martin}}$ is the probability measure constructed in step 1: we use that each $M_{m}$ is supported on $\mathbb{G} \mathbb{T}_{m}^{+}$.

As above, the definition of the map $\mathbf{N}$ implies

$$
\begin{aligned}
\mathcal{P}_{M_{m}}\left(x_{1}, \ldots, x_{m}\right) & =\Phi^{\nu}\left(x_{1} t^{1-m}, \ldots, x_{m-1} t^{-1}, x_{m} ; q, t\right) \\
& =\mathcal{P}_{M_{m}^{\prime}}\left(x_{1}, \ldots, x_{m}\right) \quad \forall\left(x_{1}, \ldots, x_{m}\right) \in \mathbb{T}^{m} .
\end{aligned}
$$

The equality of the functions above implies the equality of corresponding Fourier coefficients. It follows that for any $\kappa \in \mathbb{G} \mathbb{T}_{m}$ we have, see (6.6),

$$
\sum_{\mu \in \mathbb{G T}_{m}} \frac{c_{\mu, \kappa} M_{m}(\mu)}{P_{\mu}\left(1, t, \ldots, t^{m-1}\right)}=\sum_{\mu \in \mathbb{G T}_{m}} \frac{c_{\mu, \kappa} M_{m}^{\prime}(\mu)}{P_{\mu}\left(1, t, \ldots, t^{m-1}\right)}
$$

When $\kappa \notin \mathbb{G}_{m}^{+}$(or equivalently $\kappa_{m}<0$ ), we claim that the left side of (7.16) is zero. In fact, for any $\mu \in \mathbb{G T}_{m}$, either $c_{\mu, \kappa}=0$ when $\mu_{m} \geq 0$ or $M_{m}(\mu)=0$ when $\mu_{m}<0$, because $M_{m}$ is supported on $\mathbb{G T}_{m}^{+}$. Then also the right side of (7.16) is zero if $\kappa \notin \mathbb{G T}_{m}^{+}$. By using also the properties of the coefficients $c_{\mu, \kappa}$ stated in Corollary 2.6, we have

$$
0=\sum_{\mu \in \mathbb{G T}_{m}} \frac{c_{\mu, \kappa} M_{m}^{\prime}(\mu)}{P_{\mu}\left(1, t, \ldots, t^{m-1}\right)} \geq \frac{c_{\kappa, \kappa} M_{m}^{\prime}(\kappa)}{P_{\kappa}\left(1, t, \ldots, t^{m-1}\right)}=\frac{M_{m}^{\prime}(\kappa)}{P_{\kappa}\left(1, t, \ldots, t^{m-1}\right)} \geq 0
$$

Then we must have $M_{m}^{\prime}(\kappa)=0$ if $\kappa \notin \mathbb{G} \mathbb{T}_{m}^{+}$, i.e., $M_{m}^{\prime}$ is supported on $\mathbb{G T}_{m}^{+}$. Finally an application of Lemma 6.7 says that if $\mathcal{P}_{M_{m}}=\mathcal{P}_{M_{m}^{\prime}}$ on $\mathbb{T}^{m}$, and $M_{m}, M_{m}^{\prime}$ are both supported on $\mathbb{G T}_{m}^{+}$, implies $M_{m}=M_{m}^{\prime}$, thus finishing the proof. 


\subsection{Characterization of the Martin boundary}

Our next goal is to characterize the topological space $\Omega_{q, t}^{\mathrm{Martin}}$ completely. Recall the map $\mathbf{N}: \Omega_{q, t}^{\text {Martin }} \rightarrow \mathcal{N}$, defined above in (7.8), by letting $\mathbf{N}(M)=\nu$ be the unique element of $\mathcal{N}$ such that

$$
\Phi^{\nu}\left(x_{1} t^{1-m}, \ldots, x_{m-1} t^{-1}, x_{m} ; q, t\right)=\mathcal{P}_{M_{m}}\left(x_{1}, \ldots, x_{m}\right) \quad \forall\left(x_{1}, \ldots, x_{m}\right) \in \mathbb{T}^{m}, \quad \forall m \geq 1 .
$$

Proposition 7.6 shows that $\mathbf{N}$ is a bijection, so the inverse map $\mathbf{N}^{-1}$ is well-defined.

Definition 7.7. For any $\nu \in \mathcal{N}$, we denote $\mathbf{N}^{-1}(\nu)$ by $M^{\nu}$ and the corresponding $(q, t)$-coherent sequence by $\left\{M_{m}^{\nu}\right\}_{m \geq 0}$.

From step 1 of the proof of Proposition 7.6, and Lemma 7.5, we have that for the sequence of signatures $\left\{\lambda(N)=\left(\nu_{N} \geq \cdots \geq \nu_{1}\right)\right\}_{N \geq 1}$ which stabilizes to $\nu$, the following weak convergence holds

$$
\mathbb{P}-\lim _{N \rightarrow \infty} \Lambda_{m}^{N} \delta_{\lambda(N)}=M_{m}^{\nu} \quad \forall m \in \mathbb{N}
$$

i.e.,

$$
\lim _{N \rightarrow \infty} \Lambda_{m}^{N} \delta_{\lambda(N)}(\mu)=\lim _{N \rightarrow \infty} \Lambda_{m}^{N}(\lambda(N), \mu)=M_{m}^{\nu}(\mu) \quad \forall m \in \mathbb{N}, \quad \forall \mu \in \mathbb{G T}_{m} .
$$

In fact, we note the same analysis as in step 1 of the proof of Proposition 7.6 shows that the weak convergence above holds for any sequence of signatures $\{\lambda(N)\}_{N \geq 1}$ stabilizing to $\nu$.

We need the following lemma to prove that $\mathbf{N}$ is a homeomorphism.

\section{Lemma 7.8.}

1. For any $\nu \in \mathcal{N}, m \in \mathbb{N}, M_{m}^{\nu}$ is supported on $\left\{\mu=\left(\mu_{1} \geq \cdots \geq \mu_{m-1} \geq \mu_{m}\right) \in \mathbb{G}_{m}: \mu_{m} \geq\right.$ $\left.\nu_{1}, \mu_{m-1} \geq \nu_{2}, \ldots, \mu_{1} \geq \nu_{m}\right\}$.

2. For any $m \in \mathbb{N}$, there exists $c_{m} \in(0,1)$ such that for any integers $n_{1} \leq n_{2} \leq \cdots \leq n_{m}$ and $\nu \in \mathcal{N}$ with $\nu_{1}=n_{1}, \nu_{2}=n_{2}, \ldots, \nu_{m}=n_{m}$, we have $M_{m}^{\nu}\left(n_{m} \geq \cdots \geq n_{2} \geq n_{1}\right) \geq c_{m}$.

3. Let $m \in \mathbb{N}$ and let $\widetilde{\nu}, \nu \in \mathcal{N}$ be such that $\widetilde{\nu}_{i} \geq \nu_{i} \forall i>m$ and $\widetilde{\nu}_{i}=\nu_{i} \forall 1 \leq i \leq m$, then $M_{m}^{\widetilde{\nu}}\left(\nu_{m} \geq \cdots \geq \nu_{2} \geq \nu_{1}\right) \leq M_{m}^{\nu}\left(\nu_{m} \geq \cdots \geq \nu_{2} \geq \nu_{1}\right)$.

Proof. Let us prove (1). We use the weak convergence of probabilities $\mathbb{P}-\lim _{N \rightarrow \infty} \Lambda_{m}^{N} \delta_{\lambda(N)}=M_{m}^{\nu}$ $\forall m \in \mathbb{N}$, for the sequence of signatures $\left\{\lambda(N)=\left(\nu_{N} \geq \cdots \geq \nu_{2} \geq \nu_{1}\right)\right\}_{N \geq 1}$. Because of the definition (6.4) for the maps $\Lambda_{m}^{N}$, it follows that $\Lambda_{m}^{N} \delta_{\lambda(N)}(\mu)=\Lambda_{m}^{N}(\lambda(N), \mu)=0$, unless $\mu_{m} \geq$ $\lambda(N)_{N}=\nu_{1}, \ldots, \mu_{1} \geq \lambda(N)_{N-m+1}=\nu_{m}$. Thus also $M_{m}^{\nu}(\mu)=0$ unless $\mu_{m} \geq \nu_{1}, \ldots, \mu_{1} \geq \nu_{m}$.

Next we show (2); to get started fix $n_{1} \in \mathbb{N}$ and let us show that $M_{1}^{\nu}\left(n_{1}\right) \geq\left((t ; t)_{\infty}\right)^{\theta}$ for any $\nu \in \mathcal{N}$ with $\nu_{1}=n_{1}$ (so we can set $c_{1}=\left((t ; t)_{\infty}\right)^{\theta}$ ). The weak convergence mentioned above gives $M_{1}^{\nu}\left(n_{1}\right)=\lim _{N \rightarrow \infty} \Lambda_{1}^{N} \delta_{\lambda(N)}\left(n_{1}\right)=\lim _{N \rightarrow \infty} \Lambda_{1}^{N} \delta_{\lambda(N)}\left(\lambda(N)_{N}\right)$, for any $\nu \in \mathcal{N}$ with $\nu_{1}=n_{1}$ and $\{\lambda(N)\}_{N \geq 1}$ as constructed above. The calculations in the proof of Lemma 7.2 show $\Lambda_{1}^{N} \delta_{\lambda(N)}\left(\lambda(N)_{N}\right) \geq\left((t ; t)_{\infty}\right)^{\theta}$ for all $N \in \mathbb{N}$, and thus also $M_{1}^{\nu}\left(n_{1}\right) \geq c_{1} \stackrel{\text { def }}{=}\left((t ; t)_{\infty}\right)^{\theta}>0$, for any $\nu \in \mathcal{N}$ with $\nu_{1}=n_{1}$.

For a general $m \in \mathbb{N}$, we have

$$
M_{m}^{\nu}\left(n_{m} \geq \cdots \geq n_{2} \geq n_{1}\right)=\lim _{N \rightarrow \infty} \Lambda_{m}^{N} \delta_{\lambda(N)}\left(\lambda(N)_{N-m+1} \geq \cdots \geq \lambda(N)_{N}\right),
$$

for $\{\lambda(N)\}_{N \geq 1}$ as constructed above. In the proof of Lemma 7.3, we showed $\Lambda_{m}^{N} \delta_{\lambda(N)}\left(\lambda(N)_{N-m+1}\right.$ $\left.\geq \cdots \geq \lambda(N)_{N}\right) \geq c_{m} \stackrel{\text { def }}{=}\left((t ; t)_{\infty}\right)^{\theta m}$ holds for all $N \geq 1$. Therefore $M_{m}^{\nu}\left(n_{m} \geq \cdots \geq n_{2} \geq n_{1}\right) \geq$ $c_{m}>0$, for any $\nu \in \mathcal{N}$ with $\nu_{1}=n_{1}, \nu_{2}=n_{2}, \ldots, \nu_{m}=n_{m}$. 
Finally we move on to (3). To get started, we prove it for $m=1$, so let $\widetilde{\nu}, \nu \in \mathcal{N}$ be such that $\widetilde{\nu}_{i} \geq \nu_{i} \forall i \geq 2$ and $\widetilde{\nu}_{1}=\nu_{1}$. Consider the following pair of sequences of signatures $\left\{\widetilde{\lambda}(N)=\left(\widetilde{\nu}_{N} \geq \cdots \geq \widetilde{\nu}_{2} \geq \widetilde{\nu}_{1}\right)\right\}_{N \geq 1}$ and $\left\{\lambda(N)=\left(\nu_{N} \geq \cdots \geq \nu_{2} \geq \nu_{1}\right)\right\}_{N \geq 1}$. Then we have the weak limits

$$
\mathbb{P}-\lim _{N \rightarrow \infty} \Lambda_{m}^{N} \delta_{\widetilde{\lambda}(N)}=M_{m}^{\widetilde{\nu}}, \quad \mathbb{P}-\lim _{N \rightarrow \infty} \Lambda_{m}^{N} \delta_{\lambda(N)}=M_{m}^{\nu} \forall m \in \mathbb{N} .
$$

By the calculations in Lemma 7.2, we have

$$
\begin{aligned}
\Lambda_{1}^{N} \delta_{\lambda(N)}\left(\nu_{1}\right) & =\Lambda_{1}^{N} \delta_{\lambda(N)}\left(\lambda(N)_{N}\right) \\
& =\prod_{i=1}^{N-1} \frac{\left(1-t^{N-i}\right)\left(1-q t^{N-i}\right) \cdots\left(1-q^{\theta-1} t^{N-i}\right)}{\left(1-q^{\lambda(N)_{i}-\lambda(N)_{N}} t^{N-i}\right) \cdots\left(1-q^{\lambda(N)_{i}-\lambda(N)_{N}+\theta-1} t^{N-i}\right)} \\
& =\prod_{i=1}^{N-1} \frac{\left(1-t^{N-i}\right)\left(1-q t^{N-i}\right) \cdots\left(1-q^{\theta-1} t^{N-i}\right)}{\left(1-q^{\nu_{N-i+1}-\nu_{1}} t^{N-i}\right) \cdots\left(1-q^{\nu_{N-i+1}-\nu_{1}+\theta-1} t^{N-i}\right)} \\
& \geq \prod_{i=1}^{N-1} \frac{\left(1-t^{N-i}\right)\left(1-q t^{N-i}\right) \cdots\left(1-q^{\theta-1} t^{N-i}\right)}{\left(1-q^{\widetilde{\nu}}\right)} \\
& =\prod_{i=1}^{N-1} \frac{\left(1-t^{N-i}-\widetilde{\nu}_{1} t^{N-i}\right) \cdots\left(1-q q^{\widetilde{\nu_{N-i+1}}-\widetilde{\nu}_{1}+\theta-1} t^{N-i}\right)}{\left(1-q^{\widetilde{\lambda}(N)_{i}-\widetilde{\lambda}(N)_{N}-\theta} t^{N-i+1}\right) \cdots\left(1-q^{\theta-1} t^{N-i}\right)} \\
& =\Lambda_{1}^{N} \delta_{\widetilde{\lambda}(N)}\left(\widetilde{\lambda}(N)_{N}\right)=\Lambda_{1}^{N} \delta_{\widetilde{\lambda}(N)}\left(\nu_{1}\right) .
\end{aligned}
$$

Thus taking into account the limits (7.17) for $m=1$, we deduce $M_{1}^{\nu}\left(\nu_{1}\right) \geq M_{1}^{\widetilde{\nu}}\left(\nu_{1}\right)$.

The proof of the third item for a general $m \in \mathbb{N}$ follows from similar calculations that are used to prove Lemma 7.3. We leave the details to the reader.

Theorem 7.9. The bijective map $\mathbf{N}: \Omega_{q, t}^{\mathrm{Martin}} \rightarrow \mathcal{N}$ is a homeomorphism.

Proof. The first step shows that $\mathbf{N}^{-1}$ is continuous and the second one shows that $\mathbf{N}$ is continuous.

Step 1. Let $\left\{M^{\nu^{(i)}}\right\}_{i \geq 1} \subset \Omega_{q, t}^{\mathrm{Martin}}$ and $\left\{\nu^{(i)}\right\}_{i \geq 1}$ be the corresponding images under the $\operatorname{map} \mathbf{N}$. If $\lim _{i \rightarrow \infty} \nu^{(i)}=\nu \in \mathcal{N}$ pointwise, then we prove the weak limit $\mathbb{P}-\lim _{i \rightarrow \infty} M^{\nu^{(i)}}=M^{\nu}$.

By applying some automorphism $A_{k}$ with large $k$, if necessary, and invoking Lemma 7.5, we can assume $\nu_{1} \geq 0$. Observe that $\lim _{i \rightarrow \infty} \nu_{1}^{(i)}=\nu_{1} \geq 0$ implies $\nu_{1}^{(i)} \geq 0$ for large enough $i$. Thus let us also assume $\nu_{1}^{(i)} \geq 0 \forall i \geq 1$, for simplicity.

We reduce our desired statement to simpler ones. We claim that $\lim _{i \rightarrow \infty} \nu^{(i)}=\nu$ implies

$$
\lim _{i \rightarrow \infty} \Phi^{\nu^{(i)}}\left(x_{1}, \ldots, x_{m} ; q, t\right)=\Phi^{\nu}\left(x_{1}, \ldots, x_{m} ; q, t\right) \quad \forall m \in \mathbb{N}
$$

uniformly on $\mathbb{T}^{m}$.

First let us deduce our desired weak convergence from the limit above. Recall that, due to Theorem 5.5, all functions $\left\{\Phi^{\nu^{(i)}}\left(x_{1}, \ldots, x_{m} ; q, t\right)\right\}_{i \geq 1}$ and $\Phi^{\nu}\left(x_{1}, \ldots, x_{m} ; q, t\right)$ are entire functions and, in particular, they are continuous on the torus $\mathbb{T}^{m}$. So the convergence (7.18) implies the convergence of Fourier coefficients. Thus for any $\kappa \in \mathbb{G T}_{m}$ we obtain, see (6.6),

$$
\lim _{i \rightarrow \infty} \sum_{\mu \in \mathbb{G T}_{m}^{+}} \frac{c_{\mu, \kappa} M_{m}^{\nu^{(i)}}(\mu)}{P_{\mu}\left(1, t, \ldots, t^{m-1}\right)}=\sum_{\mu \in \mathbb{G}_{m}^{+}} \frac{c_{\mu, \kappa} M_{m}^{\nu}(\mu)}{P_{\mu}\left(1, t, \ldots, t^{m-1}\right)}
$$


Note that in both sides of (7.19), the sums have been restricted to $\mathbb{G} \mathbb{T}_{m}^{+}$. In fact, since we are assuming $\nu_{1}^{(i)}, \nu_{1} \geq 0 \forall i \geq 1$, Lemma 7.8(1) implies that all probability measures $\left\{M_{m}^{\nu^{(i)}}\right\}_{i \geq 1}, M_{m}^{\nu}$ are supported on $\mathbb{G}_{m}^{+}$.

From Proposition 6.10, the limit (7.19) yields $\lim _{i \rightarrow \infty} M_{m}^{\nu^{(i)}}(\mu)=M_{m}^{\nu}(\mu) \forall \mu \in \mathbb{G}_{m}^{+}$. But also $M_{m}^{\nu^{(i)}}(\mu)=M_{m}^{\nu}(\mu)=0$ for any $i \geq 1$ and any $\mu \notin \mathbb{G} \mathbb{T}_{m}^{+}$. Therefore $\lim _{i \rightarrow \infty} M_{m}^{\nu^{(i)}}(\mu)=M_{m}^{\nu}(\mu)$ holds for all $\mu \in \mathbb{G T}_{m}$, and so $\mathbb{P}-\lim _{i \rightarrow \infty} M_{m}^{\nu^{(i)}}=M_{m}$. Note that we proved the weak convergence for any $m \in \mathbb{N}$. By Proposition 6.5, we conclude $\mathbb{P}-\lim _{i \rightarrow \infty} M^{\nu^{(i)}}=M$.

We are left with the task of proving (7.18). We show the uniform convergence in a neighborhood of the torus $\mathbb{T}^{m}$. But it suffices to prove the limit on compact subsets of $\mathcal{U}_{m}$. From the definition of $\Phi^{\nu}\left(x_{1}, \ldots, x_{m} ; q, t\right)$ on $\mathcal{U}_{m}$, see (5.8), and observing that $\mathbb{T}^{m} \subset \mathcal{U}_{m}$, it follows that the result holds for any $m \in \mathbb{N}$ provided it holds for $m=1$. We prove (7.18) for $m=1$ on an open neighborhood of $\mathbb{T}$.

Clearly a small enough open neighborhood of $\mathbb{T}$ is a subset of $\mathcal{U}$. By the definition of $\Phi^{\nu}(x ; q, t)$ on $\mathcal{U}$, see (5.2), the desired uniform convergence will hold if we verify

$$
\lim _{i \rightarrow \infty} \int_{\mathcal{C}^{+}} x^{z} \prod_{j=1}^{\infty} \frac{\left(q^{-z+\nu_{j}^{(i)}} t^{j} ; q\right)_{\infty}}{\left(q^{-z+\nu_{j}^{(i)}} t^{j-1} ; q\right)_{\infty}}=\int_{\mathcal{C}^{+}} x^{z} \prod_{j=1}^{\infty} \frac{\left(q^{-z+\nu_{j}} t^{j} ; q\right)_{\infty}}{\left(q^{-z+\nu_{j}} t^{j-1} ; q\right)_{\infty}}
$$

uniformly on compact subsets of $x \in \mathbb{C} \backslash\{0\}$. Note that, since all $\left\{\nu_{1}^{(i)}\right\}_{i \geq 1}, \nu_{1}$, are nonnegative, we can take the same contour $\mathcal{C}^{+}$for both of the integrals in (7.20).

The pointwise convergence of integrands in (7.20) is clear. We still need some uniform estimates for the contribution of the tails of the left side in (7.20). This is similar to the proof of Theorem 5.2.

Let $K \subset \mathbb{C} \backslash\{0\}$ be any compact set. Parametrize the tails of $\mathcal{C}^{+}$as $z=r \pm \frac{\pi \sqrt{-1}}{\ln q}$; for $r$ ranging from some large $R>0$ to $+\infty$, we want to show that the contribution of each of these lines is small. We have

$$
\begin{aligned}
\sup _{x \in K} \mid & x^{z} \cdot \prod_{j=1}^{\infty} \frac{\left(q^{-z+\nu_{j}^{(i)}} t^{j} ; q\right)_{\infty}}{\left(q^{-z+\nu_{j}^{(i)}} t^{j-1} ; q\right)_{\infty}} \mid \\
& \leq \operatorname{const} \times|x|^{r} \times \prod_{j=1}^{\infty} \frac{1}{\left(1+q^{-r+\nu_{j}^{(i)}+\theta(j-1)}\right) \cdots\left(1+q^{-r+\nu_{j}^{(i)}+\theta j-1}\right)} \\
& \leq \text { const } \cdot \frac{|x|^{r}}{q^{-k r} q^{\nu_{1}^{(i)}}+\nu_{2}^{(i)}+\cdots+\nu_{k}^{(i)}}=\text { const } \cdot \frac{\left(|x| q^{k}\right)^{r}}{q^{\nu_{1}^{(i)}+\cdots+\nu_{k}^{(i)}}},
\end{aligned}
$$

for any $z=r \pm \frac{\pi \sqrt{-1}}{\ln q}$, and any $k \in \mathbb{N}$. The constant above depends on $K$ but not on $i$. Choose $k \in \mathbb{N}$ large enough so that $a \stackrel{\text { def }}{=} \sup _{x \in K}|x| \cdot q^{k} \in(0,1)$. Since $\lim _{i \rightarrow \infty} \nu_{j}^{(i)}=\nu_{j}$ for all $j=1,2, \ldots, k$, then $\sup _{i \geq 1} q^{-\nu_{1}^{(i)}-\nu_{2}^{(i)}-\cdots-\nu_{k}^{(i)}} \leq \mathrm{const}<\infty$. Then there exists a constant $c=c_{K}>0$, independent of $i$, such that

$$
\sup _{i \geq 1}\left|\sup _{x \in K}\right| x^{z} \cdot \prod_{j=1}^{\infty} \frac{\left(q^{-z+\nu_{j}^{(i)}} t^{j} ; q\right)_{\infty}}{\left(q^{-z+\nu_{j}^{(i)}} t^{j-1} ; q\right)_{\infty}} \mid \leq c \cdot a^{r}, \quad \text { if } \quad z=r \pm \frac{\pi \sqrt{-1}}{\ln q} .
$$

Since $\int_{R}^{\infty} a^{r} \mathrm{~d} r=-e^{R \ln a} / \ln a \stackrel{R \rightarrow \infty}{\longrightarrow} 0$, we have just shown that the contribution of the tails of $\mathcal{C}^{+}$is uniformly small. We can then apply dominated convergence theorem to conclude (7.20), as desired. 
Step 2. As in the step above, let $\left\{M^{\nu^{(i)}}\right\}_{i>1}$ be a sequence in $\Omega_{q, t}^{\text {Martin }}$, whose images under $\mathbf{N}$ are $\left\{\nu^{(i)}\right\}_{i \geq 1}$. If the weak limit $\mathbb{P}-\lim _{i \rightarrow \infty} M^{\nu^{(i)}}=M$ holds, then we show that $M \in \Omega_{q, t}^{\text {Martin }}$, that there a pointwise limit $\lim _{i \rightarrow \infty} \nu^{(i)}=\nu \in \mathcal{N}$ and moreover $M=M^{\nu}$.

Let $m \in \mathbb{N}$ be arbitrary. Thanks to Proposition 6.5 , the limit $\mathbb{P}-\lim _{i \rightarrow \infty} M^{\nu^{(i)}}=M$ implies $\mathbb{P}-\lim _{i \rightarrow \infty} M_{m}^{\nu^{(i)}}=M_{m}$. By Lemma 7.8(2), there exists $c_{m} \in(0,1)$ such that $M_{m}^{\nu^{(i)}}\left(\nu_{m}^{(i)} \geq\right.$ $\left.\cdots \geq \nu_{1}^{(i)}\right) \geq c_{m}>0$ for all $i \geq 1$. Since $M_{m}$ is a probability measure, there exists $N_{0} \in \mathbb{N}$ large enough such that $M_{m}\left(\left(\lambda_{1}, \ldots, \lambda_{m}\right) \in \mathbb{G}_{m}: N_{0} \geq \lambda_{1}, \ldots, \lambda_{m} \geq-N_{0}\right)>1-\frac{c_{m}}{2}$. Then $\mathbb{P}-\lim _{i \rightarrow \infty} M_{m}^{\nu^{(i)}}=M_{m}$ implies $M_{m}^{\nu^{(i)}}\left(\left(\lambda_{1}, \ldots, \lambda_{m}\right) \in \mathbb{G T}_{m}: N_{0} \geq \lambda_{1}, \ldots, \lambda_{m} \geq-N_{0}\right)>1-c_{m}$ for all $i$ large enough. It follows that $N_{0} \geq \nu_{m}^{(i)}, \ldots, \nu_{1}^{(i)} \geq-N_{0}$ for all $i$ large enough. In particular, the sequence $\left\{\nu_{m}^{(i)}\right\}_{i \geq 1}$ is bounded.

By using the boundedness of the sequences $\left\{\nu_{m}^{(i)}\right\}_{i \geq 1}$ and the "diagonal argument" of Proposition 7.4 , there exists a subsequence $1 \leq i_{1}<i_{2}<i_{3}<\cdots$ such that the limits $\lim _{k \rightarrow \infty} \nu_{m}^{\left(i_{k}\right)}$ exist, for all $m \in \mathbb{N}$. In other words, we have the pointwise limit $\lim _{k \rightarrow \infty} \nu^{\left(i_{k}\right)}=\nu$, for some $\nu \in \mathcal{N}$.

We have the obvious implication

$$
\mathbb{P}-\lim _{i \rightarrow \infty} M^{\nu^{(i)}}=M \Longrightarrow \mathbb{P}-\lim _{k \rightarrow \infty} M^{\nu^{\left(i_{k}\right)}}=M,
$$

But step 1 in this proof shows that $\lim _{k \rightarrow \infty} \nu^{\left(i_{k}\right)}=\nu$ implies $\mathbb{P}-\lim _{k \rightarrow \infty} M^{\nu^{\left(i_{k}\right)}}=M^{\nu}$. By uniqueness of weak limits, we have $M=M^{\nu}$.

We are left to show that $\lim _{i \rightarrow \infty} \nu^{(i)}=\nu$. Above we only showed the existence of a subsequence $\left\{i_{k}\right\}_{k \geq 1}$ such that $\lim _{k \rightarrow \infty} \nu^{\left(i_{k}\right)}=\nu$. Nevertheless the same argument gives us more. In fact, it shows that any subsequence $\left\{\nu^{\left(i_{r}\right)}\right\}_{r \geq 1} \subset\left\{\nu^{(i)}\right\}_{i \geq 1}$ must have a subsubsequence $\left\{\nu^{\left(i_{r(s)}\right)}\right\}_{s \geq 1} \subset$ $\left\{\nu^{\left(i_{r}\right)}\right\}_{r \geq 1}$ such that a pointwise limit $\lim _{s \rightarrow \infty} \nu^{\left(i_{r(s)}\right)}=\nu^{\prime}$ exists. Then $M=M^{\nu^{\prime}}=M^{\nu}$, but since the map $\mathbf{N}$ is bijective, we have $\nu=\nu^{\prime}$. We therefore conclude that the sequence $\left\{\nu^{(i)}\right\}_{i \geq 1}$ itself must converge to $\nu$, finishing the proof.

\subsection{Relation between the Martin boundary and the (minimal) boundary}

The basic relation between the Martin and minimal boundary of the $(q, t)$-GT graph is the following statement, which actually holds in a much greater generality.

Proposition 7.10 (consequence of [37, Theorem 6.1]). The following inclusion holds $\Omega_{q, t} \subseteq$ $\Omega_{q, t}^{\mathrm{Martin}}$. In other words, for any $M \in \Omega_{q, t}$, there exists a sequence $\{\lambda(N)\}_{N \geq 1}, \lambda(N) \in \mathbb{G}_{N}$, such that

$$
\mathbb{P}-\lim _{N \rightarrow \infty} M_{m}^{\lambda(N)}=M_{m} \quad \forall m=0,1,2, \ldots
$$

In many examples, especially in the context of asymptotic representation theory, it is known that the Martin boundary of a branching graph is equal to its minimal boundary, e.g., [21, 37]. In our case, we will also prove that this is the case by following the ideas in [21, 41]. The following statement, which also holds in greater generality, will be useful. 
Proposition 7.11 (consequence of [37, Theorem 9.2]). Let $M^{\prime}$ be any probability measure on $M_{\text {prob }}(\mathcal{T})$. There exists a unique Borel probability measure $\pi$ belonging to $\Omega_{q, t}$ such that

$$
M^{\prime}\left(S_{\phi}\right)=\int_{M \in \Omega_{q, t}} M\left(S_{\phi}\right) \pi(\mathrm{d} M),
$$

for any finite path $\phi=\left(\phi^{(0)} \prec \phi^{(1)} \prec \cdots \prec \phi^{(n)}\right)$ in the $(q, t)-G T$ graph.

\subsection{Characterization of the boundary of the $(q, t)$-Gelfand-Tsetlin graph}

Proof of Theorem 1.3. Let us now prove the items (1), (2) stated in the theorem.

(1) Thanks to Proposition 7.10, we have $\Omega_{q, t} \subseteq \Omega_{q, t}^{\text {Martin }} \subseteq M_{\text {prob }}(\mathcal{T})$. We claim that $\Omega_{q, t}^{\text {Martin }} \subseteq$ $\Omega_{q, t}$. Because of Theorem 7.9, the Martin boundary $\Omega_{q, t}^{\text {Martin }}$ (with its induced topology from $M_{\text {prob }}(\mathcal{T})$ ) is homeomorphic to $\mathcal{N}$, under the map $\mathbf{N}$. The claim would then show that the minimal boundary $\Omega_{q, t}$ of the $(q, t)$-GT graph is equal to the Martin boundary $\Omega_{q, t}^{\mathrm{Martin}}$ and therefore homeomorphic to $\mathcal{N}$, under the map $\mathbf{N}$.

Let us prove the claim above. Let $\nu \in \mathcal{N}$ be arbitrary and let $M^{\nu} \in \Omega_{q, t}^{\mathrm{Martin}}$ be the corresponding element of $\Omega_{q, t}^{\text {Martin }}$. Let i: $\Omega_{q, t} \hookrightarrow \Omega_{q, t}^{\text {Martin }}$ be the natural inclusion, considered as a measurable map. By Proposition 7.11, there exists a unique probability measure $\pi$ on $\Omega_{q, t}$ such that

$$
M^{\nu}=\int_{M \in \Omega_{q, t}} M \pi(\mathrm{d} M)=\int_{M \in \Omega_{q, t}^{\mathrm{Martin}}} M\left(\mathfrak{i}_{*} \pi\right)(\mathrm{d} M) .
$$

Note that $i_{*} \pi$ is a probability measure on $\Omega_{q, t}^{\text {Martin }}$ with $\left(i_{*} \pi\right)\left(\Omega_{q, t}\right)=1$. Let $\widetilde{\pi}$ be the pushforward of $\mathfrak{i}_{*} \pi$ under the homeomorphism $\mathbf{N}: \Omega_{q, t}^{\text {Martin }} \rightarrow \mathcal{N}$, so $\widetilde{\pi}$ is a Borel probability measure on $\mathcal{N}$. Equation (7.21) can be rewritten as

$$
M^{\nu}=\int_{\widetilde{\nu} \in \mathcal{N}} M^{\widetilde{\nu}} \widetilde{\pi}(\mathrm{d} \widetilde{\nu})
$$

We make a subclaim: $\tilde{\pi}$ is the delta mass at $\nu \in \mathcal{N}$. Let us first deduce $\Omega_{q, t}^{\text {Martin }} \subseteq \Omega_{q, t}$ from this latter claim. In fact, if $\tilde{\pi}$ is the delta mass at $\nu \in \mathcal{N}$, then $\mathfrak{i}_{*} \pi$ is the delta mass at $M^{\nu}$. But since we had $\left(i_{*} \pi\right)\left(\Omega_{q, t}\right)=1$, then $M^{\nu} \in \Omega_{q, t}$. Since $M^{\nu}$ was an arbitrary element of $\Omega_{q, t}^{\text {Martin }}$, then we conclude $\Omega_{q, t}^{\mathrm{Martin}} \subseteq \Omega_{q, t}$.

Let us now prove the subclaim that the probability measure $\widetilde{\pi}$ on $\mathcal{N}$ satisfying (7.22) must be the delta mass at $\nu \in \mathcal{N}$. We show first that $\widetilde{\pi}$ is supported on $\{\widetilde{\nu} \in \mathcal{N}: \widetilde{\nu} \geq \nu\} \stackrel{\text { def }}{=}\left\{\widetilde{\nu} \in \mathcal{N}: \widetilde{\nu}_{1} \geq\right.$ $\left.\nu_{1}, \widetilde{\nu}_{2} \geq \nu_{2}, \widetilde{\nu}_{3} \geq \nu_{3}, \ldots\right\}$. Since $\widetilde{\pi}$ is a Borel measure, the opposite would mean the existence of $m \in \mathbb{N}$ and $\kappa_{1} \leq \kappa_{2} \leq \cdots \leq \kappa_{m}$, such that $\kappa_{i}<\nu_{i}$ for some $1 \leq i \leq m$, and

$$
\widetilde{\pi}\left(\left\{\widetilde{\nu} \in \mathcal{N}: \widetilde{\nu}_{1}=\kappa_{1}, \ldots, \widetilde{\nu}_{m}=\kappa_{m}\right\}\right)>0 .
$$

As a consequence of (7.22) we have, for all $m \in \mathbb{N}$,

$$
M_{m}^{\nu}=\int_{\widetilde{\nu} \in \mathcal{N}} M_{m}^{\widetilde{\nu}} \widetilde{\pi}(\mathrm{d} \widetilde{\nu})
$$

We can now apply $(7.23)$ to $\kappa=\left(\kappa_{m} \geq \cdots \geq \kappa_{1}\right) \in \mathbb{G}_{m}$ :

$$
M_{m}^{\nu}(\kappa)=\int_{\widetilde{\nu} \in \mathcal{N}} M_{m}^{\widetilde{\nu}}(\kappa) \widetilde{\pi}(\mathrm{d} \widetilde{\nu}) .
$$

From Lemma 7.8(1), the left-hand side of the equality above vanishes, while Lemma 7.8(2) shows that the right-hand side is at least $c_{m} \cdot \widetilde{\pi}\left(\left\{\widetilde{\nu} \in \mathcal{N}: \widetilde{\nu}_{1}=\kappa_{1}, \ldots, \widetilde{\nu}_{m}=\kappa_{m}\right\}\right)>0$, thus there is a contradiction. 
From the fact that $\widetilde{\pi}$ is supported on $\{\widetilde{\nu} \in \mathcal{N}: \widetilde{\nu} \geq \nu\} \stackrel{\text { def }}{=}\left\{\widetilde{\nu} \in \mathcal{N}: \widetilde{\nu}_{1} \geq \nu_{1}, \widetilde{\nu}_{2} \geq \nu_{2}, \ldots\right\}$, and Lemma 7.8, parts (1) (3), we have that (7.23) evaluated at $\left(\nu_{m} \geq \cdots \geq \nu_{1}\right)$ is

$$
\begin{aligned}
M_{m}^{\nu}\left(\nu_{m} \geq \cdots \geq \nu_{1}\right)= & \int_{\widetilde{\nu} \in \mathcal{N}} M_{m}^{\widetilde{\nu}}\left(\nu_{m} \geq \cdots \geq \nu_{1}\right) \widetilde{\pi}(\mathrm{d} \widetilde{\nu})=\int_{\substack{\widetilde{\nu} \in \mathcal{N} \\
\widetilde{\nu} \geq \nu}} M_{m}^{\widetilde{\nu}}\left(\nu_{m} \geq \cdots \geq \nu_{1}\right) \widetilde{\pi}(\mathrm{d} \widetilde{\nu}) \\
= & \int_{\substack{\widetilde{\nu} \in \mathcal{N}, \widetilde{\nu} \geq \nu \\
\widetilde{\nu}_{i}=\nu_{i} \forall i=1, \ldots, m}} M_{m}^{\widetilde{\nu}}\left(\nu_{m} \geq \cdots \geq \nu_{1}\right) \widetilde{\pi}(\mathrm{d} \widetilde{\nu}) \\
& +\int_{\substack{\widetilde{\nu} \in \mathcal{N}, \widetilde{\nu} \geq \nu \\
\widetilde{\nu}}} M_{m}^{\widetilde{\nu}}\left(\nu_{m} \geq \cdots \geq \nu_{1}\right) \widetilde{\pi}(\mathrm{d} \widetilde{\nu}) \\
= & \int_{\substack{\widetilde{\nu} \text { for some } i \in\{1, \ldots, m\} \\
\widetilde{\nu}_{i}=\nu_{i} \forall i=1, \ldots, m}}^{\substack{\widetilde{\nu} \geq \nu \\
\leq}} M_{m}^{\nu}\left(\nu_{m} \geq \cdots \geq \nu_{1}\right) \cdot \widetilde{\pi}\left(\left\{\widetilde{\nu} \in \mathcal{N}: \widetilde{\nu}_{1}=\nu_{1}, \ldots, \widetilde{\nu}_{m}=\nu_{m}\right\}\right) .
\end{aligned}
$$

Next Lemma $7.8(2)$ says that $M_{m}^{\nu}\left(\nu_{m} \geq \cdots \geq \nu_{1}\right) \geq c_{m}>0$, so we must have $\widetilde{\pi}\left(\left\{\widetilde{\nu} \in \mathcal{N}: \widetilde{\nu}_{1}=\right.\right.$ $\left.\left.\nu_{1}, \ldots, \widetilde{\nu}_{m}=\nu_{m}\right\}\right)=1$. Since this is true for any $m \in \mathbb{N}$, it follows that $\widetilde{\pi}$ must be the delta mass at $\nu$, thus proving our second claim and the full characterization of $\Omega_{q, t}$.

Let us return to the statement of item (1) in Theorem 1.3. By definition of the map $\mathbf{N}$, the relations (1.2) hold. We have already observed that the Macdonald generating function defining $\mathcal{P}_{M_{m}^{\nu}}\left(x_{1}, \ldots, x_{m}\right)$ is absolutely convergent on $\mathbb{T}^{m}$. The last statement that says $M^{\nu}$ is determined by the relations (1.2) follows from the uniqueness statement in Proposition 7.4.

(2) Let $\left\{M_{m}^{\nu}\right\}_{m \geq 0},\left\{M_{m}^{A_{k} \nu}\right\}_{m \geq 0}$, be the $(q, t)$-coherent sequences associated to $M^{\nu}$ and $M^{A_{k} \nu}$, respectively. By Lemma 7.5, we have the first statement $M^{A_{k} \nu}\left(S_{A_{k} \phi}\right)=M^{\nu}\left(S_{\phi}\right)$, for any finite path $\phi$. Next by virtue of Lemma $6.12, M_{m}^{A_{k} \nu}=A_{k} M_{m}^{\nu}$ for all $m \geq 0$. Thus by following the definitions, $M_{m}^{A_{k} \nu}\left(A_{k} \lambda\right)=A_{k} M_{m}^{\nu}\left(A_{k} \lambda\right)=M_{m}^{\nu}\left(A_{-k} A_{k} \lambda\right)=M_{m}^{\nu}(\lambda)$, for any $\lambda \in \mathbb{G T}_{m}$ and $m \in \mathbb{Z}_{\geq 0}$.

\section{A Basics on $q$-analysis}

A good reference for the material on $q$-analysis is [2, Chapter 10]. Assume $|q|<1$ is an arbitrary complex number. Most statements work if $q$ is an indeterminate too.

The $q$-numbers and the $q$-factorial are defined by

$$
\begin{aligned}
& {[n]_{q} \stackrel{\text { def }}{=} \frac{1-q^{n}}{1-q}, \quad n \in \mathbb{N},} \\
& {[n]_{q} ! \stackrel{\text { def }}{=}[n]_{q} \cdots[2]_{q}[1]_{q}, \quad n \in \mathbb{N}, \quad[0]_{q} ! \stackrel{\text { def }}{=} 1 .}
\end{aligned}
$$

It is evident that $[n]_{q} \rightarrow n$ and $[n]_{q} ! \rightarrow n$ !, as $q \rightarrow 1$, for any $n \in \mathbb{Z}_{\geq 0}$. Observe that we can also define $[x]_{q}$ for any $x \in \mathbb{C}$, as before, and it also holds that $[x]_{q} \rightarrow x$ as $q \rightarrow 1$. The $q$-Gamma function is defined by

$$
\Gamma_{q}(z) \stackrel{\text { def }}{=}(1-q)^{1-z} \frac{(q ; q)_{\infty}}{\left(q^{z} ; q\right)_{\infty}}
$$

From the definition, the $q$-functional equation

$$
\Gamma_{q}(z+1)=[z]_{q} \Gamma_{q}(z), \quad z \notin\{\ldots,-2,-1,0\},
$$

is evident. The $q$-Gamma function is a meromorphic function with simple poles at $z=$ $0,-1,-2, \ldots$ and all their shifts by an integral multiple of $2 \pi \sqrt{-1} / \ln q$. The $q$-Gamma function has no zeroes in $\mathbb{C}$. Moreover, we have the following convergence to the Gamma function. 
Theorem A.1 ([2, Corollary 10.3.4]). For any $z \in \mathbb{C} \backslash\{\ldots,-2,-1,0\}$, we have

$$
\lim _{q \rightarrow 1} \Gamma_{q}(z)=\Gamma(z)
$$

Remark A.2. As a consequence of Stieltjes-Vitali theorem, the convergence $\lim _{q \rightarrow 1} \Gamma_{q}(z)=\Gamma(z)$ holds uniformly on compact subsets of $\mathbb{C} \backslash\{\ldots,-2,-1,0\}$.

Other important identities we use in our paper are the q-binomial theorems. To state them, we need to define the $q$-Pochhammer symbols $(x ; q)_{n}$ and $(x ; q)_{\infty}$, for any $x \in \mathbb{C}$ and $n \in \mathbb{Z}_{\geq 0}$ by

$$
(x ; q)_{n} \stackrel{\text { def }}{=} \begin{cases}1 & \text { if } n=0 \\ \prod_{i=1}^{n}\left(1-x q^{i-1}\right) & \text { if } n \geq 1 \\ \prod_{i=1}^{\infty}\left(1-x q^{i-1}\right) & \text { if } n=\infty .\end{cases}
$$

Note that $|q|<1$ implies that the product defining $(x ; q)_{\infty}$ is uniformly convergent for $x \in \mathbb{C}$, and thus $(x ; q)_{\infty}$ is an entire function.

The $q$-binomial formula is the following

Theorem A.3 ([2, Theorem 10.2.1]). For $|z|<1$,

$$
\sum_{n=0}^{\infty} \frac{(a ; q)_{n}}{(q ; q)_{n}} z^{n}=\frac{(a z ; q)_{\infty}}{(z ; q)_{\infty}}
$$

Corollary A.4. For $z \in \mathbb{C}, m \in \mathbb{N}$,

$$
\sum_{n=0}^{M} \frac{\left(q^{-1} ; q^{-1}\right)_{M}}{\left(q^{-1} ; q^{-1}\right)_{n}\left(q^{-1} ; q^{-1}\right)_{M-n}}(-1)^{n} q^{-\left(\begin{array}{c}
n \\
2
\end{array}\right) z^{n}}=\left(z ; q^{-1}\right)_{M} .
$$

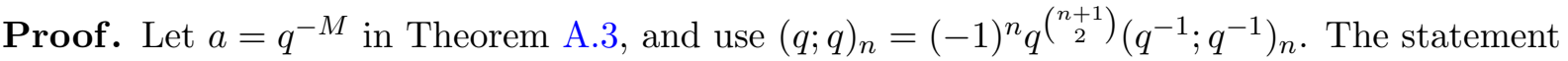
is then proved for any $|z|<1$; therefore it also holds for any $z \in \mathbb{C}$ because both sides are polynomials on $z$.

Another application of the $q$-binomial theorem is the following limit.

Theorem A.5 ([2, Theorem 10.2.4]). For any $a, b \in \mathbb{R}$ such that $b-a \notin \mathbb{Z}$, the following limit

$$
\lim _{q \rightarrow 1} \frac{\left(x q^{a} ; q\right)_{\infty}}{\left(x q^{b} ; q\right)_{\infty}}=(1-x)^{b-a}
$$

holds uniformly on compact subsets of $\{x \in \mathbb{C}:|x| \leq 1, x \neq 1\}$.

Remark A.6. If $b-a \in \mathbb{Z}$, the limit in Theorem A.5 holds uniformly on compact subsets of $\mathbb{C} \backslash\{1\}$.

\section{B Some properties of the rational functions $C_{\tau_{1}, \ldots, \tau_{n}}^{(q, t)}\left(u_{1}, \ldots, u_{n}\right)$}

Lemma B.1. Assume $t=q^{\theta}$, for some $\theta \in \mathbb{N}$. Let $\tau_{1}, \ldots, \tau_{n} \in \mathbb{Z}_{\geq 0}$ and $u_{1}, \ldots, u_{n}$ be $n$ variables, then $C_{\tau_{1}, \ldots, \tau_{n}}^{(q, t)}\left(u_{1}, \ldots, u_{n}\right)=0$ if some of the integers $\tau_{1}, \ldots, \tau_{n}$ is strictly larger than $\theta$. 
Proof. Let us first rewrite the expression for $C_{\tau_{1}, \ldots, \tau_{n}}^{(q, t)}\left(u_{1}, \ldots, u_{n}\right)$, as it was done in [30, Section 6]. Let $v_{i}=q^{\tau_{i}} u_{i}$ for $i=1, \ldots, n$. Also, given $\tau=\left(\tau_{1}, \ldots, \tau_{n}\right)$, let $T=T_{\tau} \stackrel{\text { def }}{=}\left\{k \in\{1,2, \ldots, n\}: \tau_{k} \neq\right.$ $0\}$. Then

$$
\begin{aligned}
C_{\tau}^{(q, t)}\left(u_{1}, \ldots, u_{n}\right)= & \prod_{k \in T} t^{\tau_{k}-1} \frac{(q / t ; q)_{\tau_{k}-1}}{(q ; q)_{\tau_{k}-1}} \frac{\left(q u_{k} ; q\right)_{\tau_{k}}}{\left(q t u_{k} ; q\right)_{\tau_{k}}} \\
& \times \prod_{1 \leq i<j \leq n} \frac{\left(q u_{i} / t u_{j} ; q\right)_{\tau_{i}}}{\left(q u_{i} / u_{j} ; q\right)_{\tau_{i}}} \frac{\left(t u_{j} / q\right)_{\tau_{i}}}{\left(u_{i} / v_{j} ; q\right)_{\tau_{i}}} F_{\tau}(u ; q, t),
\end{aligned}
$$

where

$$
\begin{aligned}
F_{\tau}(u ; q, t) \stackrel{\text { def }}{=} & \sum_{K \subset T}(-1)^{|K|}(1 / t)^{\left(\begin{array}{c}
|K| \\
2
\end{array}\right)} \prod_{j \in T-K} \frac{t-q^{\tau_{j}}}{1-q^{\tau_{j}}} \\
& \times \prod_{\substack{k \in K \\
j \in T-K}} \frac{v_{j}-v_{k} / t}{v_{j}-v_{k}} \prod_{k \in K}\left(\frac{1-t v_{k}}{1-v_{k}} \prod_{\substack{i \in T \\
i \neq k}} \frac{u_{i}-v_{k}}{u_{i}-v_{k} / t}\right) .
\end{aligned}
$$

Due to the factor $\prod_{k \in T}(q / t ; q)_{\tau_{k}-1}$ in (B.1), it follows that $\tau_{k}-\theta \in \mathbb{N}=\{1,2, \ldots\}$, for some $k$, implies $C_{\tau_{1}, \ldots, \tau_{n}}^{(q, t)}\left(u_{1}, \ldots, u_{n}\right)=0$, as desired.

Lemma B.2 ([30, Lemma 6.1]). Assume $t=q$. Let $\tau_{1}, \ldots, \tau_{n} \in \mathbb{Z}_{\geq 0}$ and $u_{1}, \ldots, u_{n}$ be $n$ variables, then $C_{\tau_{1}, \ldots, \tau_{n}}^{(q, t)}\left(u_{1}, \ldots, u_{n}\right)=0$ if some of the integers $\tau_{1}, \ldots, \tau_{n}$ is strictly larger than 1 . If all $\tau_{1}, \ldots, \tau_{n} \in\{0,1\}$, then $C_{\tau_{1}, \ldots, \tau_{n}}^{(q, t)}\left(u_{1}, \ldots, u_{n}\right)$ does not depend on the variables $u_{1}, \ldots, u_{n}$ and

$$
C_{\tau_{1}, \ldots, \tau_{n}}^{(q, t)}=(-1)^{\tau_{1}+\cdots+\tau_{n}}
$$

Lemma B.3. Assume $\theta \in \mathbb{N}$. If we let $a_{n}^{(\theta)}=\frac{C_{n}^{\left(q, q^{\theta}\right)}\left(x_{2}^{-1} x_{1} q^{-\theta}\right)}{\prod_{i=0}^{\theta-1}\left(x_{1}-q^{i} x_{2}\right)}$, for all $0 \leq n \leq \theta$, then these expressions satisfy the relations

$$
\begin{aligned}
& a_{n}^{(\theta)}=\frac{1}{x_{1}-x_{2}}\left(T_{q, x_{2}} a_{n}^{(\theta-1)}-T_{q, x_{1}} a_{n-1}^{(\theta-1)}\right), \quad 1 \leq n \leq \theta-1, \\
& a_{0}^{(\theta)}=\frac{1}{\prod_{i=0}^{\theta-1}\left(x_{1}-q^{i} x_{2}\right)}, \quad a_{\theta}^{(\theta)}=\frac{1}{\prod_{i=0}^{\theta-1}\left(x_{2}-q^{i} x_{1}\right)} .
\end{aligned}
$$

Proof. The expression $C_{n}^{(q, t)}(u)$, for $n \in \mathbb{Z}_{\geq 0}$, is much simpler than the general expression (2.5). It was first found by Jing and Joźefiak in [26] and it reads $C_{n}^{(q, t)}(u)=t^{n} \frac{(1 / t ; q)_{n}}{(q ; q)_{n}} \frac{(u ; q)_{n}}{(q t u ; q)_{n}} \frac{1-q^{2 n} u}{1-u}$. Then, for $0 \leq n \leq \theta$ :

$$
C_{n}^{\left(q, q^{\theta}\right)}\left(x_{1} /\left(q^{\theta} x_{2}\right)\right)=\frac{q^{\theta} x_{2}-q^{2 n} x_{1}}{q^{\theta} x_{2}-x_{1}} \frac{1}{t^{n}} \prod_{i=1}^{n}\left\{\frac{q^{\theta}-q^{i-1}}{1-q^{i}} \frac{q^{\theta} x_{2}-q^{i-1} x_{1}}{x_{2}-q^{i} x_{1}}\right\} .
$$

From (B.2), it is only a matter of tedious computation to check the three identities given in the lemma. 


\section{Acknowledgements}

It is my pleasure to thank Alexei Borodin for his generous sharing of time and ideas. I am equally indebted to Vadim Gorin, for his interest in my work, many helpful discussions and for sharing some of his notes on the $q$-GT graph. This work would not exist without them. I would also like to thank Jiaoyang Huang, for being an excellent sounding board at the beginning stage of this project, Konstantin Matveev for his expert help with the software Mathematica, and Grigori Olshanski for comments in a previous draft of this paper and for asking a question that led to my proof of Theorem 1.3. The suggestions of the referees helped improved this text greatly; many thanks are due to them.

\section{References}

[1] Akemann G., Baik J., Di Francesco P. (Editors), The Oxford handbook of random matrix theory, Oxford University Press, Oxford, 2011.

[2] Andrews G.E., Askey R., Roy R., Special functions, Encyclopedia of Mathematics and its Applications, Vol. 71, Cambridge University Press, Cambridge, 1999.

[3] Borodin A., Bufetov A., Olshanski G., Limit shapes for growing extreme characters of U( $\infty)$, Ann. Appl. Probab. 25 (2015), 2339-2381, arXiv:1311.5697.

[4] Borodin A., Corwin I., Macdonald processes, Probab. Theory Related Fields 158 (2014), 225-400, arXiv:1111.4408.

[5] Borodin A., Corwin I., Sasamoto T., From duality to determinants for q-TASEP and ASEP, Ann. Probab. 42 (2014), 2314-2382, arXiv:1207.5035.

[6] Borodin A., Gorin V., General $\beta$-Jacobi corners process and the Gaussian free field, Comm. Pure Appl. Math. 68 (2015), 1774-1844, arXiv:1305.3627.

[7] Borodin A., Gorin V., Guionnet A., Gaussian asymptotics of discrete $\beta$-ensembles, Publ. Math. Inst. Hautes Études Sci. 125 (2017), 1-78, arXiv:1505.03760.

[8] Borodin A., Olshanski G., Representations of the infinite symmetric group, Cambridge Studies in Advanced Mathematics, Vol. 160, Cambridge University Press, Cambridge, 2017.

[9] Borodin A., Petrov L., Integrable probability: from representation theory to Macdonald processes, Probab. Surv. 11 (2014), 1-58, arXiv:1310.8007.

[10] Bufetov A., Gorin V., Representations of classical Lie groups and quantized free convolution, Geom. Funct. Anal. 25 (2015), 763-814, arXiv:1311.5780.

[11] Bufetov A., Gorin V., Fluctuations of particle systems determined by Schur generating functions, arXiv:1604.01110.

[12] Bufetov A., Knizel A., Asymptotics of random domino tilings of rectangular Aztec diamonds, Ann. Inst. Henri Poincaré Probab. Stat., to appear, arXiv:1604.01491.

[13] Cherednik I., Double affine Hecke algebras and Macdonald's conjectures, Ann. of Math. 141 (1995), 191-216.

[14] Copson E.T., Asymptotic expansions, Cambridge Tracts in Mathematics and Mathematical Physics, Vol. 55, Cambridge University Press, New York, 1965.

[15] Cuenca C., Pieri integral formula and asymptotics of Jack unitary characters, Selecta Math. (N.S.), to appear, arXiv:1704.02430.

[16] Dołęga M., Féray V., On Kerov polynomials for Jack characters, Discrete Math. Theor. Computer Sci. Proc. (2013), 539-550, arXiv:1201.1806.

[17] Dołęga M., Féray V., Gaussian fluctuations of Young diagrams and structure constants of Jack characters, Duke Math. J. 165 (2016), 1193-1282, arXiv:1402.4615.

[18] Dołęga M., Féray V., Śniady P., Jack polynomials and orientability generating series of maps, Sém. Lothar. Combin. 70 (2013), Art. B70j, 50 pages, arXiv:1301.6531.

[19] Edrei A., On the generation function of a doubly infinite, totally positive sequence, Trans. Amer. Math. Soc. 74 (1953), 367-383.

[20] Etingof P.I., Kirillov A.A., Macdonald's polynomials and representations of quantum groups, Math. Res. Lett. 1 (1994), 279-296, hep-th/9312103. 
[21] Gorin V., The $q$-Gelfand-Tsetlin graph, Gibbs measures and $q$-Toeplitz matrices, Adv. Math. 229 (2012), 201-266, arXiv:1011.1769.

[22] Gorin V., From alternating sign matrices to the Gaussian unitary ensemble, Comm. Math. Phys. 332 (2014), 437-447, arXiv:1306.6347.

[23] Gorin V., Olshanski G., A quantization of the harmonic analysis on the infinite-dimensional unitary group, J. Funct. Anal. 270 (2016), 375-418, arXiv:1504.06832.

[24] Gorin V., Panova G., Asymptotics of symmetric polynomials with applications to statistical mechanics and representation theory, Ann. Probab. 43 (2015), 3052-3132, arXiv:1301.0634.

[25] Gorin V., Shkolnikov M., Multilevel Dyson Brownian motions via Jack polynomials, Probab. Theory Related Fields 163 (2015), 413-463, arXiv:1401.5595.

[26] Jing N.H., Józefiak T., A formula for two-row Macdonald functions, Duke Math. J. 67 (1992), $377-385$.

[27] Johansson K., Random matrices and determinantal processes, in Mathematical Statistical Physics, Elsevier B. V., Amsterdam, 2006, 1-55, math-ph/0510038.

[28] Kirillov A.A., Lectures on affine Hecke algebras and Macdonald's conjectures, Bull. Amer. Math. Soc. (N.S.) 34 (1997), 251-292, math.QA/9501219.

[29] Lassalle M., Jack polynomials and free cumulants, Adv. Math. 222 (2009), 2227-2269, arXiv:0802.0448.

[30] Lassalle M., Schlosser M., Inversion of the Pieri formula for Macdonald polynomials, Adv. Math. 202 (2006), 289-325, math.CO/0402127.

[31] Macdonald I.G., A new class of symmetric functions, Sémin. Lothar. Comb. 20 (1988), B20a, 131-171.

[32] Macdonald I.G., Symmetric functions and Hall polynomials, 2nd ed., Oxford Mathematical Monographs, The Clarendon Press, Oxford University Press, New York, 1995.

[33] Noumi M., Macdonald's symmetric polynomials as zonal spherical functions on some quantum homogeneous spaces, Adv. Math. 123 (1996), 16-77, math.QA/9503224.

[34] Okounkov A., Binomial formula for Macdonald polynomials and applications, Math. Res. Lett. 4 (1997), 533-553, q-alg/9608021.

[35] Okounkov A., Infinite wedge and random partitions, Selecta Math. (N.S.) 7 (2001), 57-81, math.RT/9907127.

[36] Okounkov A., Olshanski G., Shifted Jack polynomials, binomial formula, and applications, Math. Res. Lett. 4 (1997), 69-78, q-alg/9608020.

[37] Okounkov A., Olshanski G., Asymptotics of Jack polynomials as the number of variables goes to infinity, Int. Math. Res. Not. 1998 (1998), 641-682, q-alg/9709011.

[38] Okounkov A., Reshetikhin N., Correlation function of Schur process with application to local geometry of a random 3-dimensional Young diagram, J. Amer. Math. Soc. 16 (2003), 581-603, math.CO/0107056.

[39] Okounkov A., Reshetikhin N., Random skew plane partitions and the Pearcey process, Comm. Math. Phys. 269 (2007), 571-609, math.CO/0503508.

[40] Olshanski G., The problem of harmonic analysis on the infinite-dimensional unitary group, J. Funct. Anal. 205 (2003), 464-524, math.RT/0109193.

[41] Olshanski G., Extended Gelfand-Tsetlin graph, its $q$-boundary, and q-B-splines, Funct. Anal. Appl. 50 (2016), 107-130, arXiv:1607.04201.

[42] Olshanski G., Vershik A., Ergodic unitarily invariant measures on the space of infinite Hermitian matrices, in Contemporary mathematical physics, Amer. Math. Soc. Transl. Ser. 2, Vol. 175, Amer. Math. Soc., Providence, RI, 1996, 137-175, math.RT/9601215.

[43] Opdam E.M., Dunkl operators, Bessel functions and the discriminant of a finite Coxeter group, Compositio Math. 85 (1993), 333-373.

[44] Panova G., Lozenge tilings with free boundaries, Lett. Math. Phys. 105 (2015), 1551-1586, arXiv:1408.0417.

[45] Range R.M., Complex analysis: a brief tour into higher dimensions, Amer. Math. Monthly 110 (2003), $89-108$.

[46] Śniady P., Structure coefficients for Jack characters: approximate factorization property, arXiv:1603.04268.

[47] Vershik A.M., Kerov S.V., Characters and factor-representations of the infinite unitary group, Sov. Math. Dokl. 26 (1982), 570-574.

[48] Voiculescu D., Représentations factorielles de type II1 de U( $\infty)$, J. Math. Pures Appl. 55 (1976), 1-20. 